\author{
Universidade de São Paulo \\ Instituto de Física
}

\title{
Setor Eletrofraco Fortemente Acoplado na Escala TeV: Teoria e Fenomenologia no LHC
}

\author{
Eduardo Roberto De Lascio
}

Orientador: Prof. Dr. Gustavo Alberto Burdman

Tese de doutorado apresentada ao Instituto de Física para a obtenção do título de Doutor em Ciências

\section{Banca Examinadora:}

Prof. Dr. Gustavo Alberto Burdman

Prof. Dr. Victor de Oliveira Rivelles

Prof. Dr. Jorge José Leite Noronha Junior

Prof. Dr. Rogério Rosenfeld

Prof. Dr. Adriano Natale
(IF-USP)

(IF-USP)

(IF-USP)

(IFT-Unesp)

(IFT-Unesp) 
FICHA CATALOGRÁFICA

Preparada pelo Serviço de Biblioteca e Informação do Instituto de Física da Universidade de São Paulo

De Lascio, Eduardo Roberto

Setor eletrofraco fortemente acoplado na escala TeV: teoria e fenomenologia no LHC. - São Paulo, 2011.

Tese (Doutorado) - Universidade de São Paulo. Instituto de Física - Depto. de Física de Matemática

Orientador: Prof. Dr. Gustavo Alberto Burdman

Área de Concentração: Física

Unitermos: 1. Física de Partículas; 2. Física de Alta Energia; 3.Física Teórica; 4. Teoria Quântica Relativística; 5. Teoria Quântica de Campo. 


\section{Agradecimentos}

Eu gostaria de agradecer a ajuda a todos os que contribuíram de alguma forma para a realização deste trabalho.

Agradeço a meus pais e minhas irmãs pelo apoio, a minha namorada pelo companheirismo, a meus amigos pelos incentivos e conselhos, a meu orientador por me mostrar diversas ideias e por toda a ajuda, aos professores que por seus conhecimentos e experiência contribuíram com a minha formação.

Agradeço à FAPESP pelo apoio financeiro. 


\section{Resumo}

Esta tese apresenta estudos sobre uma possível extensão do Modelo Padrão, suas características teóricas e fenomenológicas. São estudados aspectos do Modelo Padrão relevantes para a análise do setor eletrofraco. Motivado pela solução do problema da hierarquia, é introduzido um espaço de cinco dimensões, com a quinta dimensão curva e compactificada em orbifold. Neste espaço são analisados campos escalares, espinoriais e vetoriais, exibindo modos excitados, os chamados modos de Kaluza-Klein. É incluída uma quarta geração de férmions, sendo então possível obter um setor eletrofraco fortemente acoplado na escala $\mathrm{TeV}$, com a condensação de quarks. Este mecanismo é consequência de acoplamentos não universais com o primeiro modo de Kaluza-Klein do glúon, acoplamentos mais intensos com a quarta geração de quarks. É feita a determinação dos parâmetros dos quarks no espaço de cinco dimensões, resultando no setor de quarks do Modelo Padrão como limite da teoria a baixas energias. A análise teórica termina com a determinação dos acoplamentos dos quarks com o primeiro modo de Kaluza-Klein do glúon. Valendo-se da propriedade de o primeiro modo excitado do glúon trocar sabor, mesmo sendo uma partícula neutra, um estudo fenomenológico é realizado, mostrando que é possível observar eventos no LHC que indicam haver o setor fortemente acoplado, mediante a aplicação dos cortes descritos neste trabalho. 


\section{Abstract}

This thesis presents studies concerning a possible extension of the Standard Model, its theoretical and phenomenological characteristics. Aspects of the Standard Model that are relevant for the analysis of the electroweak sector are studied. In order to solve the hierarchy problem, a space with five dimensions is introduced, with the fifth dimension curved and compactified in orbifold. Within this space scalar, spinorial and vectorial fields are analysed, showing excited modes, the so-called Kaluza-Klein modes. A fourth generation of fermions is included, which makes it possible to obtain a strongly coupled electroweak sector at the $\mathrm{TeV}$ scale, with quarks condensation. This mechanism is possible due to non-universal couplings with the first KaluzaKlein mode of the gluon, couplings which are stronger with the fourth generation quarks. The quarks parameters in the five-dimensional space are determined, leading to the quark sector of the Standard Model as a low energy limit of the theory. The theoretical analysis is finished by the determination of the quarks couplings with the first Kaluza-Klein mode of the gluon. Using the property of flavor exchange by the gluon, even this being a neutral particle, a phenomenological study is carried out, showing that is possible to observe events at the LHC that indicate the existence of the strongly coupled sector, by means of the use of the cuts described in this work. 


\section{Conteúdo}

1 Introdução 1

2 Modelo Padrão $\quad 7$

2.1 Partículas do Modelo . . . . . . . . . . . . . 7

2.2 Interações . . . . . . . . . . . . . . . . . . 8

2.2.1 Interações Eletrofracas . . . . . . . . . . . 9 9

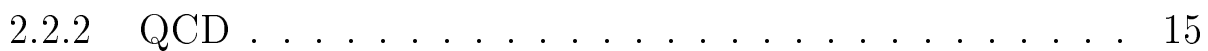

2.3 O Bóson de Higgs . . . . . . . . . . . . . . . . 16

2.4 Extensões do Modelo Padrão . . . . . . . . . . . . . . . . 19

2.4.1 Supersimetria .................. 19

2.4 .2 Technicolor ................... 20

2.4.3 Dimensões Extras . . . . . . . . . . . . . 21

3 Espaço Curvo com Cinco Dimensões 23

3.1 O Espaço .......................... 24

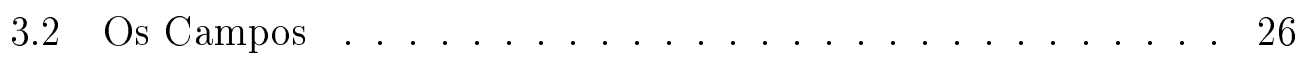

3.2.1 Campo Escalar . . . . . . . . . . . . 26

3.2.2 Campo Espinorial . . . . . . . . . . . . 33

3.2.3 Campo Vetorial . . . . . . . . . . . . . 40 
3.3 Solução ao Problema da Hierarquia . . . . . . . . . . . . . 44

4 Troca de Sabor por Correntes Neutras $\quad 47$

4.1 Extensão do Grupo de Gauge . . . . . . . . . . . . . . . 49

4.2 Modo KK do Glúon . . . . . . . . . . . . . . . . . . . . 52

4.3 Geração de Massas . . . . . . . . . . . . . . . . 54

4.4 Parâmetros dos Quarks . . . . . . . . . . . . 58

4.5 Acoplamentos ..................... 63

$\begin{array}{lll}5 & \text { Fenomenologia } & 67\end{array}$

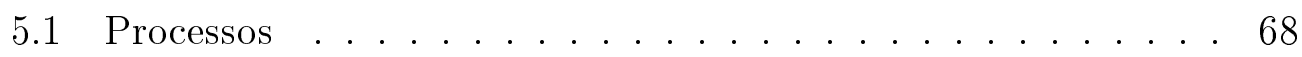

5.1.1 Processo $p p \rightarrow u_{4} \bar{t} \ldots \ldots \ldots . \ldots . \ldots 69$

$5.1 .2 \quad$ Processo $p p \rightarrow d_{4} \bar{b} \ldots \ldots \ldots \ldots 71$

5.2 Deteç̧ão de $p p \rightarrow d_{4} \bar{b} \ldots \ldots \ldots \ldots \ldots$. . . . . . . . . . . .

5.2 .1 Decaimento ................... 72

5.2 .2 Análise e cortes . . . . . . . . . . . 75

5.3 Detecção de $p p \rightarrow u_{4} \bar{t} \ldots \ldots \ldots . \ldots . \ldots 98$

5.3 .1 Decaimento .................... 98

5.3 .2 Análise e cortes . . . . . . . . . . . 100

6 Conclusão 111

$\begin{array}{ll}\text { Bibliografia } & 115\end{array}$ 


\section{Capítulo 1}

\section{Introdução}

Atualmente, a física de partículas elementares encontra-se no limiar de novas descobertas. Com a construção do LHC (Large Hadron Collider) no CERN (Organisation Européenne pour la Recherche Nucléaire) na Suíça, foram abertas as portas para a exploração de fenômenos microscópicos a energias nunca antes alcançadas.

O Modelo Padrão (ver, por exemplo [1, 2]), por um lado, é uma teoria extremamente bem sucedida na descrição de fenômenos experimentais aos quais se tem acesso presentemente. Inclui três das quatro interações ditas fundamentais (não inclui a gravidade) e todas as partículas elementares conhecidas. A teoria tem sido testada exaustivamente ao longo de meio século, ajustando-se com precisão aos mais variados experimentos e testes.

Por outro lado esta teoria é incompleta. O Modelo Padrão depende da introdução de uma partícula adicional, o chamado bóson de Higgs. Esta partícula é um escalar fundamental (em oposição a ser uma consequência da teoria ou uma partícula que se pode decompor) e deve estar presente na teoria para que todas as outras partículas tenham massa. O fato é que, após todos os testes do Modelo Padrão feitos até o presente, o bóson de Higgs não foi detectado.

Até hoje as energias às quais se teve acesso para experimentos em física de partículas chegam a $\sim 2 \mathrm{TeV}$ [1] no TEVATRON (Fermilab), para colisões entre prótons e anti-prótons. Com o LHC, chegar-se-á a $14 \mathrm{TeV}$ de energia no centro de massa, em colisões entre prótons. Desta forma, o Mo- 
delo Padrão será novamente posto à prova, sujeito a testes experimentais a energias inexploradas.

O fato de se explorar energias maiores do que as disponíveis atualmente é suficiente para gerar a expectativa de novas descobertas. Tem sido assim por toda a história da física. A busca pelo bóson de Higgs é a principal motivação para o investimento expressivo feito na construção do LHC e é outro bom motivo para gerar expectativas: caso seja encontrado, deve-se estudar o que está além e verificar se o Modelo Padrão vale até $\sim \mathrm{O}(1 \mathrm{TeV})$; caso não seja encontrado, deve-se reformular a teoria para acomodar este fato. Em ambos os casos deve haver o entendimento teórico dos resultados experimentais e suas consequências.

Há, ainda, limitações sobre a massa do bóson de Higgs, como será visto no capítulo 2. Caso sua massa seja $\gtrsim 600 \mathrm{GeV}$, o Higgs se torna fortemente acoplado [2], deixando de valer a teoria de perturbações e sendo questionável a existência do bóson como partícula elementar.

O fato de ser o bóson de Higgs uma partícula escalar elementar impõe correções quânticas à sua massa (ao quadrado) que divergem quadraticamente com o corte da teoria. Define-se o corte como o limite de energia abaixo do qual a teoria é válida. A massa do Higgs ao quadrado é dada pela soma de duas parcelas: uma proveniente das correções quânticas, proporcional ao corte da teoria ao quadrado $\left(\Delta M_{H}^{2}\right)$; a outra parcela (bare mass) é um parâmetro da lagrangeana $\left(M_{0}^{2}\right)$ :

$$
M_{H}^{2}=M_{0}^{2}+\Delta M_{H}^{2}
$$

Nota-se então que, caso o limite de validade (e portanto o corte da teoria) seja aproximadamente $1 \mathrm{TeV}$, haverá nova física ao alcance do LHC; se o limite de validade do Modelo Padrão estiver muito acima de $1 \mathrm{TeV}$, o parâmetro da lagrangeana também deve estar, de forma que haja um grande cancelamento e reste a massa do Higgs, que é menor do que $1 \mathrm{TeV}$. Ou seja, é necessário um ajuste fino entre o corte da teoria e o parâmetro de massa da lagrangeana. O fato de haver um cancelamento tão grande acabou ganhando o nome de problema da hierarquia e sugere que haja efetivamente nova física na escala $\mathrm{TeV}$. 
Espera-se o corte da teoria na escala da massa de Planck [1]:

$$
M_{P}=1,22089(6) \times 10^{19} \mathrm{GeV}
$$

porque esta é a escala onde os efeitos da gravitação quântica seriam observáveis, sendo necessário um novo modelo que a incluísse. Então resolver o problema da hierarquia equivale, de fato, a entender o porquê do ajuste fino, ou, alternativamente, a encontrar uma explicação para a diferença entre a escala de validade da teoria $\left(M_{P}\right)$ e a escala de massa do bóson de Higgs.

Uma maneira de resolver o problema da hierarquia seria eliminar o escalar fundamental da teoria. A sua função, de gerar massas para todas as partículas, seria realizada por um condensado de férmions [3], que não possuem correções quadraticamente divergentes às suas massas. Para tanto, é necessário que haja acoplamentos fortes que façam um (ou mais) quarks condensarem na escala $\mathrm{TeV}$, enquanto os demais quarks não condensam. Ou seja, o setor eletrofraco deve estar fortemente acoplado, na escala $\mathrm{TeV}$, por meio de interações não universais entre bósons de gauge e férmions.

Tal teoria é realizada por uma modificação do chamado modelo de RandallSundrum [4]. Neste modelo, o espaço-tempo quadridimensional é estendido, ganhando uma dimensão espacial adicional curva. A dimensão extra é também compactificada, de forma que seus efeitos distintivos não sejam observáveis abaixo da escala $\mathrm{TeV}$.

As consequências do modelo aparecem como partículas novas e mais massivas, os modos de Kaluza-Klein, que introduzem novos efeitos. Uma modificação [5] do modelo original de Randall-Sundrum permite que todos os campos do Modelo Padrão, exceto o campo do Higgs, propaguem-se pela quinta dimensão, o que introduz o problema de se obter as formas das funções de onda 5-dimensionais $[5,6,7]$.

As partículas passam a ter novos acoplamentos não-universais, ou seja, férmions, com os mesmos números quânticos, acoplam-se com intensidades distintas aos diversos modos de Kaluza-Klein dos bósons de gauge. Os acoplamentos são agora dependentes das formas das funções de onda 5dimensionais. Os fémions mais massivos se acoplam com intensidade superior aos modos de Kaluza-Klein de bósons.

O bóson de Higgs pode ou não estar presente na teoria. Caso esteja, deve estar localizado próximo a um dos pontos fixos da compactificação. Se 
não estiver, caso haja um condensado que o substitua, o condensado deve possuir massa compatível com a localização próxima ao ponto fixo do Higgs [8]. No caso de haver um condensado, deve estar presente também uma quarta geração de quarks (e de léptons), pois nenhum quark conhecido é massivo o suficiente para condensar na escala $\mathrm{TeV}$.

Sendo mais massivos os quarks da quarta geração, bem como os modos de Kaluza-Klein dos bósons de gauge, compreende-se a ausência de observações experimentais dessas partículas até o presente. Entretanto, vínculos de precisão eletrofracos permitem que os parâmetros de ambos, quarks e bósons, sejam compatíveis com observação no LHC. Por causa de sua localização, os quarks da quarta geração se acoplam muito intensamente ao primeiro modo de Kaluza-Klein do glúon, com acoplamento superior ao da QCD. O modo de Kaluza-Klein do glúon, por sua vez, possui massa $\mathrm{O}(\mathrm{TeV})$, podendo ser produzido no LHC. A seção de choque do decaimento desse modo KK em quarks da quarta geração favorece a observação de processos que envolvam o primeiro modo excitado do glúon e os quarks mais massivos, devendo ser este tipo de processo o primeiro desta classe de modelos a ser observado no LHC.

Inicialmente, poder-se-ia considerar processos nos quais há produção de pares de quarks da quarta geração, a partir do primeiro modo de KaluzaKlein do glúon. Este processo, devido a seu fundo muito expressivo (produção de pares pela QCD), exigiria, para observação do primeiro modo de KaluzaKlein do glúon, luminosidade proibitiva no LHC, devendo ser este método descartado [9].

Devido ao fato de os acoplamentos entre o primeiro modo de KaluzaKlein do glúon e os quarks não serem universais, em conjunto com o fato de haver mistura entre as gerações de quarks no setor eletrofraco, por meio da matriz CKM [2], chega-se a concluir que o primeiro modo de KaluzaKlein do glúon troca sabor. Sendo uma partícula neutra, surge um fato novo e distintivo desta classe de teorias: a troca de sabor por correntes neutras, que em outras teorias seria prejudicial, ocorre a altas energias, sem violar os vínculos de precisão experimentais da física de sabor e fornecendo uma maneira alternativa de se observar seu sinal experimentalmente. Dado que na produção de pares $u_{4} \bar{u}_{4}, d_{4} \bar{d}_{4}$ via QCD não há troca de sabor a nível árvore, devem ser observados, como sinais distintivos desta teoria, os 
processos: $p p \rightarrow u_{4} \bar{t}$ e $p p \rightarrow d_{4} \bar{b}$, onde ocorre a troca de sabor.

A tese trata dessa teoria e de suas consequências experimentais e está dividida em 6 capítulos. Este primeiro serve de introdução. O segundo capítulo contém uma breve revisão do Modelo Padrão [1, 2], com o objetivo de apresentar uma base para outras teorias, na medida em que se aproveitam suas partículas e alguns de seus mecanismos de interação, mas também para servir de vínculo a novas teorias, pois o Modelo Padrão deve ser obtido como limite a baixas energias de teorias que o estendam. No final do capítulo são apresentadas algumas possíveis extensões do Modelo Padrão.

O terceiro capítulo trata da realização do mecanismo de interações nãouniversais fortemente acopladas ao setor eletrofraco, tema desta tese, ou seja, do espaço curvo no Modelo de Randall-Sundrum, bem como da inclusão de campos nesse espaço.

O quarto capítulo introduz o mecanismo de condensação de quarks da quarta geração [8], isto é, os acoplamentos com o primeiro modo de KaluzaKlein do glúon. A consequência deste tipo de interação, que será aproveitada para viabilizar a observação, é a troca de sabor por correntes neutras. Para se tirar conclusões fenomenológicas deste modelo, é necessária a caracterização dos quarks e a inclusão do Modelo Padrão no espaço 5-dimensional curvo. Desta maneira é feita a caracterização dos quarks, ou seja, suas constantes 5-dimensionais e os acoplamentos com o primeiro modo de Kaluza-Klein do glúon são obtidos, para dois modelos representativos da classe de modelos que esta teoria acomoda.

No quinto capítulo, são mostrados resultados de simulações por método de Monte Carlo [10], para processos que trocam sabor, mostrando a viabilidade de observação dos sinais no LHC, após aplicação de adequada estratégia de cortes. O sexto capítulo traz conclusões a respeito da teoria e da fenomenologia do setor eletrofraco fortemente acoplado no LHC, além de perpectivas.

O objetivo desta tese é mostrar a viabilidade da construção de modelos com acoplamentos fortes na escala $\mathrm{TeV}$ que possibilitem a condensação de quarks em substituição ao bóson de Higgs, assim resolvendo o problema da hierarquia, bem como mostrar a viabilidade da observação de seus efeitos no LHC. 


\section{Capítulo 2}

\section{Modelo Padrão}

O Modelo Padrão (MP) [1, 2] é uma teoria de campos quânticos [11] que descreve os fenômenos sub-atômicos com grande precisão e inclui todas as partículas conhecidas. É de extrema importância para a física de partículas, enquanto serve de ponto de partida para desenvolvimentos teóricos.

Pelo princípio da correspondência, qualquer teoria que venha a substituílo deve incluir seus resultados como caso limite. Então, o estudo do Modelo Padrão serve aqui a dois propósitos: ser base para outras teorias, na medida em que se aproveitam suas partículas e mecanismos de interação e explicitar alguns dos vínculos que o MP impõe sobre outras teorias.

\subsection{Partículas do Modelo}

As partículas do Modelo Padrão são os quarks, os léptons e os bósons de gauge. Há ainda no MP uma partícula, o bóson de Higgs, que foi inserida na teoria para fornecer um mecanismo de obtenção de massas para todas as partículas massivas, mas que até hoje não foi detectada experimentalmente.

Os férmions são organizados em gerações. São conhecidas três gerações no Modelo Padrão, atualmente, apesar de não haver impedimento teórico para que este número seja maior. Cada geração é composta por dois quarks e dois léptons. Essas partículas, para efeito de transformações de gauge, são decompostas em partículas de mão esquerda e de mão direita, sendo que os 
quarks e léptons de mão esquerda, de cada geração, formam dubletos por $S U(2)_{L}$ e os de mão direita são singletos.

No Modelo Padrão os léptons são, da primeira para a terceira geração, elétron e neutrino do elétron, múon e neutrino do múon, tau e neutrino do tau. No MP, os neutrinos não possuem massa, mas caso se confirme experimentalmente as massas dos neutrinos, estas podem ser incluídas no modelo. Os léptons carregados possuem as massas [1]:

$m_{e}=0,51 \mathrm{MeV}, m_{\mu}=105,7 \mathrm{MeV}$ e $m_{\tau}=1777 \mathrm{MeV}$. Nota-se a grande disparidade entre as massas dos léptons, que diferem por 3 ordens de grandeza.

Os quarks, organizados na três gerações, são: up e down, da primeira geração; charm e strange, da segunda; top e bottom, da terceira. As massas são [1] $m_{u}=1,5-3,3 \mathrm{MeV} ; m_{d}=3,5-6,0 \mathrm{MeV} ; m_{c}=1,270 \mathrm{GeV}$, $m_{s}=104 \mathrm{MeV} ; m_{b}=4,200 \mathrm{GeV}$ e $m_{t}=171,200 \mathrm{GeV}$. Nota-se também que há disparidade entre as massas das partículas. O problema da disparidade entre as massas das partículas do Modelo Padrão é conhecido como problema da hierarquia das massas, pois não há explicação conhecida para esta grande variação entre as massas dos férmions mais leves e as massas dos mais pesados.

\subsection{Interações}

O Modelo Padrão inclui três interações, as interações eletromagnéticas, as interações fracas e as fortes. Estas interações são representadas por termos incluídos na densidade de lagrangeana da teoria que respeitam uma certa transformação de gauge, associando um grupo de gauge a uma dada interação.

O grupo de gauge do MP é dado por $S U(3)_{c} \times S U(2)_{L} \times U(1)_{Y}$. O grupo $S U(3)_{c}$ é o grupo da cromodinâmica quântica (QCD), enquanto $S U(2)_{L} \times$ $U(1)_{Y}$ representa o grupo das interações eletrofracas. 


\subsubsection{Interações Eletrofracas}

Todos os férmions elementares conhecidos sofrem interações eletrofracas. Estas respeitam o grupo de gauge $S U(2)_{L} \times U(1)_{Y}$, onde o grupo $S U(2)_{L}$ governa as transformações de isospin fraco e o índice indica que apenas os estados de mão esquerda se transformam de maneira não trivial e $U(1)_{Y}$ é o grupo das transformações de gauge de hipercarga fraca.

A lagrangeana invariante por $S U(2)_{L} \times U(1)_{Y}$ se decompõe em:

$$
\mathcal{L}_{e f}=\mathcal{L}_{G}+\mathcal{L}_{F}+\mathcal{L}_{H}
$$

A lagrangeana de gauge é:

$$
\mathcal{L}_{G}=-\frac{1}{4} F_{i}^{\mu \nu} F_{\mu \nu}^{i}-\frac{1}{4} B^{\mu \nu} B_{\mu \nu}
$$

onde:

$$
F_{\mu \nu}^{i}=\partial_{\mu} W_{\nu}^{i}-\partial_{\nu} W_{\mu}^{i}-g_{2} \epsilon^{i j k} W_{\mu}^{j} W_{\nu}^{k}
$$

é o tensor de campo de $S U(2)_{L}$, sendo $i=1,2,3$ o índice que identifica o gerador de $S U(2)_{L}$ ao qual é associado o campo $W_{\mu}^{i}$ (vide eq. 2.8) e:

$$
B_{\mu \nu}=\partial_{\mu} B_{\nu}-\partial_{\nu} B_{\mu}
$$

é o campo de $U(1)_{Y}$.

Os férmions devem respeitar a equação de Dirac:

$$
\left(i \gamma_{\mu} \partial^{\mu}-m\right) \psi=0
$$

onde o campo $\psi$ representa quarks ou léptons. Como o grupo $S U(2)_{L}$ atua apenas sobre os componentes de mão esquerda (dubletos) de cada campo fermiônico, segue à decomposição dos campos dos férmions:

$$
\psi_{L, R}=\frac{1}{2}\left(1 \mp \gamma^{5}\right) \psi
$$

a lagrangeana fermiônica:

$$
\mathcal{L}_{F}=\sum_{\psi_{L}} \bar{\psi}_{L} i \gamma^{\mu} D_{\mu} \psi_{L}+\sum_{\psi_{R}} \bar{\psi}_{R} i \gamma^{\mu} D_{\mu} \psi_{R}
$$


com as somas sobre os dubletos $\psi_{L}$ e sobre os singletos $\psi_{R}{ }^{1}$. Para os últimos:

$$
D_{\mu} \psi_{R}=\left(\partial_{\mu}+i \frac{g_{1}}{2} Y_{w} B_{\mu}\right) \psi_{R}
$$

e para os primeiros:

$$
D_{\mu} \psi_{L}=\left(\mathbf{I}\left(\partial_{\mu}+i \frac{g_{1}}{2} Y_{w} B_{\mu}\right)+i g_{2} \frac{\vec{\tau}}{2} \cdot \vec{W}_{\mu}\right) \psi_{L}
$$

As matrizes $\tau$ são as matrizes de Pauli, geradores de $S U(2)$ na representação fundamental e $Y_{w}$ é a hipercarga fraca, definida por:

$$
Y_{w}=2\left(Q-T_{w 3}\right),
$$

sendo $Q$ a carga elétrica e $T_{w 3}$ o segundo componente do isospin fermiônico fraco, que para os componentes do tipo up dos dubletos (de $S U(2)_{L}$ ) de quarks vale $1 / 2$ e para os do tipo down vale $-1 / 2$. Para os singletos vale zero. $^{2}$

Finalmente, introduzindo um dubleto complexo:

$$
\Phi=\left(\begin{array}{c}
\varphi^{+} \\
\varphi^{0}
\end{array}\right)
$$

de campos de Higgs de spin zero, são obtidos os componentes de:

$$
\begin{gathered}
\mathcal{L}_{H}=\mathcal{L}_{H G}+\mathcal{L}_{H F}, \\
\mathcal{L}_{H G}=\left(D^{\mu} \boldsymbol{\Phi}\right)^{*} D_{\mu} \boldsymbol{\Phi}-V(\boldsymbol{\Phi}),
\end{gathered}
$$

onde

$$
D_{\mu} \boldsymbol{\Phi}=\left(\mathbf{I}\left(\partial_{\mu}+i \frac{g_{1}}{2} B_{\mu}\right)+i g_{2} \frac{\vec{\tau}}{2} \cdot \vec{W}_{\mu}\right) \mathbf{\Phi}
$$

e

$$
V(\boldsymbol{\Phi})=-\mu^{2} \boldsymbol{\Phi}^{\dagger} \boldsymbol{\Phi}+\lambda\left(\boldsymbol{\Phi}^{\dagger} \boldsymbol{\Phi}\right)^{2}
$$

é a auto-interação do Higgs, com $\lambda$ e $\mu^{2}$ positivos.

\footnotetext{
${ }^{1}$ Nota-se a ausência de termos de massa para os férmions. Ocorre que estes termos de massa são proibidos pois não respeitam a simetria de gauge $S U(2)_{L} \times U(1)_{Y}$. O mesmo ocorre com os bósons de gauge, como pode ser observado na equação 2.2. As massas serão introduzidas pelo mecanismo de Higgs, como será visto adiante.

${ }^{2}$ As definições acima são feitas para que se respeite a invariância de gauge e para definir a normalização do acoplamento $g_{1}$ de $U(1)_{Y}$. No caso do bóson de Higgs, como pode ser notado a seguir, $Y_{w}=1$.
} 
Quanto à lagrangeana de interação Higgs-férmion, considerando-se inicialmente apenas uma geração de férmions, por simplicidade notacional, segue que:

$$
\mathcal{L}_{H F}=-f_{u} \bar{q}_{L} \tilde{\boldsymbol{\Phi}} u_{R}-f_{d} \bar{q}_{L} \boldsymbol{\Phi} d_{R}-f_{e} \bar{l}_{L} \boldsymbol{\Phi} e_{R}+\text { h.c. }
$$

onde as constantes $f_{u}, f_{d}$ e $f_{e}$ são os acoplamentos de Yukawa, relacionados às massas dos férmions e:

$$
\tilde{\boldsymbol{\Phi}}=i \tau_{2} \boldsymbol{\Phi}^{*}
$$

As massas dos férmions surgem com a quebra espontânea de simetria. A minimização de $V\left(\boldsymbol{\Phi}^{\dagger} \boldsymbol{\Phi}\right)$ leva a:

$$
\left(-\mu^{2}+2 \lambda \boldsymbol{\Phi}^{\dagger} \boldsymbol{\Phi}\right)=0
$$

que é interpretada em termos do valor esperado de vácuo de $\boldsymbol{\Phi}$. A equação admite como solução:

$$
\left\langle\boldsymbol{\Phi}^{\dagger} \boldsymbol{\Phi}\right\rangle_{0}=\frac{v^{2}}{2}
$$

com:

$$
v=\sqrt{\frac{\mu^{2}}{\lambda}}
$$

Experimentalmente determina-se o valor [2]: $v=246,221(2)$. Uma configuração que satisfaz a equação 2.18 é:

$$
\langle\boldsymbol{\Phi}\rangle_{0}=\left(\begin{array}{c}
0 \\
v / \sqrt{2}
\end{array}\right)
$$

que, não sendo a única, implica na degenerescência do vácuo. Quando o vácuo é degenerado, deve ser feita uma escolha para o valor esperado de vácuo (VEV) da teoria (por exemplo a equação 2.20) e assim o vácuo deixa de respeitar o grupo de simetria da interação em questão, neste caso é dito que a simetria foi espontaneamente quebrada.

Com a expansão do campo de Higgs em torno do valor esperado de vácuo da equação 2.20, os termos de massa na lagrangeana, decorrentes de 2.15, tornam-se aparentes:

$$
\begin{array}{r}
\mathcal{L}_{m}=-\frac{v}{\sqrt{2}}\left(f_{u} \bar{u} u+f_{d} \bar{d} d+f_{e} \bar{e} e\right)+\left(\frac{v g_{2}}{2}\right)^{2} W_{\mu}^{+} W_{-}^{\mu}+ \\
+\frac{v^{2}}{8}\left(W_{\mu}^{3}, B_{\mu}\right)\left(\begin{array}{cc}
g_{2}^{2} & -g_{1} g_{2} \\
-g_{1} g_{2} & g_{1}^{2}
\end{array}\right)\left(\begin{array}{c}
W_{3}^{\mu} \\
B^{\mu}
\end{array}\right) .
\end{array}
$$


Nota-se que as massas dos férmions são dadas por:

$$
m_{\alpha}=\frac{v}{\sqrt{2}} f_{\alpha}
$$

com $\alpha=u, d, e, \ldots$, sendo que os bósons carregados são dados pelas combinações:

$$
W_{\mu}^{ \pm}=\sqrt{\frac{1}{2}}\left(W_{\mu}^{1} \mp W_{\mu}^{2}\right),
$$

que possuem massa $M_{W}=\frac{v}{2} g_{2}$. Nota-se também que os bósons neutros sofrem mistura, sendo que a diagonalização ocorre na base de estados:

$$
\begin{aligned}
& Z_{\mu}=\cos \theta_{w} W_{\mu}^{3}-\sin \theta_{w} B_{\mu}, \\
& A_{\mu}=\sin \theta_{w} W_{\mu}^{3}+\cos \theta_{w} B_{\mu},
\end{aligned}
$$

levando ao fóton e ao bóson $\mathrm{Z}$, com massas zero e $M_{Z}=\frac{v}{2} \sqrt{g_{1}^{2}+g_{2}^{2}}$, respectivamente. Nestas equações vale:

$$
\tan \left(\theta_{w}\right)=\frac{g_{1}}{g_{2}}
$$

Até o momento a discussão foi baseada em uma geração de férmions. Para sua extensão a mais gerações, é necessário generalizar a lagrangeana de interação Higgs-férmion:

$$
-\mathcal{L}_{H F}=f_{u}^{\alpha \beta} \bar{Q}_{L, \alpha}^{\prime} \tilde{\boldsymbol{\Phi}} U_{R, \beta}^{\prime}+f_{d}^{\alpha \beta} \bar{Q}_{L, \alpha}^{\prime} \boldsymbol{\Phi} D_{R, \beta}^{\prime}+f_{e}^{\alpha \beta} \bar{l}_{L, \alpha}^{\prime} \boldsymbol{\Phi} E_{R, \beta}^{\prime}+\text { h.c. }
$$

sendo que os índices $\alpha$ e $\beta$ representam a geração e a sua repetição implica em soma, a notação adotada significa:

$$
\begin{gathered}
\vec{U}^{\prime}=\left(u^{\prime}, c^{\prime}, t^{\prime}, . .\right)^{T} \\
\vec{D}^{\prime}=\left(d^{\prime}, s^{\prime}, b^{\prime}, \ldots\right)^{T} \\
\vec{E}^{\prime}=\left(e^{\prime}, \mu^{\prime}, \tau^{\prime}, \ldots\right)^{T} \\
\vec{Q}^{\prime}=\left(\left(\begin{array}{c}
u^{\prime} \\
d^{\prime}
\end{array}\right),\left(\begin{array}{c}
c^{\prime} \\
s^{\prime}
\end{array}\right),\left(\begin{array}{c}
t^{\prime} \\
b^{\prime}
\end{array}\right), \ldots\right)^{T} \\
\overrightarrow{l^{\prime}}=\left(\left(\begin{array}{c}
\nu_{e}^{\prime} \\
e^{\prime}
\end{array}\right),\left(\begin{array}{c}
\nu_{\mu}^{\prime} \\
\mu^{\prime}
\end{array}\right),\left(\begin{array}{c}
\nu_{\tau}^{\prime} \\
\tau^{\prime}
\end{array}\right), \ldots\right)^{T}
\end{gathered}
$$


e as linhas sobre campos fermiônicos indicam que estes estados que aparecem originalmente na lagrangeana invariante de gauge não são, em geral, os autoestados de massa. Nota-se também que os coeficientes $f^{\alpha \beta} \operatorname{com} \alpha \neq \beta$ não precisam ser nulos, levando a matrizes de massa que, em geral, não serão diagonais.

As matrizes de massa, que seguem a quebra espontânea de simetria:

$$
\mathbf{m}_{\alpha}^{\prime}=\frac{v}{\sqrt{2}} \mathbf{f}_{\alpha}
$$

com $(\alpha=u, d, e)$, não são diagonais na base de interação (a base onde se escrevem os campos, que respeitam as simetrias de gauge). Assim são introduzidas matrizes n x n unitárias (n é o número de gerações), denotadas por $\mathrm{S}$, de forma que a lagrangeana de massa se torna:

$$
\begin{array}{r}
-\mathcal{L}_{F, m}=\bar{U}_{L}^{\prime} \mathbf{S}_{L}^{u} \mathbf{S}_{L}^{u \dagger} \mathbf{m}_{u}^{\prime} \mathbf{S}_{R}^{u} \mathbf{S}_{R}^{u \dagger} U_{R}^{\prime}+\bar{D}_{L}^{\prime} \mathbf{S}_{L}^{d} \mathbf{S}_{L}^{d \dagger} \mathbf{m}_{d}^{\prime} \mathbf{S}_{R}^{d} \mathbf{S}_{R}^{d \dagger} D_{R}^{\prime}+ \\
+\bar{E}_{L}^{\prime} \mathbf{S}_{L}^{e} \mathbf{S}_{L}^{e \dagger} \mathbf{m}_{e}^{\prime} \mathbf{S}_{R}^{e} \mathbf{S}_{R}^{e \dagger} E_{R}^{\prime}+h . c .= \\
=\bar{U} \mathbf{m}_{u} U+\bar{D} \mathbf{m}_{d} D+\bar{E} \mathbf{m}_{e} E .
\end{array}
$$

As matrizes $\mathbf{S}$ relacionam as bases de gauge e de massa, resultando, por exemplo: $U_{L}^{\prime}=\mathbf{S}_{L}^{u} U_{L}$.

Das equações (2.7) e (2.8) os acoplamentos entre os bósons de gauge e os quarks podem ser postos na forma:

$$
\begin{array}{r}
\mathcal{L}_{\text {inter }}=\frac{-g_{2}}{\sqrt{8}}\left(W_{\mu}^{+} \mathrm{J}_{c a r}^{\mu}+W_{\mu}^{-} \mathrm{J}_{c a r}^{\mu \dagger}\right)-g_{1} \cos \theta_{w} A_{\mu} \mathrm{J}_{\text {em }}^{\mu}- \\
-\frac{g_{2}}{2 \cos \theta_{w}} Z^{\mu}\left[\bar{U}_{L}^{\prime}\left(g_{v}^{(u)} \gamma_{\mu}-g_{a}^{(u)} \gamma_{\mu} \gamma_{5}\right) U_{L}^{\prime}+\bar{D}_{L}^{\prime}\left(g_{v}^{(d)} \gamma_{\mu}-g_{a}^{(d)} \gamma_{\mu} \gamma_{5}\right) D_{L}^{\prime}\right]
\end{array}
$$

As constantes do acoplamento com o bóson $Z$ são:

$$
\begin{gathered}
g_{v}^{(f)}=T_{w 3}^{(f)}-2 \operatorname{sen}^{2} \theta_{w} Q^{(f)}, \\
g_{a}^{(f)}=T_{w 3}^{(f)} .
\end{gathered}
$$

Do termo entre colchetes, que é a corrente neutra fraca para os quarks, segue que, usando também a unitariedade das matrizes $\mathbf{S}$, a corrente neutra não troca sabor, ou seja:

$$
\begin{aligned}
& {\left[\bar{U}_{L}^{\prime}\left(g_{v}^{(u)} \gamma_{\mu}-g_{a}^{(u)} \gamma_{\mu} \gamma_{5}\right) U_{L}^{\prime}+\bar{D}_{L}^{\prime}\left(g_{v}^{(d)} \gamma_{\mu}-g_{a}^{(d)} \gamma_{\mu} \gamma_{5}\right) D_{L}^{\prime}\right]=} \\
& =\left[\bar{U}_{L}\left(g_{v}^{(u)} \gamma_{\mu}-g_{a}^{(u)} \gamma_{\mu} \gamma_{5}\right) U_{L}+\bar{D}_{L}\left(g_{v}^{(d)} \gamma_{\mu}-g_{a}^{(d)} \gamma_{\mu} \gamma_{5}\right) D_{L}\right]
\end{aligned}
$$


sendo evidente que os termos entre parênteses não misturam índices de sabor. A corrente eletromagnética dos quarks tipo up é:

$$
\begin{array}{r}
\mathrm{J}_{e m}^{\mu}=\frac{2}{3} \bar{U}_{\alpha}^{\prime} \gamma^{\mu} U_{\alpha}^{\prime}=\frac{2}{3} \bar{U}_{\alpha L}^{\prime} \gamma^{\mu} U_{\alpha L}^{\prime}+\frac{2}{3} \bar{U}_{\alpha R}^{\prime} \gamma^{\mu} U_{\alpha R}^{\prime}= \\
=\frac{2}{3}\left(\bar{U}_{L} \mathbf{S}_{L}^{u \dagger}\right)_{\alpha} \gamma^{\mu}\left(\mathbf{S}_{L}^{u} U_{L}\right)_{\alpha}+\frac{2}{3}\left(\bar{U}_{R} \mathbf{S}_{R}^{u \dagger}\right)_{\alpha} \gamma^{\mu}\left(\mathbf{S}_{R}^{u} U_{R}\right)_{\alpha}= \\
=\frac{2}{3} \bar{U}_{\alpha L}^{\prime} \gamma^{\mu} U_{\alpha L}^{\prime}+\frac{2}{3} \bar{U}_{\alpha R}^{\prime} \gamma^{\mu} U_{\alpha R}^{\prime}=\frac{2}{3} \bar{U}_{\alpha} \gamma^{\mu} U_{\alpha},
\end{array}
$$

onde fica claro que a corrente eletromagnética também não troca sabor.

Nota-se, pela verificação direta das equações 2.35 e (particularmente) das 2.38, 2.39, que não há correntes neutras que trocam sabor (FCNC) no Modelo Padrão ${ }^{3}$, a mistura de férmions decorrendo do acoplamento com os bósons carregados. A ausência de FCNC decorre do fato de todas as gerações se acoplarem aos bósons de gauge com acoplamentos idênticos.

A corrente carregada de quarks, que se acopla aos bósons $W^{ \pm}$, é dada por:

$$
\mathrm{J}_{c a r}^{\mu}=2 \bar{U}_{L, \alpha}^{\prime} \gamma^{\mu} D_{L, \alpha}^{\prime}=2 \bar{U}_{L, \alpha} \gamma^{\mu} D_{L, \alpha}^{\prime \prime},
$$

onde:

$$
D_{L, \alpha}^{\prime \prime}=\mathbf{V}_{\alpha \beta} D_{L, \beta}
$$

e:

$$
\mathbf{V}=\mathbf{S}_{L}^{u \dagger} \mathbf{S}_{L}^{d}
$$

A matriz unitária $\mathbf{V}$ é a matriz de mistura de quarks, pois não é diagonal. Para três gerações é conhecida como matriz de Cabibbo-Kobayashi-Maskawa (CKM). Vale ressaltar, para o uso no modelo com quatro gerações, que esta matriz pode ser expressa [2], para n gerações, em termos de $n(n-1) / 2$ ângulos (tais qual o ângulo de Cabibbo para $\mathrm{n}=2$ ) e $n(n+1) / 2$ fases, das quais podem ser eliminadas $2 n-1$ por mudança de fase dos quarks.

\footnotetext{
${ }^{3}$ Afirmação válida também para a QCD, como poderá ser verificado a seguir, uma vez que seus termos também são diagonais nos índices de sabor.
} 


\subsubsection{QCD}

O Modelo Padrão inclui ainda a cromodinâmica quântica (QCD), que representa as interações fortes. Sua lagrangeana é:

$$
\mathcal{L}=\sum_{\alpha} \bar{\psi}_{j}^{(\alpha)}\left(i \gamma^{\mu} D_{\mu j k}-m^{(\alpha)} \delta_{j k}\right) \psi_{k}^{(\alpha)}-\frac{1}{4}\left(F_{a}^{\mu \nu} F_{\mu \nu}^{a}\right)
$$

a partir da qual são obtidas as regras de Feynman para a QCD, o que permite o cálculo de processos. A soma é feita pelo índice de sabor $\alpha=u, d, s, \ldots$, pelos índices de cor $j, k=1,2,3$ e pelo índice do campo do glúon $a=1, \ldots, 8$, sendo a derivada covariante expressa por:

$$
\mathbf{D}_{\mu} \psi=\left(\partial_{\mu}+i g A_{\mu}^{a} \frac{\lambda_{a}}{2}\right) \psi
$$

onde $\lambda_{a}$ são as matrizes de Gell-Mann, geradores de $S U(3)$ na representação fundamental. Os quarks são atribuídos à representação fundamental do grupo, enquanto o glúon é atribuído à representação adjunta. Vale também:

$$
F_{\mu \nu}^{a}=\partial_{\mu} A_{\nu}^{a}-\partial_{\nu} A_{\mu}^{a}-g f^{a b c} A_{\mu}^{b} A_{\nu}^{c},
$$

sendo $f^{a b c}$ as constantes de estrutura de $S U(3)$.

Nota-se, em particular, que o acoplamento $g$ é o mesmo para todos os sabores dos quarks. Na verdade, a expressão de sabor na QCD se dá apenas no aumento do número de quarks (na soma 2.43), o sabor dos quarks não tem influência alguma na interação da QCD. Como a soma sobre os sabores, na equação (2.43), ocorre para produtos de termos com o mesmo sabor, notando também que os geradores da QCD não misturam índices de sabor, segue que a QCD não apresenta troca de sabor (a nível árvore).

A QCD possui muitas características peculiares [2] como a liberdade assintótica, que significa que partículas que sofrem esta interação, a altas energias, comportam-se como se fossem livres; o confinamento dos quarks, que impossibilita a observação de quarks isolados, entre outras características muito importantes, que tornaram indispensável a inclusão desta teoria no Modelo Eletrofraco de Glashow-Weinberg-Salam. Nesta tese, entretanto, a atenção será voltada a aspectos da QCD que, em teorias extra-dimensionais, possam afetar a física de sabor. 


\subsection{O Bóson de Higgs}

A única partícula escalar fundamental do Modelo Padrão é o bóson de Higgs. Esta partícula é a única que ainda não foi descoberta. Observando novamente seu potencial de auto-interação, em particular o segundo termo do lado direito da equação 2.14, nota-se que, quando o campo de Higgs obtém um valor esperado de vácuo igual a $v / \sqrt{2}$, o coeficiente de acoplamento pode ser expresso, em relação à massa do bóson de Higgs, como [2]:

$$
\lambda=\frac{1}{2} \frac{M_{H}^{2}}{v^{2}} .
$$

Ou seja para $M_{H} \gg v$ o Higgs se torna fortemente acoplado.

Pode-se estimar quando a teoria se torna fortemente acoplada de duas maneiras. A primeira é observando a largura do bóson de Higgs. Quando a massa do Higgs é muito grande, os modos dominantes de seus decaimentos são:

$$
H^{0} \rightarrow W^{+} W^{-}
$$

com largura

$$
\Gamma_{H^{0} \rightarrow W^{+} W^{-}}=\frac{1}{16 \pi} \frac{M_{H}^{3}}{v^{2}} \sqrt{1-4 \frac{M_{W}}{M_{H}}}\left(1-4\left(\frac{M_{W}}{M_{H}}\right)^{2}+12\left(\frac{M_{W}}{M_{H}}\right)^{4}\right)
$$

e o modo:

$$
H^{0} \rightarrow Z^{0} Z^{0}
$$

com largura

$$
\Gamma_{H^{0} \rightarrow Z^{0} Z^{0}}=\frac{1}{32 \pi} \frac{M_{H}^{3}}{v^{2}} \sqrt{1-4 \frac{M_{Z}}{M_{H}}}\left(1-4\left(\frac{M_{Z}}{M_{H}}\right)^{2}+12\left(\frac{M_{Z}}{M_{H}}\right)^{4}\right)
$$

que se combinam para fornecer a largura do bóson de Higgs massivo:

$$
\Gamma_{H^{0} \rightarrow V V}=\frac{3}{32 \pi} \frac{M_{H}^{3}}{v^{2}},
$$

onde as contribuições dos decaimentos em férmions foram desconsideradas pois são proporcionais à massa do Higgs, enquanto os decaimentos em bósons vetoriais são proporcionais à massa ao cubo. 
Desta maneira, se $M_{H} \sim 1,4 \mathrm{TeV}$, a largura do Higgs é igual à sua massa, uma característica de interação forte e que leva ao questionamento se o Higgs é, de fato, uma ressonância. Se o Higgs está fortemente acoplado, talvez não esteja presente na teoria como uma partícula de fato, mas possivelmente como um condensado ou outro tipo de partícula que simplesmente não se pode identificar, por falhar a teoria de perturbações, principal instrumento de investigação fenomenológica. Pode ser questionado se o Higgs é, de fato, um grau de liberdade da teoria e se deveria ser incluído na sua lagrangeana inicialmente. Nota-se que $M_{H}=1,4 \mathrm{TeV}$, corresponde ao acoplamento $\lambda \sim 16$.

A segunda maneira de se estimar se a teoria é fortemente acoplada é a chamada violação de unitariedade. O vínculo de unitariedade deve ser satisfeito por qualquer elemento de matriz $\mathrm{S}$, para todos os valores de massa e constantes de acoplamento [2]. Contudo, só será satisfeito se a teoria for tratada em todas as ordens de teoria de perturbações. Uma aproximação perturbativa não necessita obedecer unitariedade numa dada ordem.

O vínculo de unitariedade para a matriz T, a nível árvore, sobre o espalhamento de onda $\mathrm{S}$ de bósons $Z^{0}$ longitudinais [2] é dado por:

$$
\left|T_{0}\right|^{2}<\frac{s}{s-4 M_{Z}^{2}}
$$

enquanto para $s \gg M_{H}^{2} \gg M_{W}^{2}$ o elemento de matriz T se torna:

$$
T_{0} \rightarrow-\frac{3}{16 \pi} \frac{M_{H}^{2}}{v^{2}}
$$

o que mostra que a unitariedade seria violada para $M_{H} \sim 1 \mathrm{TeV}$, com $s \gg M_{Z}^{2}$ a nível árvore, para a onda S. Isto, por sua vez, apesar de não significar falha da teoria, indica problemas com o cálculo de nível árvore e, portanto, sugere que a teoria de perturbações falha para tais energias. Desta maneira esperase um limite superior para a massa do bóson de Higgs de cerca de $1 \mathrm{TeV}$.

Por outro lado, o bóson de Higgs é o único escalar fundamental da teoria. As correções quânticas para a massa (ao quadrado) de um escalar, a um loop, divergem quadraticamente com o corte da teoria. Assim, as correções à massa do bóson de Higgs a um loop, uma das quais mostrada na figura 2.1, levam a [12]:

$$
\Delta M_{H}^{2} \sim \Lambda^{2} \frac{3\left(2 M_{W}^{2}+M_{Z}^{2}+M_{H}^{2}-4 m_{t}^{2}\right)}{32 \pi^{2} v^{2}}
$$


mais termos logaritmicamente dependentes do corte.

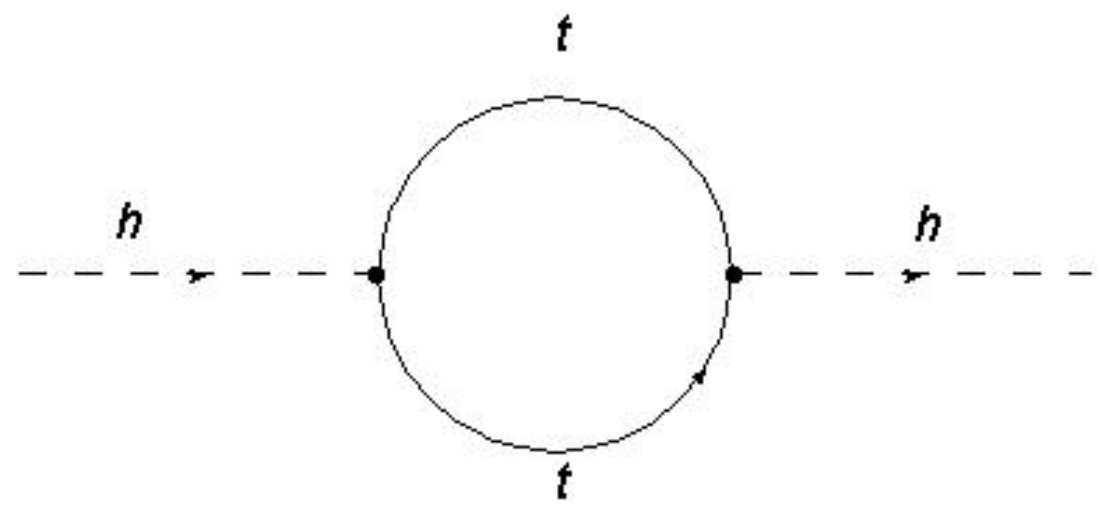

Figura 2.1: Correção quântica à massa do bóson de Higgs.

Como foi mencionado, o Modelo Padrão inclui três das quatro interações fundamentais conhecidas, faltando apenas a gravidade. Para se perceber efeitos quânticos da gravitação e, portanto, para que se deva incluí-la no Modelo Padrão, são necessárias energias da ordem da massa de Planck

$$
M_{P}=1,22089(6) \times 10^{19} \mathrm{GeV} .
$$

Assim, como apenas uma das interações conhecidas falta no modelo, esperase o corte da teoria $\Lambda \sim M_{P}$.

A massa do bóson de Higgs obedece, após as correções quânticas, a expressão:

$$
M_{H}^{2}=M_{0}^{2}+\Delta M_{H}^{2},
$$

onde $M_{0}$ é o parâmetro de massa do Higgs inserido na lagrangeana (bare mass). Nota-se que este parâmetro deve cancelar o excesso à massa do Higgs introduzido pelas correções quânticas, de forma que resulte, do lado esquerdo, a massa do Higgs ao quadrado.

Se o corte da teoria for cerca de $1 \mathrm{TeV}$, a massa obtida para o Higgs será da mesma ordem, se o parâmetro de massa também o for, o que significaria que haveria nova física não distante da escala TeV. Quanto maior for o corte da teoria, maior deve ser o cancelamento entre parâmetro de massa e correções quânticas, de modo que resulte uma massa para o Higgs não superior a 1 $\mathrm{TeV}$. Desta forma, para cortes a energias mais elevadas, deve haver ajuste 
fino entre as duas quantidades $M_{0}^{2}$ e $\Delta M_{H}^{2}$, pois ambas serão grandes e muito próximas, de tal maneira que a soma das duas dê lugar a um cancelamento muito grande e resulte a massa do bóson de Higgs inferior a $1 \mathrm{TeV}$. Este é o caso quando se coloca o corte da teoria na massa de Planck.

A razão da diferença entre os módulos do parâmetro de massa $\left|M_{0}^{2}\right|$ e das correções $\left|\Delta M_{H}^{2}\right|$ e as próprias correções é, neste caso extremo:

$$
\Delta=\left|\frac{\left|M_{0}^{2}\right|-\left|\Delta M_{H}^{2}\right|}{\Delta M_{H}^{2}}\right| \sim \frac{M_{H}^{2}}{M_{P}^{2}} \sim 10^{-32}
$$

ou seja, a diferença relativa entre parâmetro de massa e correções quânticas é, neste caso, $10^{32}$ vezes menor do que quaisquer das duas grandezas. Isto poderia não representar problema, entretanto as duas grandezas em questão não são relacionadas pela teoria, faltando uma explicação para uma similaridade tão grande entre duas grandezas de origens distintas. O problema é equivalente à falta de explicação entre a diferença entre a escala eletrofraca (massa do Higgs, cerca de $1 \mathrm{TeV}$ ) e a massa da Planck, que ficou conhecido como problema da hierarquia e é um grande motivador das pesquisas teóricas em física de partículas elementares, buscando-se através de sua solução teorias que estendam o Modelo Padrão.

\subsection{Extensões do Modelo Padrão}

Extensões do Modelo Padrão devem se reduzir a ele a baixas energias, dado seu sucesso nos experimentos como LEP e TEVATRON. Há enorme variedade de teorias e mais surgem a cada dia. As principais extensões estudadas atualmente são aquelas que resolvem o problema da hierarquia, porque predizem nova física ao alcance do LHC. Estas são, entre outras, a supersimetria, technicolor e teorias com dimensões extras.

\subsubsection{Supersimetria}

A Supersimetria [13, 14, 15, 16], [17] é a única extensão do grupo de Poincaré, introduzindo na teoria, para cada partícula existente, um parceiro supersimétrico correspondente que possui diferença de spin para a partícula original em meia unidade. Assim, aos férmions correspondem os escalares 
chamados sférmions, aos bósons de gauge, férmions chamados gauginos e, considerando apenas a extensão do Modelo Padrão à supersimetria, ao bóson de Higgs, o férmion denominado higgsino.

Como nenhum parceiro supersimétrico foi observado até o presente, a supersimetria deve estar quebrada. Se a supersimetria é quebrada em energias não muito altas comparadas com a escala eletrofraca (e.g. $<1 \mathrm{TeV}$ ), os estados supersimétricos cancelam as divergências quadráticas na massa do bóson de Higgs, estabilizando deste jeito a escala eletrofraca e resolvendo o problema da hierarquia.

Algumas características da supersimetria são: melhor unificação das constantes de acoplamento na escala de grande unificação e candidatos naturais a matéria escura, por exemplo. Entretanto a teoria se torna menos promissora devido à ausência de resultados experimentais que mostrem estados supersimétricos. Há também a enorme quantidade de parâmetros de um possível Modelo Padrão Supersimétrico Mínimo (MSSM), parâmetros não explicados pela teoria. A supersimetria continua sendo objeto de intensa pesquisa e, no contexto desta tese, pode ser uma ferramenta interessante para extensão dos modelos que serão contruídos.

\subsubsection{Technicolor}

As teorias de Technicolor (TC) [18, 19, 20], [21] são baseadas na existência de novas interações de gauge, responsáveis pela condensação de novos férmions (techniquarks) e resultando na quebra dinâmica da simetria eletrofraca, pois essas interações se tornam fortes para energias $\mathcal{O}(1 \mathrm{TeV})$. Esse fato tem a vantagem de eliminar a necessidade de um bóson de Higgs fundamental, que até hoje não foi observado, pois o condensado de férmions faz seu papel. Isto resolve também o problema da hierarquia, pois sem escalares elementares não há as divergências do MP e, portanto, não há ajuste fino.

Entretanto, para gerar as massas dos férmions, o grupo de gauge do Technicolor deve ser estendido, gerando o chamado Technicolor estendido (ETC) $[22,23]$. Mas, devido aos bósons de gauge do technicolor estendido, aparecem interações que violam vínculos experimentais, em particular surgem correntes neutras que trocam sabor (FCNC). Como no caso da supersimetria, pode ser 
interessante utilizar aspectos de technicolor na construção de modelos, bem como, modificar a teoria para aplicações futuras.

No capítulo 4 é introduzido um mecanismo de condensação de quarks [8] onde, devido a interações não universais, é obtida a condensação de quarks, com troca de sabor a nível árvore mas sem a violação de vínculos experimentais da física de sabor.

\subsubsection{Dimensões Extras}

A extensão do Modelo Padrão pode ser construída com o uso de dimensões extras [24, 25]. Estas podem ser planas ou curvas e ser em número arbitrário, desde que não sejam observáveis seus efeitos nas escalas de energia testadas até o presente. Dimensões extras resolvem o problema da hierarquia porque o corte do Modelo Padrão fica na escala eletrofraca (UED - Universal Extra Dimensions) ou é trazido a esta escala pela estrutura do espaço (LED Large Extra Dimensions e WED - Warped Extra Dimensions), eliminando naturalmente a necessidade de ajuste fino [26].

O fato de não haverem sido observados efeitos de dimensões adicionais até o momento sugere que estas são compactas, o que significa que qualquer dimensão extra não pode ser infinita e, parametrizando qualquer de suas coordenadas por $y$, deve valer $y+L=y$ para algum comprimento $L$ não observável nas escalas de energias já exploradas. Isto significa que a dimensão extra deve ser pequena.

A métrica para uma teoria com dimensões planas é dada por:

$$
d s^{2}=g_{\mu \nu}(x) d x^{\mu} d x^{\nu}-\delta_{a b} d y^{a} d y^{b}
$$

sendo $g_{\mu \nu}$ o tensor métrico 4-dimensional e $y^{a}$ denota uma das coordenadas das dimensões adicionais.

Como as dimensões extras não são percebidas na escala macroscópica, devem ser compactas, requisito que leva a condições de contorno para campos: $\phi(y)=\phi(y+2 \pi R)$, para algum $\mathrm{R}$ pequeno $(L=2 \pi R)$.

Para ilustrar técnicas envolvidas e propriedades de teorias extra-dimensionais, seja um espaço plano com uma dimensão extra e um campo escalar que, de- 
vido às condições de contorno, pode ser expandido em série de Fourier:

$$
\begin{array}{r}
\phi(x, y)=\frac{1}{\sqrt{2 \pi R}} \phi_{0}(x)+\sum_{n=1}^{\infty} \frac{1}{\sqrt{\pi R}}\left[\phi_{n}(x) \cos \left(\frac{n y}{R}\right)+\right. \\
\left.+\hat{\phi}_{n}(x) \sin \left(\frac{n y}{R}\right)\right] .
\end{array}
$$

O termo $\phi_{0}$, que não depende da quinta dimensão, é chamado de modo zero. Os outros modos de Fourier $\phi_{n}$ e $\hat{\phi}_{n}$ são chamados de modos de Kaluza-Klein (KK). A ação é:

$$
S[\phi]=\frac{1}{2} \int d^{4} x d y\left(\partial^{A} \phi \partial_{A} \phi-m^{2} \phi^{2}\right),
$$

onde $A=0,1,2,3,5$.

Substituindo a expansão (2.55) na expressão (2.56) e integrando pela dimensão extra resulta:

$$
\begin{aligned}
S[\phi]= & \frac{1}{2} \sum_{n=0}^{\infty} \int d^{4} x\left(\partial^{\mu} \phi_{n} \partial_{\mu} \phi_{n}-m_{n}^{2} \phi_{n}^{2}\right)+ \\
& +\frac{1}{2} \sum_{n=1}^{\infty} \int d^{4} x\left(\partial^{\mu} \hat{\phi}_{n} \partial_{\mu} \hat{\phi}_{n}-m_{n}^{2} \hat{\phi}_{n}^{2}\right) .
\end{aligned}
$$

Isso mostra que a quinta dimensão é percebida, na teoria efetiva 4-dimensional, como um espectro de partículas, onde a massa dos modos KK é dada por $m_{n}^{2}=m^{2}+n^{2} / R^{2}$. Desta maneira, para que se detecte um modo excitado de um campo, é necessário utilizar energias maiores do que $m_{n}^{2}$, neste caso tornam-se disponíveis para observação as partículas correspondentes aos modos $0,1,2, \ldots, n$. Todos os modos de Kaluza-Klein possuem os mesmos números quânticos de $\phi_{0}$.

Uma outra abordagem utilizando dimensões extras é o uso de espaço curvo, o que é feito a seguir, em maior detalhe, onde são analisados campos em espaço com uma dimensão extra curva. 


\section{Capítulo 3}

\section{Espaço Curvo com Cinco Dimensões}

Uma das possibilidades de extensão do Modelo Padrão (MP) é a adição de dimensões extras às quatro usuais. Desconsiderando-se efeitos gravitacionais, o espaço de Minkowski se torna um sub-espaço deste novo espaço estendido.

Sendo o Modelo Padrão válido dentro dos limites até o presente explorados, deve ser obtido como limite de qualquer teoria que lhe sirva de extensão. Desta forma, devem ser buscadas representações de bósons e férmions, em teorias com dimensões extras compactas, que possam acomodar as partículas do MP. Uma das possibilidades, a que será detalhada no presente trabalho, é um espaço curvo com dimensões extras, teoria do tipo WED (Warped Extra Dimensions) [4].

Em particular, o espaço a ser estudado tem cinco dimensões (uma adicional), a métrica é do tipo anti-de Sitter (AdS), o que permite gerar a escala eletrofraca a partir da escala de Planck, caso o bóson de Higgs esteja localizado próximo a um dos pontos fixos do espaço compactificado. Todos os campos podem se propagar pela dimensão extra. O fato de se obter a escala eletrofraca a partir da escala de Planck implica que correções quânticas à massa do bóson de Higgs tem corte na escala eletrofraca, naturalmente resolvendo o problema da hierarquia. 


\subsection{O Espaço}

O espaço é composto pelas quatro dimensões do espaço de Minkowski (uma temporal e três espaciais), além de uma dimensão espacial adicional. Esta dimensão não pode ser infinita como as outras, deve estar compactificada, uma vez que sua presença não foi detectada em nenhum dos experimentos realizados até hoje. Por esse mesmo motivo deve ser muito pequena, tal que seus efeitos somente sejam observáveis para energias superiores às já sondadas.

Seja y a coordenada correspondente à quinta dimensão. Vale, para algum $R$ (denominado raio de curvatura):

$$
y+2 \pi R=y .
$$

Ou seja, para especificar a coordenada periódica y basta limitar o intervalo (com condições de contorno periódicas) a:

$$
-\pi R \leq y \leq \pi R
$$

Além disso, para que haja férmions quirais no Modelo Padrão, deve haver a identificação $\left(x^{\mu}, y\right)$ com $\left(x^{\mu},-y\right)$, isto é, os termos da lagrangeana de qualquer teoria devem respeitar a paridade $y \rightarrow-y$.

A dimensão extra é compactificada em orbifold, como ilustrado na figura 3.1. Nota-se que esta compactificação significa que a dimensão extra é um espaço $S^{1} / Z_{2}$, o que por sua vez implica que a ação fica completamente especificada para $0 \leq y \leq \pi R$. Outra consequência direta é a presença de dois pontos fixos do orbifold: $y^{*}=0$ e $y^{*}=\pi R$, que são a localização das duas 3-branas (sub-espaços quadridimensionais). Serão denominadas brana UV (Planck) e brana IR (TeV), respectivamente.

Seguindo o modelo proposto na referência [4], uma solução para as equações de Einstein em cinco dimensões, que respeita a invariância de Poincaré nas direções $x^{\mu}$, é dada por:

$$
d s^{2}=e^{-2 \sigma} \eta_{\mu \nu} d x^{\mu} d x^{\nu}-d y^{2}
$$

onde:

$$
\sigma=k|y|
$$




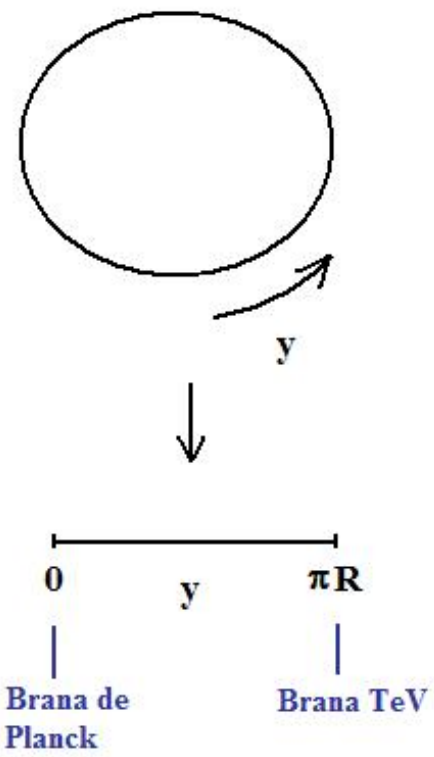

Figura 3.1: Compactificação em orbifold.

$k$ é a curvatura do espaço e:

$$
\eta_{\mu \nu}=\eta^{\mu \nu}=\left(\begin{array}{cccc}
1 & 0 & 0 & 0 \\
0 & -1 & 0 & 0 \\
0 & 0 & -1 & 0 \\
0 & 0 & 0 & -1
\end{array}\right)
$$

Os índices $\mu, \nu$ variam de 0 a 3 , a coordenada temporal corresponde ao índice 0 .

A métrica pode ser reescrita como:

$$
d s^{2}=d x^{M} d x^{N} g_{M N}=d x^{\mu} d x^{\nu} g_{\mu \nu}-d y^{2},
$$

onde os índices $M, N$ percorrem os valores 0 a 3 e 5 , sendo 5 o índice da coordenada $y$. Desta forma, o produto escalar $A_{M} B^{M}=A_{0} B^{0}+A_{1} B^{1}+$ $A_{2} B^{2}+A_{3} B^{3}+A_{5} B^{5}$. Também fica definido o tensor métrico:

$$
g_{\mu \nu}=e^{-2 \sigma} \eta_{\mu \nu}
$$

O fator exponencial é o responsável pela mudança nas escalas de energias entre os dois pontos fixos e esta é a razão de sua introdução na métrica. A 
escala de massa na brana UV é a massa de Planck:

$$
M_{P}=G_{N}^{-1 / 2} \approx 1,2 \times 10^{19} \mathrm{GeV}
$$

e, para que a escala de massa efetiva $M_{P} e^{-\pi k R}$ esteja na escala $\mathrm{TeV}$, na brana IR, deve valer $k R \approx 12$, pois para $k R=12, M_{P} e^{-\pi k R} \approx 0,5 \mathrm{TeV}$, o que claramente fica na escala TeV. Por esta razão, as branas UV e IR também são chamadas de brana de Planck e brana $\mathrm{TeV}$, respectivamente.

Assim sendo, fixando a escala de energia da teoria próxima a escala da massa da Planck $k \lesssim M_{P}$ e notando que $k \sim 10 / R$, resulta que $R^{-1}$ não difere muito de $M_{P}$, ou seja, a dimensão extra é extremamente pequena.

\subsection{Os Campos}

Uma vez caracterizado o espaço, é necessário incluir os campos da teoria. Serão tratados campos escalares, espinoriais e vetoriais, elementos necessários para a obtenção do Modelo Padrão a baixas energias a partir desta teoria.

\subsubsection{Campo Escalar}

O caso mais simples é o do campo escalar [7, 5], cuja ação é dada por:

$$
S_{e}=\int d^{4} x \int d y \sqrt{g}\left(\left|D_{M} \Phi\right|^{2}-m_{\Phi}^{2}|\Phi|^{2}\right)
$$

onde

$$
g=\operatorname{det}\left(\mathbf{g}_{M N}\right)
$$

$\mathbf{g}_{M N}$ é a representação matricial do tensor métrico covariante e $D_{M}$ é a derivada covariante. A ação pode ser reescrita como:

$$
S_{e}=\int d^{4} x \int d y \sqrt{g}\left[\left(D_{\mu} \Phi\right)^{*} D_{\nu} \Phi g^{\mu \nu}-\left(D_{5} \Phi\right)^{*} D_{5} \Phi-m_{\Phi}^{2}|\Phi|^{2}\right] .
$$

Por simplicidade notacional será considerado o campo escalar livre (posteriormente interações serão incluídas na teoria), o que resulta em $D_{\mu}=\partial_{\mu}$ e $D_{5}=\partial_{5} \equiv \partial_{y}$. 
A variação da ação é:

$$
\begin{array}{r}
\delta S_{e}=\int d^{4} x \int d y \sqrt{g}\left\{\frac{\delta}{\delta \partial_{\rho} \Phi^{*}}\left(\partial_{\mu} \Phi^{*} \partial_{\nu} \Phi g^{\mu \nu}\right) \delta\left(\partial_{\rho} \Phi^{*}\right)+\right. \\
+\frac{\delta}{\delta \partial_{\rho} \Phi}\left(\partial_{\mu} \Phi^{*} \partial_{\nu} \Phi g^{\mu \nu}\right) \delta\left(\partial_{\rho} \Phi\right)-\frac{\delta}{\delta \partial_{y} \Phi^{*}}\left[\partial_{y} \Phi^{*} \partial_{y} \Phi\right] \delta\left(\partial_{y} \Phi^{*}\right)- \\
\left.-\frac{\delta}{\delta \partial_{y} \Phi}\left(\partial_{y} \Phi^{*} \partial_{y} \Phi\right) \delta\left(\partial_{y} \Phi\right)-m_{\Phi}^{2}\left[\frac{\delta}{\delta \Phi^{*}}\left(\Phi^{*} \Phi\right) \delta \Phi^{*}+\frac{\delta}{\delta \Phi}\left(\Phi^{*} \Phi\right) \delta \Phi\right]\right\} .
\end{array}
$$

Como é usual, cada campo $\Phi$ e $\Phi^{*}$ é independente (pois podem ser decompostos em dois campos reais independentes). Usando:

$$
\frac{\delta}{\delta \partial_{\nu} \Phi} \partial_{\mu} \Phi=\delta_{\mu}^{\nu}
$$

resulta:

$$
\begin{array}{r}
\delta S_{e}=\int d^{4} x \int d y \sqrt{g}\left\{\partial_{\nu} \Phi g^{\mu \nu} \partial_{\mu} \delta \Phi^{*}+\partial_{\mu} \Phi^{*} g^{\mu \nu} \partial_{\nu} \delta \Phi-\right. \\
\left.-m_{\Phi}^{2}\left(\Phi \delta \Phi^{*}+\Phi^{*} \delta \Phi\right)-\partial_{y} \Phi \partial_{y}\left(\delta \Phi^{*}\right)-\partial_{y} \Phi^{*} \partial_{y}(\delta \Phi)\right\}
\end{array}
$$

A regra do produto para derivadas leva a:

$$
\begin{aligned}
& \int d^{4} x \int d y \sqrt{g} \partial_{\nu} \Phi g^{\mu \nu} \partial_{\mu} \delta \Phi^{*}=\int d^{4} x \int d y\left\{\partial_{\mu}\left(\sqrt{g} \partial_{\nu} \Phi g^{\mu \nu} \delta \Phi^{*}\right)-\right. \\
& \left.-\partial_{\mu}\left(\sqrt{g} \partial_{\nu} \Phi g^{\mu \nu}\right) \delta \Phi^{*}\right\}
\end{aligned}
$$

e

$$
\int d^{4} x \int d y \sqrt{g} \partial_{y} \Phi \partial_{y}\left(\delta \Phi^{*}\right)=\int d^{4} x \int d y\left\{\begin{array}{r}
\partial_{y}\left(\sqrt{g} \partial_{y} \Phi \delta \Phi^{*}\right)- \\
\left.-\partial_{y}\left(\sqrt{g} \partial_{y} \Phi\right) \delta \Phi^{*}\right\}
\end{array}\right.
$$

Desta última equação, integrando-se o primeiro termo do lado direito da equação em $y$, entre $a$ e $b$ (os limites de integração da variável), resulta:

$$
\begin{array}{r}
\int d^{4} x \int d y \sqrt{g} \partial_{y} \Phi \partial_{y}\left(\delta \Phi^{*}\right)=-\int d^{4} x \int d y \partial_{y}\left(\sqrt{g} \partial_{y} \Phi\right) \delta \Phi^{*}+ \\
+\left.\int d^{4} x\left[\sqrt{g} \partial_{y} \Phi \delta \Phi^{*}\right]\right|_{y=a} ^{y=b},
\end{array}
$$

enquanto aplicando-se o teorema de Gauss em quatro dimensões ao primeiro termo do lado direito da equação (3.14), para a integral nas quatro dimensões 
$x^{\mu}$, nota-se que o termo é nulo. Isto ocorre porque cada dimensão do espaço de Minkowski quadridimensional é infinita e, portanto, a integral de superfície resultante deve se anular para campos que se anulam suficientemente rápido no infinito, como está sendo assumido que seja o caso.

Assim, para a variação da ação se obtém:

$$
\begin{array}{r}
\delta S_{e}=\int d^{4} x \int d y\left\{\delta \Phi\left[\partial_{y}\left(\sqrt{g} \partial_{y} \Phi^{*}\right)-\sqrt{g}\left(m_{\Phi}^{2}+\partial_{\mu} \partial_{\nu} g^{\mu \nu}\right) \Phi^{*}\right]+\right. \\
\left.+\delta \Phi^{*}\left[\partial_{y}\left(\sqrt{g} \partial_{y} \Phi\right)-\sqrt{g}\left(m_{\Phi}^{2}+\partial_{\mu} \partial_{\nu} g^{\mu \nu}\right) \Phi\right]\right\}- \\
-\left.\int d^{4} x\left\{\sqrt{g}\left[\partial_{y} \Phi \delta \Phi^{*}+\partial_{y} \Phi^{*} \delta \Phi\right]\right\}\right|_{y=a} ^{y=b}
\end{array}
$$

Para valores arbitrários de $\delta \Phi^{*}$ :

$$
\sqrt{g}\left(m_{\Phi}^{2}+g^{\mu \nu} \partial_{\mu} \partial_{\nu}\right) \Phi-\partial_{y}\left(\sqrt{g} \partial_{y} \Phi\right)=0
$$

Das equações (3.7) e (3.10) resulta:

$$
g=\operatorname{det}\left(\mathbf{g}_{M N}\right)=e^{-8 \sigma}
$$

que implica que:

$$
\sqrt{g}=e^{-4 \sigma}
$$

Levando em consideração a compactificação em orbifold, todos os termos da densidade de lagrangeana devem ser pares, sob a transformação $y \rightarrow-y$, o que equivale a integrar em $y$ entre $a=0$ e $b=\pi R$ em lugar de $a=-\pi R$, mediante a multiplicação por dois da integral. Tomando esses limites, os valores da equação (3.17) ficam dobrados, o que não interfere com a equação de movimento (3.18) ou com as condições de contorno do último termo de (3.17).

Como o intervalo foi restrito a $0 \leq y \leq \pi R$, o último termo da equação (3.18) se torna:

$$
\partial_{y}\left(\sqrt{g} \partial_{y} \Phi\right)=-4 k e^{-4 k y} \partial_{y} \Phi+e^{-4 k y} \partial_{y}^{2} \Phi
$$

Usando a igualdade $g^{M N} g_{N P}=\delta_{P}^{M}$, a equação de movimento (3.18) pode ser ainda simplificada. Da equação (3.7) se obtém:

$$
\left(\partial_{\mu} \partial^{\mu}+e^{-2 k y} m_{\Phi}^{2}\right) \Phi-e^{2 k y} \partial_{y}\left(e^{-4 k y} \partial_{y} \Phi\right)=0
$$


e para as condições de contorno, do termo de superfície da equação (3.17) resulta:

$$
\left.\int d^{4} x\left(\sqrt{g} \partial_{y} \Phi \delta \Phi^{*}\right)\right|_{y=0} ^{y=\pi R}=0
$$

Para a solução da equação de movimento, é adotado o Ansatz:

$$
\Phi\left(x^{\mu}, y\right)=\frac{1}{\sqrt{\pi R}} \sum_{n} \phi^{(n)}(x) \psi^{(n)}(y)
$$

sobre a separação de variáveis, com:

$$
\left(\partial_{\mu} \partial^{\mu}+m_{n}^{2}\right) \phi^{(n)}(x)=0
$$

e a condição de normalização:

$$
\frac{1}{\pi R} \int_{0}^{\pi R} d y e^{-2 k y} \psi^{(n)}(y) \psi^{(m)}(y)=\delta_{m n},
$$

para que a ação, após a integral em y, seja a ação para campos de KleinGordon.

A substituição do Ansatz na equação de movimento (3.20) leva a:

$$
\sum_{n}\left[\left(\partial_{\mu} \partial^{\mu}+m_{\Phi}^{2} e^{-2 k y}\right) \phi^{(n)} \psi^{(n)}-e^{-2 k y} \phi^{(n)}\left(\partial_{y}-4 k\right) \partial_{y} \psi^{(n)}\right]=0 .
$$

Cada termo $\phi^{(n)}(x)$ na expansão (3.22) é um campo 4-dimensional denominado modo $\mathrm{n}$ de Kaluza-Klein (modo KK) do campo $\Phi$ e da partícula correspondente. A função $\psi^{(n)}(y)$, dependente de $y$, é denominada função de onda do modo $n$ na dimensão extra. Após a integração da dimensão adicional, restarão, como sinal de dimensão extra, modos KK de um mesmo campo, relacionados pelas equações de movimento e condições de contorno, representando partículas com números quânticos idênticos.

À medida que aumenta o valor de $n$, aumenta também o de $m_{n}$, como será observado adiante, o que significa que maiores energias devem ser fornecidas ao sistema para que os modos KK superiores possam ser detectados. A cada modo KK corresponde uma partícula com números quânticos idênticos aos do campo $\Phi$, mas com massa distinta.

Assim, como se busca a obtenção do Modelo Padrão como limite de baixas energias da teoria, é necessário estudar as propriedades das partículas sem 
massa, os chamados modos zero. A interpretação que se faz é que as partículas observadas do Modelo Padrão correspondem aos modos zero dos campos em cinco dimensões. Portanto os modos zero devem ser estudados com mais cuidado.

Para se encontrar o modo zero é utilizada a definição $m_{n}=0$ e $n=0$ pois o parâmetro $m_{n}$ representa uma contribuição à massa da partícula quando a dimensão adicional é eliminada, por integração. Como o bóson de Higgs é o responsável pela geração de massas para as partículas do MP, para estas, não deve haver massa inicialmente na lagrangeana, antes de se inserir o bóson de Higgs na teoria e, portanto, $m_{n}$ deve se anular, se o modo zero representa campo do MP.

O modo zero corresponde a $n=0 \mathrm{e}$, apesar de haver a interpretação acima descrita, não são todos os campos em cinco dimensões que possuem modo zero, mas aqueles que correspondem a campos do MP certamente devem possuir.

Para o modo zero, obtém-se, a partir da equação (3.25) e da (3.23):

$$
\frac{d^{2}}{d y^{2}} \psi^{(0)}-4 k \frac{d}{d y} \psi^{(0)}-m_{\Phi}^{2} \psi^{(0)}=0,
$$

que tem solução:

$$
\psi^{(0)}(y)=c_{1} e^{(2-\alpha) k y}+c_{2} e^{(2+\alpha) k y}
$$

onde:

$$
\alpha=\sqrt{4+\frac{m_{\Phi}^{2}}{k^{2}}} .
$$

e com o cuidado de usar a solução (3.27) quando $\alpha \neq 0$, caso contrário deve-se solucionar novamente a equação (3.26). Este caso não será trabalhado aqui pois requer valores negativos de $m_{\Phi}^{2}$.

Caso valha $m_{\Phi}=0$ nota-se, após a aplicação das condições de contorno, que a função de onda $\psi(y)$ é apenas uma constante. Este caso também não será abordado para escalares, pois, como será visto, implica em acoplamentos $5 \mathrm{D}$ universais com os férmions o que por sua vez resultaria na manutenção do problema da hierarquia das massas (dos férmions). O único escalar elementar do MP é o bóson de Higgs, que tem acoplamentos não universais, isto é $m_{\Phi} \neq 0$. 
Assim, para $\alpha>2$, as variações arbitrárias de $\Phi$ nas branas, aplicadas à equação (3.21), resultam em condições de contorno de Neumann:

$$
\left.\frac{d}{d y} \psi^{(0)}\right|_{y=0}=\left.\frac{d}{d y} \psi^{(0)}\right|_{y=\pi R}=0
$$

Pode-se observar, aplicando à equação (3.27) as condições de contorno, que estas implicam que $c_{1}=c_{2}=0$. Ou seja, o campo não possui modo zero. Caso fossem usadas condições de Dirichlet, a conclusão seria a mesma.

Para se obter campo com modo zero, a ação, equação (3.9), deve ser modificada pela inclusão de um termo de superfície nas branas, por exemplo:

$$
S_{\text {brana }}=\int d^{4} x \int d y \sqrt{g} b k[\delta(y)-\delta(y-\pi R)]|\Phi|^{2},
$$

que, com a variação da ação modificada, leva às novas condições de contorno para o modo zero:

$$
\left.\left(\frac{d}{d y} \psi^{(0)}-b k \psi^{(0)}\right)\right|_{y=0} ^{y=\pi R}=0
$$

Por fim, aplicando essas condições de contorno modificadas à solução (3.27) resultam:

$$
(2-\alpha-b) c_{1}+(2+\alpha-b) c_{2}=0
$$

e

$$
(2-\alpha-b) c_{1} e^{(2-\alpha) \pi k R}+(2+\alpha-b) c_{2} e^{(2+\alpha) \pi k R}=0 .
$$

Para valores genéricos de $\alpha$ e $b$ resulta novamente que $c_{1}=c_{2}=0$. Entretanto, se $b=2-\alpha \rightarrow c_{2}=0$ e se $b=2+\alpha \rightarrow c_{1}=0$. De qualquer forma, vale:

$$
\psi^{(0)}(y) \propto e^{b k y}
$$

$\operatorname{com} b=2 \pm \alpha$.

Para os modos KK $(n>0), m_{n} \neq 0$, o que resulta, para o modo $n$, em:

$$
e^{-2 k y} \frac{d^{2}}{d y^{2}} \psi^{(n)}-4 k e^{-2 k y} \frac{d}{d y} \psi^{(n)}+\left(m_{n}^{2}-m_{\Phi}^{2} e^{-2 k y}\right) \psi^{(n)}=0,
$$

com condições de contorno:

$$
\left.\left(\frac{d}{d y} \psi^{(n)}-b k \psi^{(n)}\right)\right|_{y=0} ^{y=\pi R} .
$$


Com as transformações:

$$
z=\frac{m_{n}}{k} e^{k y}
$$

e

$$
\psi^{(n)}(y)=\frac{z^{2} k^{2}}{m_{n}^{2}} \varphi^{(n)}(z),
$$

substituídas na equação (3.35), resulta:

$$
z^{2} \frac{d^{2}}{d z^{2}} \varphi^{(n)}+z \frac{d}{d z} \varphi^{(n)}+\left(z^{2}-\alpha^{2}\right) \varphi^{(n)}=0 .
$$

Esta equação pode ser reconhecida como a equação de Bessel [27]. Sua solução leva a:

$$
\varphi^{(n)}(z)=c_{1}^{\prime} \mathrm{J}_{\alpha}(z)+c_{2}^{\prime} \mathrm{Y}_{\alpha}(z)
$$

que, com:

$$
c_{i}^{\prime}\left(\frac{m_{n}}{k}\right)^{2}=c_{i}
$$

leva a:

$$
\psi^{(n)}(y)=e^{2 k y}\left[c_{1} \mathrm{~J}_{\alpha}\left(\frac{m_{n}}{k} e^{k y}\right)+c_{2} \mathrm{Y}_{\alpha}\left(\frac{m_{n}}{k} e^{k y}\right)\right] .
$$

As condições de contorno se tornam:

$$
\begin{array}{r}
m_{n}\left[c_{1} \mathrm{~J}_{\alpha}^{\prime}\left(\frac{m_{n}}{k}\right)+c_{2} \mathrm{Y}^{\prime}{ }_{\alpha}\left(\frac{m_{n}}{k}\right)\right]+ \\
+k(2-b)\left[c_{1} \mathrm{~J}_{\alpha}\left(\frac{m_{n}}{k}\right)+c_{2} \mathrm{Y}_{\alpha}\left(\frac{m_{n}}{k}\right)\right]=0
\end{array}
$$

e

$$
\begin{array}{r}
m_{n} e^{\pi k R}\left[c_{1} \mathrm{~J}^{\prime}{ }_{\alpha}\left(\frac{m_{n}}{k} e^{\pi k R}\right)+c_{2} \mathrm{Y}^{\prime}{ }_{\alpha}\left(\frac{m_{n}}{k} e^{\pi k R}\right)\right]+ \\
+k(2-b)\left[c_{1} \mathrm{~J}_{\alpha}\left(\frac{m_{n}}{k} e^{\pi k R}\right)+c_{2} \mathrm{Y}_{\alpha}\left(\frac{m_{n}}{k} e^{\pi k R}\right)\right]=0,
\end{array}
$$

$\operatorname{com} J^{\prime}(a) \equiv d J /\left.d y\right|_{y=a}$. Estas condições determinam $m_{n}$ e $c_{1}$, por exemplo, neste caso $c_{2}$ fica determinado pela normalização.

Destas últimas, para $m_{n}>>1$, vale a aproximação, baseada nas formas assintóticas das funções de Bessel:

$$
m_{n} \approx \pi k e^{-\pi k R}\left(n+\frac{\alpha}{2}-\frac{3}{4}\right) .
$$

Da aproximação para a massa, fica claro que ela aumenta conforme aumenta $n$.

Uma vez caracterizado o campo escalar, segue a caracterização do campo espinorial, para a construção e extensão do Modelo Padrão. 


\subsubsection{Campo Espinorial}

O campo espinorial [6,5], além de mais complexo algebricamente do que o campo escalar, introduz o problema de se reproduzir a quiralidade necessária às interações fracas.

A ação do campo espinorial é dada por:

$$
S_{s}=\int d^{4} x \int d y \sqrt{g}\left(\frac{i}{2} \bar{\Psi} \gamma^{M}\left(\partial_{M}-\overleftarrow{\partial}_{M}\right) \Psi-m_{\Psi} \bar{\Psi} \Psi\right)
$$

O termo de conexão de spin não foi incluído, pois não contribui para a ação, uma vez que a métrica é diagonal.

As matrizes gama são definidas por:

$$
\gamma_{M}=E_{M}^{\alpha} \gamma_{\alpha}
$$

onde $\gamma_{\alpha}=\left(\gamma_{\mu}, i \gamma_{5}\right), \gamma_{\mu}$ são as matrizes de Dirac no espaço plano e $E_{M}^{\alpha}$ é o vierbein (ou funfbein, por ser em cinco dimensões), definido por [28]:

$$
E_{M}^{\alpha}(X)=\left.\left(\frac{\partial}{\partial x^{M}} \xi_{X}^{\alpha}(x)\right)\right|_{x=X}
$$

onde $\xi_{X}^{\alpha}$ é um sistema de coordenadas localmente inercial em $X$. A métrica em sistema não-inercial geral é:

$$
g_{M N}(x)=E_{M}^{\alpha}(x) E_{N}^{\beta}(x) \eta_{\alpha \beta},
$$

sendo $\eta_{M N}$ a métrica plana em cinco dimensões.

Desta forma, segue que

$$
E_{M}^{\alpha}=\delta_{M}^{\alpha} \cdot\left\{\begin{array}{rr}
e^{-\sigma} & M \neq 5 \\
1 & M=5
\end{array}\right.
$$

A ação, equação (3.45), torna-se:

$$
\begin{array}{r}
S_{s}=\int d^{4} x \int d y e^{-4 \sigma}\left(\frac{i}{2} \bar{\Psi} \gamma^{\mu}\left(\partial_{\mu}-\overleftarrow{\partial}_{\mu}\right) \Psi e^{\sigma}+\frac{i}{2} \bar{\Psi} i \gamma^{5}\left(\partial_{y}-\overleftarrow{\partial}_{y}\right) \Psi-\right. \\
\left.-m_{\Psi} \bar{\Psi} \Psi\right)
\end{array}
$$

Vale também:

$$
\frac{-i}{2} \bar{\Psi} \gamma^{\mu} \overleftarrow{\partial}_{\mu} \Psi e^{\sigma}=\frac{-i}{2} \partial_{\mu}\left(\bar{\Psi} \gamma^{\mu} \Psi e^{\sigma}\right)+\frac{i}{2} \bar{\Psi} \gamma^{\mu} \partial_{\mu} \Psi e^{\sigma}
$$


onde o primeiro termo do lado direito se anula, quando integrado em $d^{4} x$, com a aplicação do teorema de Gauss e a imposição de que os campos se anulem suficientemente rápido no infinito. Alem disso, vale também:

$$
\frac{-i}{2} \bar{\Psi} i \gamma^{5} \overleftarrow{\partial}_{y} \Psi=\frac{-i}{2} \partial_{y}\left(\bar{\Psi} i \gamma^{5} \Psi\right)+\frac{i}{2} \bar{\Psi} i \gamma^{5} \partial_{y} \Psi
$$

Por fim, o parâmetro de massa é escrito como:

$$
m_{\Psi}=m \operatorname{sgn}(y)
$$

A aplicação dos últimos resultados à ação resulta em:

$$
\begin{aligned}
S_{s}=\int d^{4} x \int d y e^{-4 \sigma}\left(i \bar{\Psi} \gamma^{\mu} \partial_{\mu} \Psi e^{\sigma}-\bar{\Psi} \gamma^{5} \partial_{y} \Psi\right. & +\frac{1}{2} \partial_{y}\left(\bar{\Psi} \gamma^{5} \Psi\right)- \\
& -m \operatorname{sgn}(y) \bar{\Psi} \Psi) .
\end{aligned}
$$

As transformações dos campos, sob a paridade $Z_{2}$, são dadas por:

$$
\Psi\left(x^{\mu},-y\right)= \pm \gamma^{5} \Psi\left(x^{\mu}, y\right)
$$

com o sinal determinado pelas interações dos férmions. Com esta regra de transformações, resulta que:

$$
\bar{\Psi}(-y) \Psi(-y)=\Psi^{\dagger}(y) \gamma^{5} \gamma^{0} \gamma^{5} \Psi(y)=-\bar{\Psi}(y) \Psi(y)
$$

mostrando que $\bar{\Psi} \Psi$ é ímpar e que, portanto, o parâmetro de massa $m_{\Psi}$ deve ser ímpar: $m_{\Psi}(-y)=-m_{\Psi}(y)$, para que a lagrangeana seja par, justificando a equação (3.53).

Sejam definidos os campos:

$$
\Psi_{L, R}=\frac{1}{2}\left(1 \mp \gamma^{5}\right) \Psi
$$

que ainda não são os campos quirais do Modelo Padrão, mas sim os modos zero correspondentes, como será observado. Para inserir os campos quirais na ação, é mais útil valer-se das igualdades:

$$
\bar{\Psi}_{L, R} \Psi_{L, R}=\frac{1}{4} \Psi^{\dagger}\left(1 \mp \gamma^{5}\right) \gamma^{0}\left(1 \mp \gamma^{5}\right) \Psi=\frac{1}{4} \Psi^{\dagger} \gamma^{0}\left(1 \mp \gamma^{5} \pm \gamma^{5}-\left(\gamma^{5}\right)^{2}\right) \Psi=0
$$




$$
\begin{aligned}
\bar{\Psi}_{L, R} \gamma^{\mu} \partial_{\mu} \Psi_{R, L} & =\frac{1}{4} \Psi^{\dagger}\left(1 \mp \gamma^{5}\right) \gamma^{0} \gamma^{\mu}\left(1 \pm \gamma^{5}\right) \partial_{\mu} \Psi= \\
= & \frac{1}{4} \Psi^{\dagger}\left(1 \mp \gamma^{5}\right) \gamma^{0}\left(1 \mp \gamma^{5}\right) \gamma^{\mu} \partial_{\mu} \Psi=0 \\
\bar{\Psi}_{L, R} \gamma^{5} \partial_{y} \Psi_{L, R} & =\frac{1}{4} \Psi^{\dagger}\left(1 \mp \gamma^{5}\right) \gamma^{0} \gamma^{5}\left(1 \mp \gamma^{5}\right) \Psi= \\
& =\mp \frac{1}{4} \Psi^{\dagger}\left(1 \mp \gamma^{5}\right) \gamma^{0}\left(1 \mp \gamma^{5}\right) \Psi=0 .
\end{aligned}
$$

Das quais resultam:

$$
\begin{gathered}
\bar{\Psi}=\left(\bar{\Psi}_{L}+\bar{\Psi}_{R}\right)\left(\Psi_{L}+\Psi_{R}\right)=\bar{\Psi}_{R} \Psi_{L}+\bar{\Psi}_{L} \Psi_{R}, \\
\bar{\Psi} \gamma^{\mu} \partial_{\mu} \Psi=\left(\bar{\Psi}_{L}+\bar{\Psi}_{R}\right) \gamma^{\mu} \partial_{\mu}\left(\Psi_{L}+\Psi_{R}\right)=\bar{\Psi}_{L} \gamma^{\mu} \partial_{\mu} \Psi_{L}+\bar{\Psi}_{R} \gamma^{\mu} \partial_{\mu} \Psi_{R}, \\
\bar{\Psi} \gamma^{5} \partial_{y} \Psi=\left(\bar{\Psi}_{L}+\bar{\Psi}_{R}\right) \gamma^{5} \partial_{y}\left(\Psi_{L}+\Psi_{R}\right)=\bar{\Psi}_{L} \gamma^{5} \partial_{y} \Psi_{R}+\bar{\Psi}_{R} \gamma^{5} \partial_{y} \Psi_{L},
\end{gathered}
$$

Serão impostas condições de contorno periódicas:

$$
\Psi_{L, R}\left(x^{\mu}, \pi R\right)=\Psi_{L, R}\left(x^{\mu},-\pi R\right) .
$$

Seja também a expansão:

$$
e^{-4 \sigma} \partial_{y}\left(\bar{\Psi} \gamma^{5} \Psi\right)=\partial_{y}\left(e^{-4 \sigma} \bar{\Psi} \gamma^{5} \Psi\right)-\left(\partial_{y} e^{-4 \sigma}\right) \bar{\Psi} \gamma^{5} \Psi
$$

Segue que o primeiro termo do lado direito, quando integrado em $d y$, anulase, pois resulta na diferença entre $\left(e^{-4 \sigma} \bar{\Psi} \gamma^{5} \Psi\right)(\pi R)$ e $\left(e^{-4 \sigma} \bar{\Psi} \gamma^{5} \Psi\right)(-\pi R)$, que são iguais, pelas condições de contorno periódicas.

A equação (3.54), se torna:

$$
\begin{array}{r}
S_{s}=\int d^{4} x \int d y\left(e^{-3 \sigma} i \bar{\Psi} \gamma^{\mu} \partial_{\mu} \Psi-e^{-4 \sigma} \bar{\Psi} \gamma^{5} \partial_{y} \Psi-\right. \\
-\frac{1}{2}\left(\partial_{y} e^{-4 \sigma}\right) \bar{\Psi} \gamma^{5} \Psi- \\
\left.-e^{-4 \sigma} m \operatorname{sgn}(y) \bar{\Psi} \Psi\right)
\end{array}
$$

A decomposição dos campos:

$$
\Psi=\Psi_{L}+\Psi_{R}
$$

com o uso também da propriedade:

$$
\bar{\Psi}_{L, R} \gamma^{5} \Psi_{R, L}= \pm \bar{\Psi}_{L, R} \Psi_{R, L},
$$


leva a ação à forma:

$$
\begin{array}{r}
S_{s}=\int d^{4} x \int d y\left\{e^{-3 \sigma}\left(\bar{\Psi}_{L} i \gamma^{\mu} \partial_{\mu} \Psi_{L}+\bar{\Psi}_{R} i \gamma^{\mu} \partial_{\mu} \Psi_{R}\right)-\right. \\
-e^{-4 \sigma} m \operatorname{sgn}(y)\left(\bar{\Psi}_{L} \Psi_{R}+\bar{\Psi}_{R} \Psi_{L}\right)-\frac{1}{2}\left[2 e^{-4 \sigma}\left(\bar{\Psi}_{L} \partial_{y} \Psi_{R}-\bar{\Psi}_{R} \partial_{y} \Psi_{L}\right)+\right. \\
\left.\left.+\left(\partial_{y} e^{-4 \sigma}\right)\left(\bar{\Psi}_{L} \Psi_{R}-\bar{\Psi}_{R} \Psi_{L}\right)\right]\right\}
\end{array}
$$

da qual chega-se a:

$$
\begin{array}{r}
S_{s}=\int d^{4} x \int d y\left\{e^{-3 \sigma}\left(\bar{\Psi}_{L} i \gamma^{\mu} \partial_{\mu} \Psi_{L}+\bar{\Psi}_{R} i \gamma^{\mu} \partial_{\mu} \Psi_{R}\right)-\right. \\
-e^{-4 \sigma} m \operatorname{sgn}(y)\left(\bar{\Psi}_{L} \Psi_{R}+\bar{\Psi}_{R} \Psi_{L}\right)-\frac{1}{2}\left[\bar{\Psi}_{L}\left(e^{-4 \sigma} \partial_{y}+\partial_{y} e^{-4 \sigma}\right) \Psi_{R}-\right. \\
\left.\left.-\bar{\Psi}_{R}\left(e^{-4 \sigma} \partial_{y}+\partial_{y} e^{-4 \sigma}\right) \Psi_{L}\right]\right\}
\end{array}
$$

Da última expressão para a ação, fica explícito que a lagrangeana será par sob transformações $y \rightarrow-y$, se $\Psi_{L}$ for par e $\Psi_{R}$ for ímpar, ou vice-versa.

Sendo a lagrangeana par, sob transformações $y \rightarrow-y$, restringe-se o intervalo de integração a $0 \leq y \leq \pi R$ ficando dobrado o valor da integral. Omitindo-se este fator 2, que poderia ser incluído no início e que é irrelevante para as equações de movimento, seja o Ansatz (decomposição KK):

$$
\Psi_{L, R}\left(x^{\mu}, y\right)=\frac{e^{2 \sigma}}{\sqrt{\pi R}} \sum_{n} \psi_{n}^{L, R}\left(x^{\mu}\right) \hat{f}_{n}^{L, R}(y),
$$

$\operatorname{com}\left\{\hat{f}_{n}^{L, R}(y)\right\}$ dois conjuntos completos e ortonormais para $y \in[0, \pi R]$. O que se busca é, após a integração pela dimensão adicional, que a ação adquira a forma da ação de Dirac para vários férmions:

$$
S_{D}=\sum_{n} \int d^{4} x\left[\bar{\psi}_{n}(x) i \gamma^{\mu} \partial_{\mu} \psi_{n}(x)-m_{n} \bar{\psi}_{n}(x) \psi_{n}(x)\right]
$$

Substituindo a decomposição (3.70) na ação (3.69) (com o intervalo de integração restrito e omitindo-se o fator 2 , como discutido acima) resulta: 


$$
\begin{array}{r}
S_{s}=\frac{1}{\pi R} \sum_{n} \int d^{4} x\left\{\psi_{n}^{\dagger L}(x) \gamma^{0} \sum_{m} i \gamma^{\mu} \partial_{\mu} \psi_{m}^{L}(x) \int d y e^{\sigma} \hat{f}_{n}^{L *}(y) \hat{f}_{m}^{L}(y)+\right. \\
+\psi_{n}^{\dagger R}(x) \gamma^{0} \sum_{m} i \gamma^{\mu} \partial_{\mu} \psi_{m}^{R}(x) \int d y e^{\sigma} \hat{f}_{n}^{R *}(y) \hat{f}_{m}^{R}(y)+ \\
+\left[\psi _ { n } ^ { \dagger R } ( x ) \gamma ^ { 0 } \sum _ { m } \psi _ { m } ^ { L } ( x ) \int d y \left(\frac{1}{2} e^{2 \sigma} \hat{f}_{n}^{R *}(y)\left(e^{-4 \sigma} \partial_{y}+\partial_{y} e^{-4 \sigma}\right) e^{2 \sigma} \hat{f}_{m}^{L}(y)-\right.\right. \\
\left.-m \hat{f}_{n}^{R *}(y) \hat{f}_{m}^{L}(y)\right)-\psi_{n}^{\dagger L}(x) \gamma^{0} \sum_{m} \psi_{m}^{R}(x) \int d y\left(\frac { 1 } { 2 } e ^ { 2 \sigma } \hat { f } _ { n } ^ { L * } ( y ) \left(e^{-4 \sigma} \partial_{y}+\right.\right. \\
\left.\left.\left.\left.+\partial_{y} e^{-4 \sigma}\right) e^{2 \sigma} \hat{f}_{m}^{R}(y)+m \hat{f}_{n}^{L *}(y) \hat{f}_{m}^{R}(y)\right)\right]\right\}
\end{array}
$$

Esta última, comparada com (3.71), mostra que, deve valer para a normalização:

$$
\frac{1}{\pi R} \int_{0}^{\pi R} d y e^{k y} \hat{f}_{n}^{L, R *}(y) \hat{f}_{m}^{L, R}(y)=\delta_{n m}
$$

Resulta também:

$$
\begin{array}{r}
\frac{1}{\pi R} \int d^{4} x \sum_{n, m} \int d y\left\{m\left[\psi_{n}^{\dagger L} \gamma^{0} \psi_{m}^{R} \hat{f}_{n}^{L *} \hat{f}_{m}^{R}+\psi_{n}^{\dagger R} \gamma^{0} \psi_{m}^{L} \hat{f}_{n}^{R *} \hat{f}_{m}^{L}\right]+\right. \\
+\frac{1}{2}\left[\psi_{n}^{\dagger L} \gamma^{0} e^{2 \sigma} \hat{f}_{n}^{L *}\left(e^{-4 \sigma} \partial_{y}+\partial_{y} e^{-4 \sigma}\right) \psi_{m}^{R} e^{2 \sigma} \hat{f}_{m}^{R}-\right. \\
\left.\left.-\psi_{n}^{\dagger R} \gamma^{0} e^{2 \sigma} \hat{f}_{n}^{R *}\left(e^{-4 \sigma} \partial_{y}+\partial_{y} e^{-4 \sigma}\right) \psi_{m}^{L} e^{2 \sigma} \hat{f}_{m}^{L}\right]\right\}=\int d^{4} x \sum_{n} m_{n} \bar{\psi}_{n} \psi_{n}
\end{array}
$$

que se torna:

$$
\begin{array}{r}
\int d^{4} x \sum_{n}\left\{\sum _ { m } \psi _ { n } ^ { \dagger L } \gamma ^ { 0 } \psi _ { m } ^ { R } \int d y \left[m \hat{f}_{n}^{L *} \hat{f}_{m}^{R}+\frac{1}{2} \hat{f}_{n}^{L *}\left(e^{-2 \sigma} \partial_{y} e^{2 \sigma}+\right.\right.\right. \\
\left.\left.+e^{2 \sigma} \partial_{y} e^{-2 \sigma}\right) \hat{f}_{m}^{R}-m_{m} e^{\sigma} \hat{f}_{n}^{L *} \hat{f}_{m}^{L}\right]+ \\
+\psi_{n}^{\dagger R} \gamma^{0} \psi_{m}^{L} \int d y\left[m \hat{f}_{n}^{R *} \hat{f}_{m}^{L}-\frac{1}{2} \hat{f}_{n}^{R *}\left(e^{-2 \sigma} \partial_{y} e^{2 \sigma}+e^{2 \sigma} \partial_{y} e^{-2 \sigma}\right) \hat{f}_{m}^{L}-\right. \\
\left.\left.-m_{m} e^{\sigma} \hat{f}_{n}^{R *} \hat{f}_{m}^{R}\right]\right\}=0 .
\end{array}
$$

Com a equação:

$$
\left(e^{-2 \sigma} \partial_{y} e^{2 \sigma}+e^{2 \sigma} \partial_{y} e^{-2 \sigma}\right) \hat{f}_{m}^{L, R}=2 \partial_{y} \hat{f}_{m}^{L, R}
$$


obtém-se:

$$
\left( \pm \partial_{y}-m\right) \hat{f}_{n}^{L, R}=-m_{n} e^{k y} \hat{f}_{n}^{R, L}
$$

Como $\Psi_{L}$ deve ser par e $\Psi_{R}$ ímpar, ou vice-versa, para que a lagrangeana seja par sob as transformações $y \rightarrow-y$, seguem as condições de contorno:

$$
\hat{f}_{m}^{L *}(0) \hat{f}_{n}^{R}(0)=\hat{f}_{m}^{L *}(\pi R) \hat{f}_{n}^{R}(\pi R)=0
$$

Seja:

$$
t=\epsilon e^{\sigma} \in[\epsilon, 1]
$$

onde

$$
\epsilon=e^{-\pi k R}
$$

O componente dependente da dimensão extra se transforma em:

$$
\hat{f}_{n}^{L, R}(y)=\alpha f_{n}^{L, R}(t)
$$

que, com a imposição da condição de normalização (3.73) leva a:

$$
\alpha=\sqrt{k \epsilon}
$$

e

$$
\frac{1}{\pi R} \int_{\epsilon}^{1} d t f_{m}^{L, R *}(t) f_{n}^{L, R}(t)=\delta_{m n}
$$

A expressão (3.77) torna-se:

$$
\left( \pm k t \partial_{t}-m\right) f_{n}^{L, R}(t)=-m_{n} \frac{t}{\epsilon} f_{n}^{R, L}(t)
$$

que as definições:

$$
\nu=\frac{m}{k}
$$

e

$$
x_{n}=\frac{m_{n}}{\epsilon k}=\left(\frac{m_{n}}{k}\right) e^{\pi k R}
$$

levam a:

$$
\left( \pm t \partial_{t}-\nu\right) f_{n}^{L, R}(t)=-x_{n} t f_{n}^{R, L}(t)
$$

e às condições de contorno:

$$
f_{m}^{L *}(\epsilon) f_{n}^{R}(\epsilon)=f_{m}^{L *}(1) f_{n}^{R}(1)=0 .
$$


Para os modos zero $m_{n}=0, n=0$, segue que $x_{n}=0$. A substituição na equação (3.87) resulta em:

$$
\left( \pm t \partial_{t}-\nu\right) f_{0}^{L, R}(t)=0
$$

que tem solução:

$$
f_{0}^{L, R}(t)=f_{0}^{L, R}(1) t^{ \pm \nu}
$$

Estas funções satisfazem as equações (3.88) se e somente se $f_{0}^{L}(1)=0$ ou $f_{0}^{R}(1)=0$, se um for nulo, o outro não se anula, mas um dos dois deve ser nulo. Isto significa que apenas $\Psi_{L}$ ou $\Psi_{R}$ tem modo zero, se um tiver, o outro tem modo zero nulo. Como o modo zero corresponde a partícula do Modelo Padrão, é recuperada a quiralidade dos férmions do MP.

Usando a normalização, resulta para o modo zero:

$$
\left|f_{0}^{L, R}(1)\right|^{2}=\pi R \frac{1 \pm 2 \nu}{1-e^{-\pi k R(1 \pm 2 \nu)}}
$$

que, finalmente, leva à solução:

$$
f_{0}^{L, R}(t)=\sqrt{\frac{\pi R(1 \pm 2 \nu)}{1-e^{-\pi k R(1 \pm 2 \nu)}}} t^{ \pm \nu}
$$

A substituição para $y$ resulta em:

$$
\hat{f}_{0}^{L, R}(y)=\sqrt{\frac{\pi k R e^{-\pi k R}(1 \pm 2 \nu)}{1-e^{-\pi k R(1 \pm 2 \nu)}}} e^{ \pm k \nu(y-\pi R)} .
$$

Para os outros modos, a substituição do lado direito da equação (3.87) no lado esquerdo, leva a:

$$
\left[t^{2} \partial_{t}^{2}+x_{n}^{2} t^{2}-\nu(\nu \mp 1)\right] f_{n}^{L, R}(t)=0 .
$$

Para:

$$
f_{n}^{L, R}(t)=\sqrt{t} g_{n}^{L, R}(t)
$$

resulta a equação:

$$
t^{2} \partial_{t}^{2} g_{n}^{L, R}+t \partial_{t} g_{n}^{L, R}+x_{n}^{2}\left[t^{2}-\left(\nu(\nu \mp 1)+\frac{1}{4}\right) \frac{1}{x_{n}^{2}}\right] g_{n}^{L, R}=0 .
$$

Com:

$$
z=x_{n} t
$$


e

$$
\hat{g}_{n}^{L, R}(z)=g_{n}^{L, R}\left(z / x_{n}\right)=g_{n}^{L, R}(t)
$$

segue a equação:

$$
z^{2} \partial_{z}^{2} \hat{g}_{n}^{L, R}+z \partial_{z} \hat{g}_{n}^{L, R}+\left(z^{2}-\mu_{L, R}^{2}\right) \hat{g}_{n}^{L, R}=0
$$

onde

$$
\mu_{L, R}^{2}=\nu(\nu \mp 1)+\frac{1}{4}
$$

que pode ser reconhecida como a equação de Bessel.

Para $\nu \neq 1 / 2+N$, com $N$ inteiro, vale:

$$
\hat{g}_{n}^{L, R}(z)=a_{n}^{L, R} \mathrm{~J}_{\mu_{L, R}}(z)+b_{n}^{L, R} \mathrm{~J}_{-\mu_{L, R}}(z),
$$

que leva a:

$$
f_{n}(t)^{L, R}(t)=\sqrt{t}\left(a_{n}^{L, R} \mathrm{~J}_{1 / 2 \mp \nu}\left(x_{n} t\right)+b_{n}^{L, R} \mathrm{~J}_{-1 / 2 \pm \nu}\left(x_{n} t\right)\right) .
$$

Entretanto $f_{n}^{L, R}$ não são independentes, sendo acoplados pela equação (3.77).

Utilizando as soluções acima na equação (3.77), resultam as soluções:

$$
\begin{aligned}
& f_{n}^{L}(t)=\sqrt{t}\left[a_{n}^{L} \mathrm{~J}_{1 / 2-\nu}\left(x_{n} t\right)+a_{n}^{R} \mathrm{~J}_{-1 / 2+\nu}\left(x_{n} t\right)\right], \\
& f_{n}^{R}(t)=\sqrt{t}\left[a_{n}^{R} \mathrm{~J}_{1 / 2+\nu}\left(x_{n} t\right)-a_{n}^{L} \mathrm{~J}_{-1 / 2-\nu}\left(x_{n} t\right)\right] .
\end{aligned}
$$

Por fim, para a construção do Modelo Padrão, resta a caracterização do campo vetorial.

\subsubsection{Campo Vetorial}

O cálculo será apresentado, por simplicidade, para o caso de um bóson vetorial que respeita a simetria U(1). A ação é dada por [5]:

$$
S_{v}=\int d^{4} x \int d y \frac{\sqrt{g}}{4 g_{5}^{2}} F_{M N} F^{M N},
$$

onde $g_{5}$ é a constante de acoplamento de gauge em 5 dimensões $^{1}$ e:

$$
F_{M N}=\partial_{M} V_{N}-\partial_{N} V_{M}
$$

\footnotetext{
${ }^{1}$ Sendo que a partir dela se obtém $g$, a constante de acoplamento de gauge em 4 dimensões, como será observado adiante.
} 
ou seja:

$$
S_{v}=\int d^{4} x \int d y \frac{\sqrt{g}}{4 g_{5}^{2}}\left(\partial_{M} V_{N}-\partial_{N} V_{M}\right)\left(\partial_{P} V_{Q}-\partial_{Q} V_{P}\right) g^{P M} g^{Q N} .
$$

A variação leva a:

$$
\begin{array}{r}
\delta S_{v}=\int d^{4} x \int d y \frac{\sqrt{g}}{4 g_{5}^{2}} \frac{\delta}{\delta \partial_{R} V_{S}}\left[\left(\partial_{M} V_{N}-\partial_{N} V_{M}\right)\left(\partial_{P} V_{Q}-\partial_{Q} V_{P}\right)\right] \\
. g^{P M} g^{Q N} \delta \partial_{R} V_{S}
\end{array}
$$

que se torna:

$$
\begin{array}{r}
\delta S_{v}=\int d^{4} x \int d y \frac{\sqrt{g}}{4 g_{5}^{2}}\left[\left(g^{P R} g^{Q S}-g^{P S} g^{Q R}\right) F_{P Q}+\right. \\
\left.+F_{M N}\left(g^{R M} g^{S N}-g^{S M} g^{R N}\right)\right] \partial_{R} \delta V_{S}
\end{array}
$$

Aplicando a regra da cadeia para derivadas:

$$
\begin{aligned}
\delta S_{v}=\int d^{4} x \int d y & \left\{\partial _ { R } \left[\frac { \sqrt { g } } { 4 g _ { 5 } ^ { 2 } } \left(\left(g^{P R} g^{Q S}-g^{P S} g^{Q R}\right) F_{P Q}+\right.\right.\right. \\
& \left.\left.+F_{M N}\left(g^{R M} g^{S N}-g^{S M} g^{R N}\right)\right) \delta V_{S}\right]- \\
- & \left(\partial _ { R } \left[\frac { \sqrt { g } } { 4 g _ { 5 } ^ { 2 } } \left(\left(g^{P R} g^{Q S}-g^{P S} g^{Q R}\right) F_{P Q}+\right.\right.\right. \\
+ & \left.\left.\left.\left.F_{M N}\left(g^{R M} g^{S N}-g^{S M} g^{R N}\right)\right)\right]\right) \delta V_{S}\right\} .
\end{aligned}
$$

Da identidade:

$$
\left(g^{P R} g^{Q S}-g^{P S} g^{Q R}\right) F_{P Q}=2 g^{R P} g^{S Q} F_{P Q}
$$

segue que:

$$
\begin{aligned}
\delta S_{v}=\int d^{4} x \int & d y\left\{\partial_{R}\left[\frac{\sqrt{g}}{g_{5}^{2}} g^{R P} g^{Q S} F_{P Q} \delta V_{S}\right]-\right. \\
- & {\left.\left[\partial_{R}\left(\frac{\sqrt{g}}{g_{5}^{2}} g^{R P} g^{Q S} F_{P Q}\right)\right] \delta V_{S}\right\} . }
\end{aligned}
$$

Integrando-se o primeiro termo do lado direito, com o uso do teorema de Gauss e supondo campos que se anulem suficientemente depressa no infinito, 
resulta:

$$
\begin{gathered}
\delta S_{v}=\left.\int d^{4} x\left(\frac{\sqrt{g}}{g_{5}^{2}} g^{5 P} g^{Q S} F_{P Q} \delta V_{S}\right)\right|_{y=0} ^{y=\pi R}- \\
-\int d^{4} x \int d y\left[\partial_{R}\left(\frac{\sqrt{g}}{g_{5}^{2}} g^{R P} g^{Q S} F_{P Q}\right)\right] \delta V_{S}
\end{gathered}
$$

que leva à equação de movimento:

$$
\partial_{R}\left(\sqrt{g} g^{R P} g^{Q S} F_{P Q}\right)=0 .
$$

Escolhendo um gauge onde:

$$
V_{5}=0
$$

com o vínculo:

$$
\partial_{\mu} V^{\mu}=0
$$

segue a equação de movimento:

$$
\eta^{\mu \nu} \partial_{\mu} \partial_{\nu} V^{\lambda}-\partial_{y}\left(e^{-2 \sigma} \partial_{y} V^{\lambda}\right)=0 .
$$

A decomposição de Kaluza-Klein é:

$$
V^{\lambda}=\frac{1}{\sqrt{\pi R}} \sum_{n} V^{\lambda(n)}\left(x^{\mu}\right) f_{n}(y)
$$

com condição de normalização:

$$
\frac{1}{\pi R} \int_{0}^{\pi R} d y f_{n}(y) f_{m}(y)=\delta_{m n}
$$

Inserindo a decomposição de KK na equação (3.117) resulta:

$$
\sum_{n}\left[\left(\partial_{\mu} \partial^{\mu} V^{\lambda(n)}\right) f_{n}-V^{\lambda(n)} \partial_{y}\left(e^{-2 k y} \partial_{y} f_{n}\right)\right]=0
$$

Da imposição da equação de movimento para vetores massivos:

$$
\partial_{\mu} \partial^{\mu} V^{\lambda(n)}+m_{n}^{2} V^{\lambda(n)}=0,
$$

segue que:

$$
m_{n}^{2} f_{n}+\partial_{y}\left(e^{-2 k y} \partial_{y} f_{n}\right)=0 .
$$


Para o modo zero, $m_{n}=0$ e $n=0$ :

$$
e^{-2 k y} \partial_{y} f_{0}=C^{\prime}
$$

ou seja

$$
f_{0}=A+B e^{2 k y}
$$

O primeiro termo do lado direito de (3.113):

$$
\left.\int d^{4} x\left(\frac{\sqrt{g}}{g_{5}^{2}} g^{5 P} g^{Q S} F_{P Q} \delta V_{S}\right)\right|_{y=0} ^{y=\pi R}
$$

determina as condições de contorno. No caso de campo par, sob a transformação $y \rightarrow-y$, como $F_{5 \lambda}=\partial_{y} V_{\lambda}$, resulta que:

$$
\left.\frac{d}{d y} f_{n}\right|_{y=0, \pi R}=0
$$

para campos ímpares deve valer:

$$
\left.f_{n}(y)\right|_{y=0, \pi R}=0
$$

Então, no caso de campo par, o modo zero se torna:

$$
f_{0}=1
$$

considerando-se a normalização, equação (3.119). Para campo ímpar, não há modo zero. Substituindo

$$
f_{n}=e^{k y} g_{n}
$$

obtém-se:

$$
m_{n}^{2} e^{k y} g_{n}-k^{2} e^{-k y} g_{n}+e^{-k y} \partial_{y}^{2} g_{n}=0,
$$

que, com as definições:

$$
z=\frac{m_{n}}{k} e^{k y}
$$

$\mathrm{e}$

$$
g_{n}(y)=h_{n}(z)
$$

torna-se:

$$
z^{2} \partial_{z}^{2} h_{n}+z \partial_{z} h_{n}+\left(z^{2}-1\right) h_{n}=0,
$$

que é a equação de Bessel. 
Impondo a normalização, equação (3.119) e retornando a $f$, resulta:

$$
f_{n}(y)=\frac{e^{k y}}{N_{n}}\left[\mathrm{~J}_{1}\left(\frac{m_{n}}{k} e^{k y}\right)+b_{1} \mathrm{Y}_{1}\left(\frac{m_{n}}{k} e^{k y}\right)\right]
$$

com:

$$
N_{n}^{2}=\frac{1}{\pi R} \int_{0}^{\pi R} d y e^{2 k y}\left[\mathrm{~J}_{1}\left(\frac{m_{n}}{k} e^{k y}\right)+b_{1} \mathrm{Y}_{1}\left(\frac{m_{n}}{k} e^{k y}\right)\right]^{2} .
$$

Da equação (3.125), para campo par (eq. (3.126)) segue que:

$$
b_{1}=-\frac{\mathrm{J}_{1}\left(\frac{m_{n}}{k}\right)+\frac{m_{n}}{k} \mathrm{~J}_{1}^{\prime}\left(\frac{m_{n}}{k}\right)}{\mathrm{Y}_{1}\left(\frac{m_{n}}{k}\right)+\frac{m_{n}}{k} \mathrm{Y}_{1}^{\prime}\left(\frac{m_{n}}{k}\right)}
$$

com:

$$
b_{1}\left(m_{n}\right)=b_{1}\left(m_{n} e^{\pi k R}\right)
$$

Essas duas condições determinam $b_{1}$ e $m_{n}$. Nota-se que, para o limite $m_{n}<<k$ e $k R>>1$, vale a aproximação:

$$
m_{n} \approx\left(n-\frac{1}{4}\right) \pi k e^{-\pi k R}
$$

para $n>0$.

Para campo ímpar, a equação (3.127) leva a:

$$
b_{1}\left(m_{n}\right)=-\frac{\mathrm{J}_{1}\left(\frac{m_{n}}{k}\right)}{\mathrm{Y}_{1}\left(\frac{m_{n}}{k}\right)},
$$

onde vale novamente a equação (3.137). A aproximação para a massa, nesse caso, torna-se:

$$
m_{n} \approx\left(n+\frac{1}{4}\right) \pi k e^{-\pi k R}
$$

Deve ser ressaltado que o fato de um campo (vetorial, neste caso) ser par ou ímpar, pela transformação $y \rightarrow-y$, decorre dos acoplamentos do campo, de tal forma que a lagrangeana resulte par.

\subsection{Solução ao Problema da Hierarquia}

Para que o problema da hierarquia seja resolvido, isto é, para que haja uma explicação para a diferença entre a massa de Planck e a escala eletrofraca 
$(\sim 1 \mathrm{TeV})$ é suficiente, neste espaço, localizar o campo de Higgs próximo à brana TeV, enquanto os demais campos do Modelo Padrão podem se propagar na quinta dimensão. Para verificar, seja o caso limite onde o campo está localizado na brana TeV; a ação do campo de Higgs torna-se:

$$
\int d^{4} x \int d y \sqrt{g} \delta(y-\pi R)\left[g^{\mu \nu} \partial_{\mu} \widetilde{H}^{\dagger} \partial_{\nu} \widetilde{H}-\frac{\lambda}{4}\left(\widetilde{H}^{\dagger} \widetilde{H}-v_{0}^{2}\right)^{2}\right]+\ldots
$$

Da equação 3.7:

$$
g_{\mu \nu}=e^{-2 \sigma} \eta_{\mu \nu}
$$

segue que:

$$
\sqrt{g}=e^{-4 k y}
$$

que, substituída na equação 3.141 leva, após a integração pela dimensão adicional, a:

$$
S=\int d^{4} x\left[e^{-2 \pi k R} \partial_{\mu} \widetilde{H}^{\dagger} \partial^{\mu} \widetilde{H}-\frac{\lambda}{4}\left(\widetilde{H}^{\dagger} \widetilde{H}-v_{0}\right)^{2} e^{-4 \pi k R}\right]+\ldots
$$

Por fim, definindo:

$$
H=e^{-\pi k R} \widetilde{H}
$$

e

$$
v=e^{-\pi k R} v_{0}
$$

percebe-se que:

$$
\pi k R=\ln \left(\frac{v_{0}}{v}\right)
$$

Assim, para $v_{0}=M_{P}$, ou seja, se o valor esperado de vácuo do campo de Higgs, em 5-dimensões, for a massa de Planck, para que o valor observado em 4-dimensões seja $v=246 \mathrm{GeV}$, deve valer, para a dimensão extra, $k R \approx 12$. O problema da hierarquia fica então resolvido, uma vez que a diferença entre a escala da massa de Planck e a escala eletrofraca $(246 \mathrm{GeV})$ fica explicada pela curvatura do espaço.

Com o problema da hierarquia resolvido e uma vez que estão caracterizados os campos na teoria com uma dimensão extra, estes devem ser utilizados para a obtenção do Modelo Padrão, bem como para a construção de modelos que o estendam. 


\section{Capítulo 4}

\section{Troca de Sabor por Correntes Neutras}

Foi observado no capítulo anterior que, com o uso de teorias com uma dimensão adicional curva (WED), resolve-se o problema da hierarquia do Modelo Padrão, com o bóson de Higgs próximo à brana $\mathrm{TeV}$. $\mathrm{O}$ próximo passo é obter a teoria completa que contém o Modelo Padrão como limite a baixas energias, com os modos zero dos campos representando os campos do MP e respeitando as mesmas simetrias. Para tanto, é necessário determinar as constantes características de cada partícula que se pretenda introduzir, o que permitirá resolver também o problema da hierarquia das massas.

Como o bóson de Higgs ou um condensado de férmions que o substitua deve estar localizado próximo à brana $\mathrm{TeV}$, ficará associada a massa de cada partícula a sua localização na quinta dimensão, devido à forma particular de cada função de onda 5-dimensional dos modos zero. Isto porque os acoplamentos de Yukawa em 4 dimensões resultam de integrais, pela quinta dimensão, do produto das funções de onda do Higgs (ou do condensado) e do campo em particular. Assim, quanto maior a superposição da função de onda de qualquer partícula do Modelo Padrão com a função do bóson de Higgs, maior será sua massa. Desta maneira, quanto mais massivo o férmion, mais próxima da brana $\mathrm{TeV}$ é sua localização.

Uma vez caracterizado o modelo que contém o Modelo Padrão como limite a baixas energias, serão observadas interações fortes com o primeiro modo 
de Kaluza-Klein do glúon. Este bóson possui, como será observado, função de onda localizada próxima à brana $\mathrm{TeV}$ e, como os acoplamentos com os férmions dependem de suas massas, este bóson fornece um meio para que haja interações fortes a ponto de haver condensação de férmions, em particular, através de acoplamentos com os quarks mais pesados. É esperado que os quarks mais massivos possam condensar através de interação com o primeiro modo KK do glúon pois, como este modo do bóson e os quarks mais pesados estão próximos à brana TeV (os quarks são massivos e a função de onda do bóson está localizada próxima à brana $\mathrm{TeV}$ ), grande é a superposição de suas funções de onda 5-dimensionais, resultando em grandes acoplamentos, que podem ser tão intensos a ponto de se tornarem supercríticos, resultando na condensação.

A condensação deve ocorrer na escala $\mathrm{TeV}$, de forma que o problema da hierarquia seja evitado. Mas, para tanto, é necessário introduzir quarks ainda mais massivos do que o quark top, ou seja, deve estar presente uma quarta geração de férmions ainda não detectada. Para a condensação ocorrer na escala TeV é preciso que o quark que condensa tenha massa aproximadamente entre 500 e $600 \mathrm{GeV}$ [8].

Outra característica do primeiro modo KK do glúon é a troca de sabor dos quarks, mesmo sendo esse bóson de gauge uma partícula neutra. Esta é a principal e, possivelmente, a única característica que permite o exame experimental de uma teoria com a quebra dinâmica da simetria eletrofraca gerada por um condensado de férmions [9] e dada a sua importância, será estudada fenomenologicamente.

Sabe-se que os experimentos em física de partículas são cada vez mais precisos e novos efeitos sugeridos por modelos teóricos podem violar os resultados do MP apenas no limite de incerteza dos experimentos conhecidos, ou a energias nas quais o Modelo Padrão não foi testado até então, caso de experimentos ainda não realizados. Ou seja, os resultados experimentais impõem vínculos à teoria. Qualquer extensão do Modelo Padrão que se pretenda validar, deve obedecer a tais vínculos.

A troca de sabor por correntes neutras a nível árvore não viola vínculos experimentais, pois a localização dos férmions leves (que impõem os vínculos mais restritivos) é próxima à brana UV (Planck), o que implica em acoplamentos fracos com o primeiro modo de Kaluza-Klein do glúon, prin- 
cipal responsável pela troca de sabor a nível árvore. Para os férmions mais pesados, existem simetrias (globais ou de gauge) que podem ser impostas ao modelo e que inibem a violação de vínculos experimentais, principalmente originários de $B \bar{B}$ mixing [30,31].

A seguir, são apresentados o modelo e resultados para a determinação das constantes 5-D para os quarks do MP e para a quarta geração de quarks, resultados que respeitam vínculos do Modelo Padrão, bem como resultados que violam o MP em energias ainda não estudadas, mas que estarão disponíveis no LHC (Large Hadron Collider) do CERN. São determinados, a partir da obtenção das constantes dos quarks, os acoplamentos entre o primeiro modo de Kaluza-Klein do glúon e os quarks, bem como as misturas entre os quarks pela extensão da matriz CKM.

\subsection{Extensão do Grupo de Gauge}

A teoria inclui campos que se propagam na dimensão extra e, portanto, dão origem a modos de Kaluza-Klein. Como a teoria deve se reduzir ao Modelo Padrão, a baixas energias, os modos zero de seus campos devem ser idênticos aos campos do MP, respeitando exatamente as mesmas simetrias. Por outro lado, devem ser analisadas as consequências que o fato de se utilizar um espaço 5-D curvo (com os outros modos KK) tem sobre os experimentos, em particular o efeito sobre os parâmetros vinculados do Modelo Padrão.

Do capítulo anterior, sabe-se que há campos em 5-D cujos modos zero representam escalares, vetores e férmions quirais em 4-D. Desta forma, podese reconstruir o Modelo Padrão como uma teoria efetiva (a baixas energias) de uma teoria 5-D. O que é necessário então é determinar quais simetrias devem ser respeitadas pelos campos em 5 dimensões e qual é a alteração nos parâmetros vinculados do MP. Nota-se, em particular, que os férmions quirais são apenas os modos zero dos campos espinoriais, os outros modos não apresentam quiralidade.

O grupo de gauge do Modelo Padrão é, como visto anteriormente,

$$
S U(3)_{c} \times S U(2)_{L} \times U(1)_{Y}
$$

e é este grupo de simetrias que deve ser respeitado pelos modos zero dos 
campos da teoria 5-D. Mas os modos de Kaluza-Klein introduzem novos efeitos potencialmente perigosos para a teoria, pois poderiam violar medições muito precisas das interações eletrofracas, tais quais os parâmetros S e T de Peskin-Takeuchi [32].

Para estabelecer a teoria, deve ser feita a escolha de como inserir os campos, sendo que os parâmetros S e T têm papel fundamental nesta escolha. A solução do problema da hierarquia requer apenas que o bóson de Higgs esteja localizado próximo à brana $\mathrm{TeV}$.

Caso os campos de gauge e os campos fermiônicos possam se propagar no bulk (os campos possuem dependência na coordenada adicional $y$ diferente de um impulso $\delta\left(y-y_{0}\right)$ ), a teoria deixa de apresentar problemas graves com o parâmetro S [33], mas ainda possui contribuições excessivas ao parâmetro T, com o grupo de gauge usual do Modelo Padrão.

O problema é resolvido pela imposição de simetria de gauge adicional (chamada custodial) com campos que se propagam no bulk [33]. Esta simetria adicional eleva o grupo de gauge do modelo, em 5-D, para:

$$
S U(3)_{c} \times S U(2)_{L} \times S U(2)_{R} \times U(1)_{X}
$$

onde, para recuperar o grupo do Modelo Padrão, o grupo $S U(2)_{R}$ é quebrado a $U(1)_{R}$ por condições de contorno na brana de Planck e o grupo resultante é quebrado espontaneamente na brana de Planck $U(1)_{R} \times U(1)_{X} \rightarrow U(1)_{Y}$, recuperando-se o grupo de hipercarga.

Os detalhes das possíveis representações fermiônicas podem variar, não sendo crucial neste ponto a escolha de uma representação particular. A título de exemplo, a referência [33] introduz uma representação onde os férmions de mão direita passam a fazer parte de dubletos com outros férmions de mão direita, mas que não possuem modo zero e portanto não foram detectados experimentalmente.

Pode ser observado, também na referência [33], que o parâmetro S não apresenta problemas nesta teoria, para férmions leves localizados próximos à brana de Planck, se as massas dos primeiros modos de Kaluza-Klein dos bósons de gauge forem $O(\mathrm{TeV})$. O parâmetro $\mathrm{T}$ também não apresenta problemas neste caso, para um determinado intervalo de valores possíveis para a massa do bóson de Higgs. O modelo foi construído para evitar as enormes contribuições ao parâmetro $\mathrm{T}$ vindas da mistura de $Z^{(0)}$ (o modo 
zero do campo Z, que representa o campo do bóson $\mathrm{Z}$ do MP) com os outros modos de Kaluza-Klein do próprio campo $\left(Z^{(n)}\right)$, contribuições estas que estariam presentes caso a simetria $S U(2)_{R}$ não fosse incluída. A esta última simetria está associado um campo $Z^{\prime}$ que não possui modo zero, mas cujos modos KK se misturam com o $Z^{(0)}$ de tal forma que estas misturas cancelam, aproximadamente, as misturas de $Z^{(0)}$ com os modos $Z^{(n)}$.

Apesar de a extensão do grupo de gauge representar complicações, em particular os férmions de mão direita fazem parte de dubletos, as constantes características de suas funções de onda em 5-D, que determinam suas localizações, são determinadas para o dubleto, mas são as mesmas para o férmion de mão direita correspondente. Assim, para caracterizar os férmions do Modelo Padrão basta determinar as constantes das funções de onda dos dubletos.

Um método distinto para se evitar problemas com os parâmetros de precisão eletrofracos, atualmente em estudo, consiste em uma modificação na métrica 3.3, de tal forma que o problema da hierarquia ainda seja resolvido, mas as contribuições aos parâmetros $\mathrm{S}$ e T sejam reduzidas, com a localização do bóson de Higgs próxima à brana TeV [34], porém não tanto quanto para a métrica de Randall-Sundrum. Neste método, não é introduzido o grupo de simetria $S U(2)_{R}$.

O problema da hierarquia também é resolvido caso o bóson de Higgs não seja elementar, mas sim composto, sendo na verdade um condensado de quarks. Entretanto, mesmo com o condensado, a simetria custodial deve estar presente, sendo a métrica dada pela equação (3.3). Esta é a abordagem adotada para esta teoria, onde os férmions que condensam são apenas os modos zero dos quarks do tipo up de uma quarta geração mais massiva.

Para implementação do mecanismo de condensação, será utilizada outra característica favorável da teoria, que são os acoplamentos não universais dos modos excitados dos bósons de gauge com os modos zero dos férmions. Como será observado, as funções de onda dos modos de Kaluza-Klein dos bósons localizam-se próximas à brana TeV. Assim, como cada férmion possui localização distinta na quinta dimensão, cada férmion possuirá acoplamentos distintos com os modos KK dos bósons e, quanto mais massivo for o férmion, mais intenso será seu acoplamento com estes modos KK. O acoplamento do quark mais massivo (tomado como o quark do tipo up da quarta geração) 
com o primeiro modo de Kaluza-Klein do glúon será mais intenso do que os acoplamentos deste bóson com os demais quarks, portanto, será este o quark que condensa. Poderia ocorrer a condensação de outros quarks, por exemplo o quark do tipo down da quarta geração, mas este caso não será aqui tratado.

\subsection{Modo KK do Glúon}

O modelo introduz novos acoplamentos, com os modos de Kaluza-Klein (KK) dos bósons de gauge. De particular interesse são os acoplamentos entre os quarks e o primeiro modo KK do glúon. Esses acoplamentos são interessantes pois, para os quarks mais pesados (tais quais o top e os quarks da quarta geração), são acoplamentos mais fortes do que o acoplamento com o glúon, como será observado. Além disso, esse modo excitado do glúon torna quirais as interações fortes e apresenta correntes neutras que trocam sabor.

Retomando as expansões em modos KK para os férmions:

$$
\Psi(x, y)=\frac{1}{\sqrt{\pi R}} \sum_{n} e^{2 k y} f_{n}(y) \psi^{(n)}(x)
$$

e para os bósons vetoriais:

$$
A_{\mu}^{a}(x, y)=\frac{1}{\sqrt{\pi R}} \sum_{n} \chi_{n}(y) A_{\mu}^{a(n)}(x),
$$

onde, o índice $a$ indica que a simetria respeitada pelo campo de gauge pode ser não-abeliana.

O termo de interação entre bóson e férmion, obtido da derivada covariante, é dado por:

$$
S_{f b}=-g_{5} \int d^{4} x \int_{0}^{\pi R} d y \sqrt{g} \bar{\Psi}(x, y) e^{k y} \gamma^{\mu} \mathbf{T}^{a} \Psi(x, y) A_{\mu}^{a}(x, y),
$$

com $\mathbf{T}^{a}$ os geradores do grupo de simetria respeitado. Desse termo se obtém, substituindo a expansão de KK para os campos, as constantes de acoplamento entre os modos $i$ dos férmions e os modos $n$ dos bósons:

$$
g_{i n}=\frac{g}{\pi R} \int_{0}^{\pi R} d y e^{k y}\left|f_{i}(y)\right|^{2} \chi_{n}(y),
$$


onde

$$
g=\frac{g_{5}}{\sqrt{\pi R}}
$$

levando à expansão:

$$
\begin{aligned}
S_{f b} & =-g_{00} \int d^{4} x \bar{\psi}^{(0)}(x) \gamma^{\mu} \mathbf{T}^{a} \psi^{(0)}(x) A_{\mu}^{a(0)}(x)- \\
& -g_{01} \int d^{4} x \bar{\psi}^{(0)}(x) \gamma^{\mu} \mathbf{T}^{a} \psi^{(0)}(x) A_{\mu}^{a(1)}(x)+\ldots
\end{aligned}
$$

da qual é interessante, para este trabalho, apenas os termos exibidos (os dois primeiros), pois são termos que relacionam os modos zero dos férmions com os primeiros modos KK dos bósons.

As funções de onda são, para os modos zero dos férmions, dadas pela equação (3.93):

$$
f_{0}^{L, R}(y)=\sqrt{\frac{\pi k R e^{-\pi k R}\left(1 \mp 2 c_{L, R}\right)}{1-e^{-\pi k R\left(1 \mp 2 c_{L, R}\right)}}} e^{\mp k c_{L, R}(y-\pi R)},
$$

sendo a constante $c$ relacionada ao parâmetro de massa 5-dimensional dos férmions, como observa-se no capítulo 3 e, pela equação acima, evidentemente determina a localização do férmions na quinta dimensão por determinar a forma da função de onda. Para $n \geq 1$, vale para os bósons:

$$
\chi_{n}(y)=\frac{e^{k y}}{N_{n}}\left[\mathrm{~J}_{1}\left(\frac{m_{n}}{k} e^{k y}\right)+b_{1} \mathrm{Y}_{1}\left(\frac{m_{n}}{k} e^{k y}\right)\right],
$$

com:

$$
N_{n}^{2}=\frac{1}{\pi R} \int_{0}^{\pi R} d y e^{2 k y}\left[\mathrm{~J}_{1}\left(\frac{m_{n}}{k} e^{k y}\right)+b_{1} \mathrm{Y}_{1}\left(\frac{m_{n}}{k} e^{k y}\right)\right]^{2}
$$

e

$$
b_{1}=-\frac{\mathrm{J}_{1}\left(\frac{m_{n}}{k}\right)+\frac{m_{n}}{k} \mathrm{~J}_{1}^{\prime}\left(\frac{m_{n}}{k}\right)}{\mathrm{Y}_{1}\left(\frac{m_{n}}{k}\right)+\frac{m_{n}}{k} \mathrm{Y}_{1}^{\prime}\left(\frac{m_{n}}{k}\right)},
$$

valendo também:

$$
b_{1}\left(m_{n}\right)=b_{1}\left(m_{n} e^{\pi k R}\right),
$$

pois da equação (4.5), fica claro que para a dada interação, o campo vetorial deve ser par sob as transformações $y \rightarrow-y$. Caso $n=0, \chi_{0}(y)=1$.

Segue que:

$$
g_{00}=g
$$


e

$$
\begin{array}{rl}
g_{01}=g \int_{0}^{\pi k R} d & y \frac{\left(1 \mp 2 c_{L, R}\right)}{\left(e^{\left(1 \mp 2 c_{L, R}\right) \pi k R}-1\right)} \frac{e^{2\left(1 \mp c_{L, R}\right) y}}{N_{1}} . \\
\cdot\left[\mathrm{J}_{1}\left(\frac{m_{n}}{k} e^{y}\right)+b_{1} \mathrm{Y}_{1}\left(\frac{m_{n}}{k} e^{y}\right)\right] .
\end{array}
$$

Para que o termo (4.5) acomode as interações eletrofracas, $\Psi(x, y)$ deve ser um dubleto de $\mathrm{SU}(2)_{L}$. Ou seja, na equação (4.9), nota-se que cada constante $c$ dos campos $f_{0}^{L}$ caracteriza o campo $L$ de cada dubleto de $\mathrm{SU}(2)_{L}$. A partícula propriamente dita, fica caracterizada por duas constantes: $c_{L}$ do dubleto do qual faz parte e $c_{R}$ do singleto, constante exclusiva para a partícula.

Comparando o a equação (4.5) com as interações da QCD, segue que $g_{00}$ é o próprio acoplamento da QCD e $g_{01}$ é o acoplamento do primeiro modo excitado de Kaluza-Klein (primeiro modo KK) do glúon, proporcional ao acoplamento da QCD, mas distinto para cada quark e mesmo para cada quiralidade do mesmo quark.

\subsection{Geração de Massas}

Como discutido anteriormente, será adotado $k R=12$, valendo, para a dimensão compactificada em orbifold, $0 \leq y \leq \pi R$, de tal forma que $M_{W} \sim$ $M_{P} e^{-\pi k R}$. Para que o modelo fique definido, é necessário obter as massas e misturas dos quarks corretamente, ou seja, com os valores determinados experimentalmente, antes de aplicar o modelo a cálculos de seções de choque por exemplo. Para tanto, é necessário determinar o mecanismo de geração de massa.

No mecanismo de geração de massas que será adotado, o bóson de Higgs surge da condensação do quark do tipo up de uma quarta geração, mais massiva do que as demais. Há, além das interações de gauge, interações por operadores de dimensão superior no bulk (ou seja, que não estão localizados apenas nas branas), que não são renormalizáveis, estando suprimidos por elevada escala de energias, em comparação à escala eletrofraca.

Como indicado na referência [8], interações induzidas por operadores de dimensão superior podem estar presentes. De particular interesse, o operador 
de quatro férmions:

$$
\mathcal{O}=\int d y \sqrt{g} \frac{C^{i j k l}}{M_{P}^{3}} \bar{\Psi}_{L}^{i}(x, y) \Psi_{R}^{j}(x, y) \bar{\Psi}_{R}^{k}(x, y) \Psi_{L}^{l}(x, y)
$$

(onde os índices $i, j, k, l$ indicam o sabor do férmion), dá origem ao operador dos modos zero de quatro férmions, em quatro dimensões (com a quinta dimensão integrada) [5]:

$$
\mathcal{O}_{4}=C^{i j k l} \frac{k}{M_{P}^{3}} \frac{\left(e^{\pi k R\left(4-c_{L}^{i}+c_{R}^{j}+c_{R}^{k}-c_{L}^{l}\right)}-1\right)}{\left(4-c_{L}^{i}+c_{R}^{j}+c_{R}^{k}-c_{L}^{l}\right)} \frac{\bar{\psi}_{L}^{i(0)}(x) \psi_{R}^{j(0)}(x) \bar{\psi}_{R}^{k(0)}(x) \psi_{L}^{l(0)}(x)}{N_{L}^{i} N_{R}^{j} N_{R}^{k} N_{L}^{l}}
$$

sendo $N_{L}^{i}$ as constantes de normalização dos modos zero dos férmions, obtidas da equação (3.93).

Como será observado, os acoplamentos mais fortes são aqueles do modo KK do glúon com os quarks da quarta geração, em particular com o quark (positivamente carregado) $u_{4}$, que é suposto mais massivo. Isto ocorre por causa da superposição das funções de onda 5-dimensionais do férmion e do modo KK do bóson; quanto mais massivo for o férmion, maior será esta superposição e, portanto, maior será o acoplamento.

Para energias abaixo da massa do modo KK do glúon, escala de energia definida para a teoria efetiva, o acoplamento efetivo para interações de quatro férmions, induzido pelo primeiro modo de Kaluza-Klein do glúon é, para o quark $u_{4}$ :

$$
\mathcal{O}_{4 g}=-\frac{g_{01}^{L} g_{01}^{R}}{M_{K K}^{2}}\left(\bar{u}_{4 L} \gamma_{\mu} \mathbf{T}^{a} u_{4 L}\right)\left(\bar{u}_{4 R} \gamma^{\mu} \mathbf{T}^{a} u_{4 R}\right),
$$

sendo $g_{01}^{L, R}$ o acoplamento entre o primeiro modo de Kaluza-Klein do glúon e o campo do quark $u_{4}$, componente $L$ ou $R$ e $\mathbf{T}^{a}$ representando os geradores de $S U(3)_{c}$. Este termo pode ser reescrito (mediante o re-arranjo de Fierz) como:

$$
\mathcal{O}_{4 g}=\frac{g_{01}^{L} g_{01}^{R}}{M_{K K}^{2}}\left(\bar{u}_{4 L}^{a} u_{4 R}^{a} \bar{u}_{4 R}^{b} u_{4 L}^{b}-\frac{1}{N_{c}} \bar{u}_{4 L}^{a} u_{4 R}^{b} \bar{u}_{4 R}^{b} u_{4 L}^{a}\right),
$$

sendo $a, b$ índices de $S U(3)_{c}$, o primeiro termo um singleto de cor, o segundo um octeto e suprimido por $1 / N_{c}$, onde $N_{c}$ é o número de cores. Assim, será considerado apenas o primeiro termo.

Então, para o quark $u_{4}$, considerando-se termos da equação (4.19) e da 
equação (4.17) [8] segue o acoplamento efetivo entre quatro férmions:

$$
g_{U}^{2} \equiv g_{01}^{L} g_{01}^{R}+\left(\frac{M_{K K}}{k e^{-\pi k R}}\right)^{2} C_{u u}^{4444}\left(\frac{k}{M_{P}}\right)^{3} \frac{\left(1-2 c_{L}^{4}\right)\left(1+2 c_{R}^{4}\right)}{2\left(2-c_{L}^{4}+c_{R}^{4}\right)} .
$$

Há um valor para $g_{U}$ acima do qual se forma um condensado:

$$
<\bar{u}_{4 L} u_{4 R}>\neq 0
$$

que leva à quebra da simetria eletrofraca.

Para obter o valor crítico, considera-se o modelo de Nambu-Jona-Lasinio (NJL) [35], modelo simplificado para a condensação de férmions e quebra dinâmica da simetria eletrofraca, tal qual o desenvolvimento de [21]. Iniciase com a lagrangeana [8]:

$$
\mathcal{L}_{\text {cond }}=\bar{u}_{4 L} i \gamma^{\mu} D_{\mu} u_{4 L}+\bar{u}_{4 R} i \gamma^{\mu} D_{\mu} u_{4 R}+\frac{g_{U}^{2}}{M_{K K}^{2}}\left(\bar{u}_{4 L} u_{4 R} \bar{u}_{4 R} u_{4 L}\right),
$$

que pode ser obtida da lagrangeana:

$$
\mathcal{L}_{\text {condH }}=\bar{u}_{4 L} i \gamma^{\mu} D_{\mu} u_{4 L}+\bar{u}_{4 R} i \gamma^{\mu} D_{\mu} u_{4 R}+g_{U} \bar{q}_{4 L} H u_{4 R}-M_{K K}^{2} H^{\dagger} H+\text { h.c. },
$$

mediante a eliminação por integração (processo de integrate-out) do campo $H$ (conforme referência [2]), onde $q_{4 L} \equiv\left(u_{4 L}, d_{4 L}\right)^{T}, H$ é um campo auxiliar que não se propaga, dubleto de $S U(2)_{L}$ e os termos cinéticos para $d_{4}$ foram omitidos.

Correções de ordem superior que envolvem loops de $u_{4}$, como, por exemplo, a correção da contribuição dada pelo diagrama da figura 4.1, a energias $\mu<M_{K K}$, levam ao surgimento de termo cinético para $H$ e a lagrangeana à forma:

$$
\begin{array}{r}
\mathcal{L}_{\text {cond } H}=Z_{u_{4 L}} \bar{u}_{4 L} i \gamma^{\mu} D_{\mu} u_{4 L}+Z_{u_{4 R}} \bar{u}_{4 R} i \gamma^{\mu} D_{\mu} u_{4 R}+\ldots+ \\
+Z_{g_{U}} g_{U} \bar{q}_{4 L} H u_{4 R}+\text { h.c. }+Z_{H}\left(D_{\mu} H\right)^{\dagger} D^{\mu} H-M_{H}^{2} H^{\dagger} H-\frac{\lambda}{2}\left(H^{\dagger} H\right)^{2}
\end{array}
$$

sendo os $Z_{i}$ 's resultantes das renormalizações. A contribuição mais relevante para $M_{H}$ leva a:

$$
M_{H}^{2}=M_{K K}^{2}\left(1-\frac{g_{U}^{2} N_{c}}{8 \pi^{2}}\right)+\ldots
$$




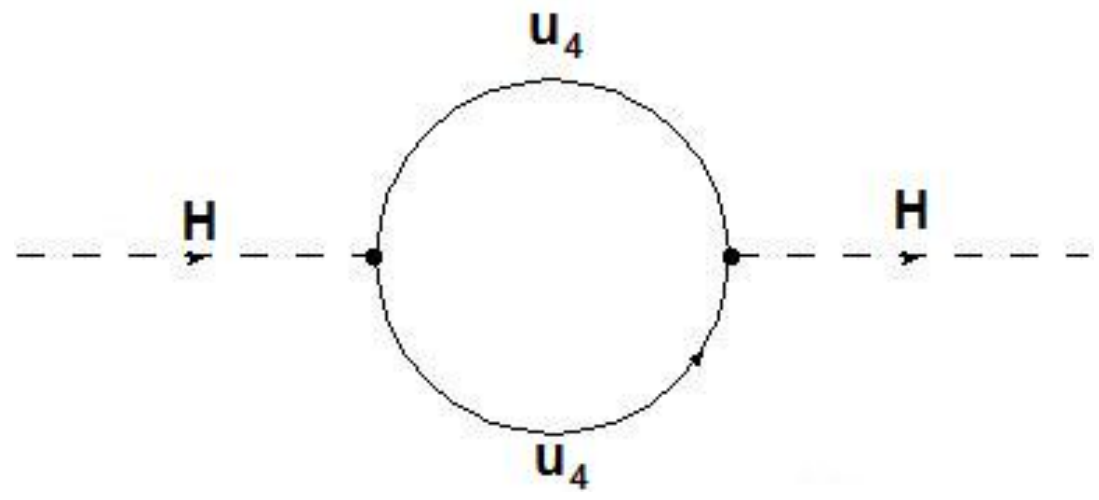

Figura 4.1: Contribuição quântica à massa do campo auxiliar $H$.

Fica claro, então, que o potencial efetivo para $H$ desenvolve um valor esperado de vácuo não trivial para:

$$
g_{U}^{2}>\frac{8 \pi^{2}}{N_{c}}
$$

No caso de condensação do quark $u_{4}$, os campos $u_{4 L}$ e $u_{4 R}$ condensam, o que faz com que o operador da equação (4.16) se torne um operador de 2 férmions, que gera massa a matriz de massa para todos os demais.

Assim sendo, as matrizes de massa podem ser colocadas na forma [36]:

$$
M_{Q}^{i j}=\lambda_{Q}^{i j} m_{1} f\left(c_{L}^{i}\right) f\left(-c_{R}^{j}\right) \alpha\left(c_{L}^{i},-c_{R}^{j}\right),
$$

onde

$$
f(c)=\sqrt{\frac{1-2 c}{1-x^{1-2 c}}},
$$

corresponde à constante de normalização dos modos zero dos quarks (lembrando que os modos zero dos férmions é que correspondem a férmions do MP) e:

$$
\begin{gathered}
x=e^{-\pi k R}, \\
\alpha\left(c_{i},-c_{j}\right)=\frac{f\left(c_{q}^{4}\right) f\left(-c_{u}^{4}\right)\left(1-x^{4-c_{q}^{4}+c_{u}^{4}-c_{i}+c_{j}}\right)}{\left(4-c_{q}^{4}+c_{u}^{4}-c_{i}+c_{j}\right)}, \\
m_{1}=\frac{<\bar{u}_{L}^{4(0)} u_{R}^{4(0)}>}{k^{2} x^{2}}\left(\frac{k}{M_{P}}\right)
\end{gathered}
$$


sendo, para a completa determinação do modelo a seguir, $m_{1}$ tratado como parâmetro a ser determinado para o ajuste das funções de onda dos modos zero. Para se evitar o problema da hierarquia das massas, deve valer $\left|\lambda_{Q}^{i j}\right| \sim$ $O(1)$.

A referência [36] traz ainda expressões correspondentes no caso de um bóson de Higgs fundamental. É mostrado [8] que quando a escala da teoria (a massa de modos de Kaluza-Klein do glúon, responsável pela condensação) é da ordem de poucos TeV, é necessário que as massas dos quarks que condensam estejam no intervalo $500 \sim 600 \mathrm{GeV}$. Além disso, para que sejam respeitados vínculos no parâmetro $\mathrm{T}$, as massas de $u_{4}$ e de $d_{4}$ devem satisfazer: $\left|m_{u_{4}}-m_{d_{4}}\right|<M_{W}$.

Então a condensação de $u_{4}$ gera as matrizes de massa para todos os quarks (na verdade para todos os férmions, mas enfatiza-se, nesta tese, o setor dos quarks), dependentes das constantes 5-dimensionais $\left(c_{q}\right)$ de cada quark. Para cálculos de seções de choque de processos, devem ser determinadas as constantes $c_{L, R}$ para se obterem os acoplamentos correspondentes. Isto é feito através da diagonalização das matrizes de massa, com o vínculo de se obter o setor conhecido no Modelo Padrão da matriz CKM e as corretas massas dos quarks.

\subsection{Parâmetros dos Quarks}

Com a inclusão da quarta geração, a lagrangeana para o termo de massas dos quarks é dada por:

$$
-\mathcal{L}_{\text {massa }}=\bar{U}_{L}^{\prime} \mathbf{M}_{U}^{\prime} U_{R}^{\prime}+\bar{D}_{L}^{\prime} \mathbf{M}_{D}^{\prime} D_{R}^{\prime}+\text { h.c. }
$$

onde

$$
\begin{aligned}
& U^{\prime T}=\left(u^{\prime}, c^{\prime}, t^{\prime}, u_{4}^{\prime}\right), \\
& D^{\prime T}=\left(d^{\prime}, s^{\prime}, b^{\prime}, d_{4}^{\prime}\right) .
\end{aligned}
$$

Os termos com linha, $u^{\prime}$ por exemplo, são campos do quark correspondente na base de interação, na qual se escrevem os termos que respeitam as simetrias de gauge na lagrangeana da teoria e as matrizes $\mathbf{M}^{\prime}$ não são diagonais. Tal qual no Modelo Padrão, as bases de interação e de massa (sem 
linha) estão relacionadas:

$$
\begin{aligned}
U_{L}^{\prime} & =\mathbf{S}_{L}^{u} U_{L}, \\
U_{R}^{\prime} & =\mathbf{S}_{R}^{u} U_{R}, \\
D_{L}^{\prime} & =\mathbf{S}_{L}^{d} D_{L}, \\
D_{R}^{\prime} & =\mathbf{S}_{R}^{d} D_{R},
\end{aligned}
$$

com as matrizes $\mathbf{S}$ unitárias, relação que induz as diagonalizações bi-unitárias das matrizes de massa:

$$
\begin{aligned}
& \mathbf{M}_{U}^{\prime}=\mathbf{S}_{L}^{u} \mathbf{M}_{U} \mathbf{S}_{R}^{u \dagger}, \\
& \mathbf{M}_{D}^{\prime}=\mathbf{S}_{L}^{d} \mathbf{M}_{D} \mathbf{S}_{R}^{d \dagger}
\end{aligned}
$$

onde as matrizes $\mathbf{M}$ são diagonais, por exemplo:

$$
\mathbf{M}_{D}=\left(\begin{array}{cccc}
m_{d} & 0 & 0 & 0 \\
0 & m_{s} & 0 & 0 \\
0 & 0 & m_{b} & 0 \\
0 & 0 & 0 & m_{d 4}
\end{array}\right)
$$

de forma que a lagrangeana (4.32) se reescreve como:

$$
-\mathcal{L}_{\text {massa }}=\bar{U}_{L} \mathbf{M}_{U} U_{R}+\bar{D}_{L} \mathbf{M}_{D} D_{R}+\text { h.c. }
$$

Para a matriz de massa diagonal (que é real), ao quadrado, vale:

$$
\mathbf{M}_{Q}^{2}=\mathbf{M}_{Q}^{\dagger} \mathbf{M}_{Q}=\mathbf{M}_{Q} \mathbf{M}_{Q}^{\dagger}
$$

e da primeira igualdade segue que:

$$
\mathbf{M}_{Q} \mathbf{M}_{Q}^{\dagger}=\left(\mathbf{S}_{L}^{q \dagger} \mathbf{M}_{Q}^{\prime} \mathbf{S}_{R}^{q}\right)\left(\mathbf{S}_{R}^{q \dagger} \mathbf{M}_{Q}^{\prime \dagger} \mathbf{S}_{L}^{q}\right)=\mathbf{S}_{L}^{q \dagger} \mathbf{M}_{Q}^{\prime} \mathbf{M}_{Q}^{\prime \dagger} \mathbf{S}_{L}^{q},
$$

usando a uniteriedade de $\mathbf{S}_{R}$. Segue também que:

$$
\mathbf{M}_{Q}^{\dagger} \mathbf{M}_{Q}=\left(\mathbf{S}_{R}^{q \dagger} \mathbf{M}_{Q}^{\prime \dagger} \mathbf{S}_{L}^{q}\right)\left(\mathbf{S}_{L}^{q \dagger} \mathbf{M}_{Q}^{\prime} \mathbf{S}_{R}^{q}\right)=\mathbf{S}_{R}^{q \dagger} \mathbf{M}_{Q}^{\prime \dagger} \mathbf{M}_{Q}^{\prime} \mathbf{S}_{R}^{q}
$$

Ou seja, as matrizes $\mathbf{S}$, apesar de não diagonalizarem $\mathbf{M}^{\prime}$, diagonalizam $\mathbf{M}^{\prime 2}$. Então, da equação (4.27) determinam-se os parâmetros $c$ numericamente, tais que os auto-valores de $M^{2}$ sejam as massas ao quadrado dos quarks. Mesmo que os parâmetros não sejam determinados univocamente, mas haja um espaço de parâmetros que satisfaçam os vínculos de massa 
e mistura, há pequena variação significativa nos novos acoplamentos, para parâmetros dentro desse espaço.

As massas dos quarks são determinadas, indiretamente, por experimentos. A referência [1] apresenta os resultados para as três gerações do MP, com as incertezas:

Tabela 1 - Massas dos Quarks

\begin{tabular}{|c|c|}
\hline Sabor & Massa $(\mathrm{MeV})$ \\
\hline $\mathrm{u}$ & 1,5 a 3,3 \\
$\mathrm{~d}$ & 3,5 a 6,0 \\
$\mathrm{c}$ & $1,27_{-0,11}^{+0,07} \cdot 10^{3}$ \\
$\mathrm{~s}$ & $104_{-34}^{+26}$ \\
$\mathrm{t}$ & $171,2(21) \cdot 10^{3}$ \\
$\mathrm{~b}$ & $4,20_{-0,07}^{+0,17} \cdot 10^{3}$ \\
\hline
\end{tabular}

Também valem os vínculos:

$$
\begin{gathered}
m_{u} / m_{d}=0,35 \text { a } 0,60 \\
m_{s} / m_{d}=17 \text { a } 22 \\
\left(m_{s}-\left(m_{u}+m_{d}\right) / 2\right) /\left(m_{d}-m_{u}\right)=30 a 50 .
\end{gathered}
$$

Uma vez que, pela variação numérica dos parâmetros $c$ e $\lambda^{i j}$ (parâmetros da matriz de massa, equação 4.27), foram obtidos os valores corretos de massa ao quadrado para os quarks, como auto-valores de $\mathbf{M}^{2}$, determina-se a matriz que diagonaliza $\mathbf{M}^{2}$ obtendo-se os seus auto-vetores. Para os quarks do tipo $u$ e do tipo $d$, estas matrizes levam a $\mathbf{S}_{L}^{u}$ e $\mathbf{S}_{L}^{d}$, o que possibilita a determinação da extensão 4x4 da matriz CKM, dada por:

$$
\mathbf{V}=\mathbf{S}_{L}^{u \dagger} \mathbf{S}_{L}^{d}
$$

Antes da determinação de todos os parâmetros, é comparada essa extensão da matriz CKM com os valores experimentais da matriz CKM. Após essa comparação, ficam determinados os parâmetros que caracterizam os férmions e que servirão para a determinação de novos acoplamentos que levam a novas interações e seções de choque, objetos de estudo experimental. 
Para o Modelo Padrão, com apenas três gerações, os valores experimentais são dados por:

$$
\mathbf{V}=\left(\begin{array}{ccc}
0,97418(27) & 0,2255(19) & 0,00393(36) \\
0,230(11) & 1,04(6) & 0,0412(11) \\
V_{t d} & V_{t s} & V_{t b}
\end{array}\right)
$$

$\operatorname{com}\left|V_{t d} / V_{t s}\right|=0,21(4)$ e $\left|V_{t b}\right|>0,74$.

Estão sob estudo dois casos representativos da teoria, o primeiro no qual a massa do quark do tipo up vale $m_{u_{4}}=500 \mathrm{GeV}$ e $m_{d_{4}}=450 \mathrm{GeV}$ e o segundo caso, no qual $m_{u_{4}}=600 \mathrm{GeV}$ e $m_{d_{4}}=550 \mathrm{GeV}$. Estes valores para as massas são representativos do intervalo de massas obtido de [8] e as diferenças de massas entre $u_{4}$ e $d_{4}$ são menores que $M_{W}$, para evitar contribuições excessivas ao parâmetro T e tendo como consequência a inibição do processo $u_{4} \rightarrow d_{4} W^{+}$. Então, serão determinados dois conjuntos (um para cada caso mencionado acima) de parâmetros, que são as constantes $c$ que estão presentes na equação equação $(4.28)^{1}$ :

$$
f(c)=\sqrt{\frac{1-2 c}{1-x^{1-2 c}}} .
$$

\footnotetext{
${ }^{1}$ As demais constantes presentes na equação $(4.27)\left(\lambda_{i j} \times m_{1}\right)$ são todas da mesma ordem, para que se resolva o problema da hierarquia das massas. Sua determinação é essencial mas a importância de se conhecer seus valores é indireta, uma vez que só aparecem combinadas para formar observáveis relevantes como as corretas massas dos quarks ou a matriz CKM, sendo, portanto, estes valores derivados mostrados em lugar dessas constantes.
} 
Para o primeiro caso resultam:

Tabela 2 - Caso 1: $m_{u_{4}}=500 \mathrm{GeV}$
\begin{tabular}{c|c|}
\hline Campo & Constante c \\
\hline$u_{4 L}$ e $d_{4 L}$ & $c_{q_{4}}=0,02$ \\
$u_{4 R}$ & $c_{u_{4 R}}=-0,135$ \\
$d_{4 R}$ & $c_{d_{4 R}}=-0,105$ \\
$t_{L} e b_{L}$ & $c_{q_{3}}=0,07$ \\
$t_{R}$ & $c_{t_{R}}=-0,2$ \\
$b_{R}$ & $c_{b_{R}}=-0,501$ \\
$c_{L} e s_{L}$ & $c_{q_{2}}=0,501$ \\
$c_{R}$ & $c_{c_{R}}=-0,501$ \\
$s_{R}$ & $c_{s_{R}}=-0,525$ \\
$u_{L} e d_{L}$ & $c_{q_{1}}=0,51$ \\
$u_{R}$ & $c_{u_{R}}=-0,5865$ \\
$d_{R}$ & $c_{d_{R}}=-0,545$ \\
\hline
\end{tabular}

A matriz CKM estendida é, nesse caso (desconsiderando-se as fases decorrentes da diagonalização das matrizes de massa):

$$
\mathbf{V}=\left(\begin{array}{cccc}
0,97419 & 0,2257 & 0,00357 & 0,00126984 \\
0,226 & 0,973 & 0,0393 & 0,00103526 \\
0,0051 & 0,039 & 0,984 & 0,174425 \\
0,00193009 & 0,00554734 & 0,174334 & 0,984669
\end{array}\right)
$$

Para o segundo caso: 


Tabela 3 - Caso 2: $m_{u_{4}}=600 \mathrm{Ge}$
\begin{tabular}{|c|c|}
\hline Campo & Constante $\mathrm{c}$ \\
\hline$u_{4 L} e d_{4 L}$ & $c_{q_{4}}=0,02$ \\
$u_{4 R}$ & $c_{u_{4 R}}=-0,0245$ \\
$d_{4 R}$ & $c_{d_{4 R}}=-0,04$ \\
$t_{L} e b_{L}$ & $c_{q_{3}}=0,09$ \\
$t_{R}$ & $c_{t_{R}}=-0,22$ \\
$b_{R}$ & $c_{b_{R}}=-0,501$ \\
$c_{L} e s_{L}$ & $c_{q_{2}}=0,501$ \\
$c_{R}$ & $c_{c_{R}}=-0,501$ \\
$s_{R}$ & $c_{s_{R}}=-0,525$ \\
$u_{L} e d_{L}$ & $c_{q_{1}}=0,51$ \\
$u_{R}$ & $c_{u_{R}}=-0,5865$ \\
$d_{R}$ & $c_{d_{R}}=-0,545$ \\
\hline
\end{tabular}

A matriz CKM estendida é, nesse caso:

$$
\mathbf{V}=\left(\begin{array}{cccc}
0,97424 & 0,2255 & 0,00382 & 0,00122927 \\
0,225 & 0,97 & 0,0410 & 0,00105882 \\
0,0055 & 0,041 & 0,998 & 0,0566325 \\
0,00127034 & 0,00100608 & 0,0566326 & 0,998394
\end{array}\right)
$$

Em ambos os casos, as matrizes CKM estendidas são compatíveis com a matriz CKM determinada experimentalmente, equação (4.50), como era de se esperar pela própria construção.

\subsection{Acoplamentos}

Com os parâmetros c's dos quarks determinados, bem como suas matrizes de massa e a matriz CKM estendida, podem ser verificadas as consequências fenomenológicas do modelo. Retomando alguns dos resultados mais relevantes, segue que a expansão da interação entre o campo do glúon e os quarks tem a forma:

$$
\begin{aligned}
S_{f b} & =-g_{00} \int d^{4} x \bar{\psi}^{(0)}(x) \gamma^{\mu} \mathbf{T}^{a} \psi^{(0)} A_{\mu}^{a(0)}(x)- \\
& -g_{01} \int d^{4} x \bar{\psi}^{(0)}(x) \gamma^{\mu} \mathbf{T}^{a} \psi^{(0)} A_{\mu}^{a(1)}(x)+\ldots
\end{aligned}
$$


e os novos acoplamentos introduzidos pelo modelo podem ser colocados na forma (retomando a equação (4.15)):

$$
g_{00}=g
$$

sendo este o acoplamento do glúon (modo zero) da QCD e

$$
\begin{array}{rl}
g_{01}=g \int_{0}^{\pi k R} d & y \frac{\left(1 \mp 2 c_{L, R}\right)}{\left(e^{\left(1 \mp 2 c_{L, R}\right) \pi k R}-1\right)} \frac{e^{2\left(1 \mp c_{L, R}\right) y}}{N_{1}} . \\
\cdot\left[\mathrm{J}_{1}\left(\frac{m_{n}}{k} e^{y}\right)+b_{1} \mathrm{Y}_{1}\left(\frac{m_{n}}{k} e^{y}\right)\right],
\end{array}
$$

com:

$$
N_{n}^{2}=\frac{1}{\pi R} \int_{0}^{\pi R} d y e^{2 k y}\left[\mathrm{~J}_{1}\left(\frac{m_{n}}{k} e^{k y}\right)+b_{1} \mathrm{Y}_{1}\left(\frac{m_{n}}{k} e^{k y}\right)\right]^{2}
$$

e

$$
b_{1}=-\frac{\mathrm{J}_{1}\left(\frac{m_{n}}{k}\right)+\frac{m_{n}}{k} \mathrm{~J}_{1}^{\prime}\left(\frac{m_{n}}{k}\right)}{\mathrm{Y}_{1}\left(\frac{m_{n}}{k}\right)+\frac{m_{n}}{k} \mathrm{Y}_{1}^{\prime}\left(\frac{m_{n}}{k}\right)}
$$

com:

$$
b_{1}\left(m_{n}\right)=b_{1}\left(m_{n} e^{\pi k R}\right)
$$

relembrando que dada a forma da interação, o campo vetorial deve ser par sob as transformações $y \rightarrow-y$.

O acoplamento $g_{01}$, dos modos de Kaluza-Klein do glúon com os modos zero dos quarks, é o principal a ser analisado neste projeto, uma vez que é o mais forte e que interações desse tipo trocam sabor por correntes neutras (dado também que a escala de energias é $\sim 1 \mathrm{TeV}$ ). Isto torna importantes, para verificação experimental, os processos que envolvem esse acoplamento, porque devem ser os primeiros a serem observados no LHC e porque permitem descartar toda uma classe de modelos, caso esteja em desacordo com os experimentos. Assim sendo, o trabalho em curso caminha para a determinação de seções de choque de alguns processos, visando testar essa teoria.

Se a condensação de quarks por meio de uma interação forte é o mecanismo de quebra da simetria eletrofraca e de geração das massas dos férmions, o sinal mais distintivo dessa teoria é a troca de sabor a nível árvore pelas interações com o primeiro modo KK do glúon [9].

Pela equação (4.54), percebe-se que os acoplamentos entre os quarks e o glúon (modo zero do campo) são os mesmos para todos os quarks. Entretanto 
a equação (4.55) mostra que, como cada campo tem uma constante $c_{L, R} \mathrm{e}$ mais ainda, como cada constante depende da quiralidade, a interação entre o modo KK do glúon e cada quark é quiral e não universal, ou seja, depende do sabor.

Desta maneira, não é possível fazer a composição como na equação (4.33), sabendo que na equação (4.53) cada um dos campos $u^{\prime}, c^{\prime}, t^{\prime}, u_{4}^{\prime}$ possui um acoplamento $g_{10}$ correspondente, diferente, em geral, dos demais, implicando que cada campo desses respeita individualmente $S U(3)_{c}$ mas não o vetor $U^{\prime}$, após a integração da dimensão extra.

Este impedimento em se compor o vetor $U^{\prime}$ implica, por sua vez, que não pode haver o cancelamento, nas interações do segundo termo do lado direito da equação (4.53), dado pela unitariedade das matrizes $\mathbf{S}$, que aparecem, por exemplo, na equação (4.35), cancelamento que, como foi visto, é responsável pela ausência de correntes neutras que trocam sabor no Modelo Padrão.

Deve-se decompor o vetor $U^{\prime}$ e cada componente seu deve-se escrever como combinação linear dos outros campos do mesmo tipo (tipo $u$ ou tipo $d$ ) e de mesma quiralidade. Procedendo dessa forma, segue que, para o quark up de mão esquerda vale:

$$
u_{L}^{\prime}=\left(\mathbf{S}_{L}^{u}\right)_{11} u_{L}+\left(\mathbf{S}_{L}^{u}\right)_{12} c_{L}+\left(\mathbf{S}_{L}^{u}\right)_{13} t_{L}+\left(\mathbf{S}_{L}^{u}\right)_{14} u_{4 L},
$$

e de maneira análoga ocorre para todos os outros campos de quark, sabendo que as matrizes $\mathbf{S}$ foram determinadas para a caracterização dos próprios quarks (conforme a seção anterior, onde as matrizes $\mathbf{S}$ são combinadas para compor a matriz CKM estendida).

Assim os acoplamentos entre quarks e o primeiro modo KK do glúon, para os quarks agora na base de auto-estados de massa, serão obtidos a partir da lagrangeana:

$$
-\mathcal{L}=g_{01 u_{L}} \bar{u}_{L} \gamma_{\mu} \mathbf{T}^{a}\left[\left(\mathbf{S}_{L}^{u}\right)_{11} u_{L}+\left(\mathbf{S}_{L}^{u}\right)_{12} c_{L}+\left(\mathbf{S}_{L}^{u}\right)_{13} t_{L}+\left(\mathbf{S}_{L}^{u}\right)_{14} u_{4 L}\right] A^{a \mu}+\ldots
$$

Nessa equação foi mostrado apenas o primeiro acoplamento, para o quark up. Os termos omitidos são os acoplamentos faltantes, ou seja, os acoplamentos para os demais quarks do tipo up $\left(c, t, u_{4}\right)$ e para todos os quarks do tipo $d\left(d, s, b, d_{4}\right)$. Os acoplamentos, efetivamente, vão depender não apenas do valor obtido da equação (4.15), mas também dos componentes das matrizes S. 
Deve ser observada uma aproximação na equação (4.60), usual na literatura, de que apenas o campo à direita da matriz $\gamma_{\mu}$ foi expandido em termos dos outros quarks na base de massa, correspondendo à aproximação de que, os coeficientes da expansão, são pequenos e quando multiplicados entre si resultam em termos desprezíveis em comparação ao termo dominante. Falta então, para completar o modelo, a determinação das constantes de acoplamento $g_{01}$. As constantes foram introduzidas na equação 4.15;

$$
\begin{array}{rl}
g_{01}=g \int_{0}^{\pi k R} d & y \frac{\left(1 \mp 2 c_{L, R}\right)}{\left(e^{\left(1 \mp 2 c_{L, R}\right) \pi k R}-1\right)} \frac{e^{2\left(1 \mp c_{L, R}\right) y}}{N_{1}} . \\
\cdot\left[\mathrm{J}_{1}\left(\frac{m_{n}}{k} e^{y}\right)+b_{1} \mathrm{Y}_{1}\left(\frac{m_{n}}{k} e^{y}\right)\right] .
\end{array}
$$

Seja a equação acima e $g_{01 u_{4 L}}=g g_{u_{4 L}}$, com $g$ o acoplamento do glúon (e de maneira análoga para todos os outros quarks). Esta definição, aplicada às constantes da tabela 2 , resulta (para o caso 1 ) em:

Tabela 4 - Caso 1: $m_{u_{4}}=500 \mathrm{GeV}$

\begin{tabular}{|c|c|c|}
\hline$g_{u_{4 L}}=g_{d_{4 L}}=4,11166$ & $g_{u_{4 R}}=3,4859$ & $g_{d_{4 R}}=3,66193$ \\
\hline$g_{t_{L}}=g_{b_{L}}=3,85543$ & $g_{t_{R}}=3,06862$ & $g_{b_{R}}=0,380096$ \\
\hline$g_{c_{L}}=g_{s_{L}}=0,380096$ & $g_{c_{R}}=0,380096$ & $g_{s_{R}}=0,272814$ \\
\hline$g_{u_{L}}=g_{d_{L}}=0,330327$ & $g_{u_{R}}=0,214052$ & $g_{d_{R}}=0,233931$ \\
\hline
\end{tabular}

e para o caso 2, aplicando a equação (4.15) à tabela 3:

Tabela 5 - Caso 2: $m_{u_{4}}=600 \mathrm{GeV}$
\begin{tabular}{|c|c|c|}
\hline$g_{u_{4 L}}=g_{d_{4 L}}=4,11166$ & $g_{u_{4 R}}=4,08951$ & $g_{d_{4 R}}=4,01187$ \\
\hline$g_{t_{L}}=g_{b_{L}}=3,74636$ & $g_{t_{R}}=2,92933$ & $g_{b_{R}}=0,380096$ \\
\hline$g_{c_{L}}=g_{s_{L}}=0,380096$ & $g_{c_{R}}=0,380096$ & $g_{s_{R}}=0,272814$ \\
\hline$g_{u_{L}}=g_{d_{L}}=0,330327$ & $g_{u_{R}}=0,214052$ & $g_{d_{R}}=0,233931$ \\
\hline
\end{tabular}

Pode ser notado também que os acoplamentos aumentam, em geral, conforme aumenta a massa da partícula, reflexo da localização da função de onda do férmion na quinta dimensão. Deve ser enfatizado que para se obter essas constantes de acoplamento é necessário determinar as constantes características de todos os quarks a partir da correta determinação de suas matrizes de massa e da matriz CKM. Para possibilitar a verificação da teoria, faz-se necessário um estudo fenomenológico dos resultado obtidos até o presente. 


\section{Capítulo 5}

\section{Fenomenologia}

O Modelo Padrão segue como limite, em baixas energias, da teoria desenvolvida até aqui, em particular no capítulo anterior. O passo seguinte é submeter a teoria a testes experimentais. O que se busca é a observação de sinais experimentais desta teoria que tem o setor eletrofraco fortemente acoplado e do mecanismo de geração de massas.

Para viabilizar os testes da teoria e dos modelos aqui apresentados, bem como para orientar as procuras de sinais experimentais, é necessário um estudo fenomenológico, cujos resultados servirão para cumprir os dois objetivos.

Devido à impossibilidade de observação do primeiro modo KK do glúon como uma ressonância [9], é necessário utilizar o fato de este modo trocar sabor a nível árvore por correntes neutras (FCNC). Neste capítulo são estudados dois processos onde ocorre troca de sabor e que podem ser postos à prova no LHC, a saber: $p p \rightarrow u_{4} \bar{t}$ e $p p \rightarrow d_{4} \bar{b}$.

Por simulação pelo método de Monte Carlo são obtidas seções de choque para os dois processos e, com o decaimento dos quarks a estados finais convenientes, são estudadas distribuições de grandezas relacionadas aos decaimentos, tais quais momento transverso ou massas invariantes. O fundo é comparado com o sinal para essas distribuições, levando ao desenvolvimento de estratégias de cortes para a diminuição do fundo e, por fim, mostrando a viabilidade de observação do sinal no LHC. Os principais resultados apresentados no final do capítulo anterior e principalmente neste, são divulgados na referência [37]. 


\section{$5.1 \quad$ Processos}

Para as simulações, serão adotados valores para a massa do primeiro modo de Kaluza-Klein do glúon, $m_{1}$, que até o presente ficou indeterminada. Sabese, por um lado, que $m_{1}$ deve ser maior do que $2 \mathrm{TeV}$, para evitar problemas com parâmetros de precisão eletrofracos ( $\mathrm{S}$ e T) [33]. Por outro lado, $m_{1}$ não deve ser muito superior a $3 \mathrm{TeV}$, pois seria recuperado o problema da hierarquia, como pode ser observado pela relação entre $m_{1} \mathrm{e} k$, na equação (3.138). Serão estudados inicialmente dois casos: $m_{1}=2 \mathrm{TeV}$ e $m_{1}=3 \mathrm{TeV}$, por serem representativos dos limites de massa esperados para o primeiro modo de Kaluza-Klein do glúon.

Os processos de interesse para testes do modelo empregado são aqueles onde se pode observar o modo KK do glúon, pois uma suposta quarta geração pode estar presente, independente de qual seja o modelo que substitui o Modelo Padrão, mas modos de Kaluza-Klein, se observados, constituem evidência em favor do modelo aqui proposto.

Como foi observado, os maiores acoplamentos do primeiro modo KK do glúon $\left(G_{1}\right)$ são aqueles com os quarks $u_{4}$ e $d_{4}$. A seção de choque fica, então, favorecida para processos que envolvam o $u_{4}$, o $d_{4}$ e o $G_{1}$. Os processos mais óbvios, dados os acoplamentos e os elementos da CKM estendida, seriam $p p \rightarrow u_{4} \bar{u}_{4}$ e $p p \rightarrow d_{4} \bar{d}_{4}$, mostrando o $G_{1}$ como uma ressonância. Entretanto, no LHC, o fundo irredutível da QCD domina os processos (ref. [9]) e, portanto, a descoberta da quarta geração de quarks deve ocorrer antes da detecção de $G_{1}$.

A largura do modo KK do glúon é alta, devido aos altos acoplamentos, o que desfigura a distribuição de Breit-Wigner de seu espalhamento. O fundo é muito expressivo no LHC e, dadas as incertezas nas distribuições, o sinal, que apareceria como excesso à distribuição do fundo, não pode ser diferenciado de flutuações estatísticas da distribuição do fundo, a menos que se obtenha suficiente luminosidade acumulada no LHC. Mostra-se, na referência [9], que a luminosidade necessária para a observação é proibitiva, não sendo viável identificar o sinal por este método.

Outro método utiliza o fato de o $G_{1}$ violar a conservação de sabor dos quarks. Isto é uma característica destes modelos (derivados de RandallSundrum) e a primeira (e possivelmente a única) maneira de se observar o 
primeiro modo excitado do glúon (o que significa uma interação forte que condensa um ou dois quarks da quarta geração). Nesta direção foram estudados dois processos, a saber: $p p \rightarrow u_{4} \bar{t}$ e $p p \rightarrow d_{4} \bar{b}$.

Como este modelo é a única realização conhecida de teorias onde ocorre a condensação de uma quarta geração de quarks por meio de interação forte, a importância da detecção de processos que trocam sabor se torna clara, pois esta é, possivelmente, a única maneira de verificação experimental da classe de teorias à qual pertence o modelo, no LHC.

\subsubsection{Processo $p p \rightarrow u_{4} \bar{t}$}

Um dos processos mais interessantes, pois deve ser um dos primeiros a serem observados no LHC é:

$$
p p \rightarrow u_{4} \bar{t}
$$

que inclui, como um de seus sub-processos principais:

$$
q \bar{q} \rightarrow u_{4} \bar{t}
$$

com o diagrama de Feynman mostrado na figura 5.1. Nota-se, em particular, a troca de sabor por meio do $G_{1}$ (o primeiro modo de Kaluza-Klein do glúon), que é neutro.

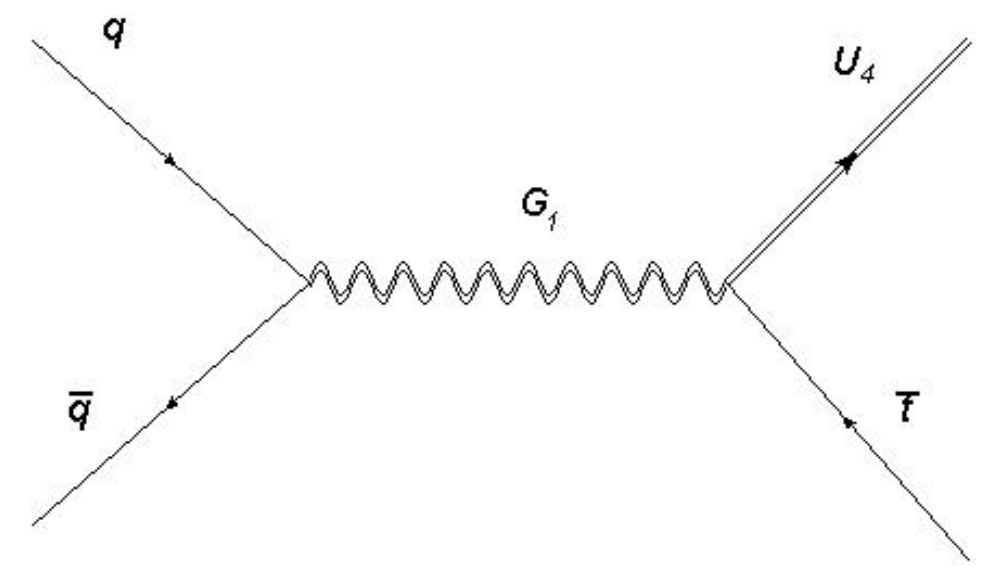

Figura 5.1: Diagrama de Feynman para o processo $q \bar{q} \rightarrow u_{4} \bar{t}$.

Esse processo de troca de sabor domina sobre os outros pois o acoplamento de $u_{4} \operatorname{com} G_{1}$ e a troca de sabor de $u_{4}$ por $t$ dada pelo elemento da 
matriz de rotação $\mathbf{S}$, são altos, o que eleva a seção de choque de sua produção. Portanto, deve ser um dos primeiros a ser observados.

Inicialmente são calculadas as seções de choque, variando os parâmetros que foram determinados para o modelo. Para tanto foi utilizado um sistema de simulação computacional, baseado no método de Monte Carlo [10]. O software que implementa tal simulação é o MadEvent [38], que, utilizando-se de integração por Monte Carlo, fornece arquivos com os 4-momentos de cada partícula envolvida no estado final. Além disso, fornece a seção de choque total com a respectiva incerteza, consequência da incerteza estatística no método de Monte Carlo.

Todos os eventos foram gerados aplicando-se o corte de seleção de $p_{T}(b)>30 \mathrm{GeV}$. Evidentemente processos que não possuam $b$ 's antes de completado o decaimento não são afetados por este corte. Foram usados outros cortes padronizados na versão 4.2.11 do MadEvent. Esta versão utiliza, como padrão, a PDF de código cteq611. Foram utilizadas as escalas de renormalização e de fatorização fixas e iguais a 91,1880 GeV (massa do bóson Z), também escalas padrão na versão referida do software.

As seções de choque são:

Tabela 6 - Seções de choque (em fb) $p p \rightarrow u_{4} \bar{t} ; E_{C M}=14 \mathrm{TeV}$

\begin{tabular}{|c|c|c|}
\hline massas & $m_{u_{4}}=500 \mathrm{GeV}$ & $m_{u_{4}}=600 \mathrm{GeV}$ \\
\hline$m_{1}=2 \mathrm{TeV}$ & 232 & 91 \\
\hline$m_{1}=3 \mathrm{TeV}$ & 44 & 18 \\
\hline
\end{tabular}

para a energia do centro de massa $(\mathrm{CM}) E=14 \mathrm{TeV}$, máximo esperado para o LHC, utilizando-se os cortes básicos do MadEvent.

Para a energia do centro de massa $E_{C M}=7 \mathrm{TeV}$ :

Tabela 7 - Seções de choque (em fb) $p p \rightarrow u_{4} \bar{t} ; E_{C M}=7 \mathrm{TeV}$

\begin{tabular}{|c|c|c|}
\hline massas & $m_{u_{4}}=500 \mathrm{GeV}$ & $m_{u_{4}}=600 \mathrm{GeV}$ \\
\hline$m_{1}=2 \mathrm{TeV}$ & 29,5 & 10,6 \\
\hline$m_{1}=3 \mathrm{TeV}$ & 4,8 & 1,7 \\
\hline
\end{tabular}




\subsubsection{Processo $p p \rightarrow d_{4} \bar{b}$}

O segundo processo estudado:

$$
p p \rightarrow d_{4} \bar{b}
$$

mostrou-se ainda mais interessante do que o primeiro, por causa de sua topologia, como será visto na próxima seção.

O diagrama de Feynman de um dos principais sub-processos é mostrado na figura 5.2. Nota-se, novamente, a troca de sabor por meio do $G_{1}$.

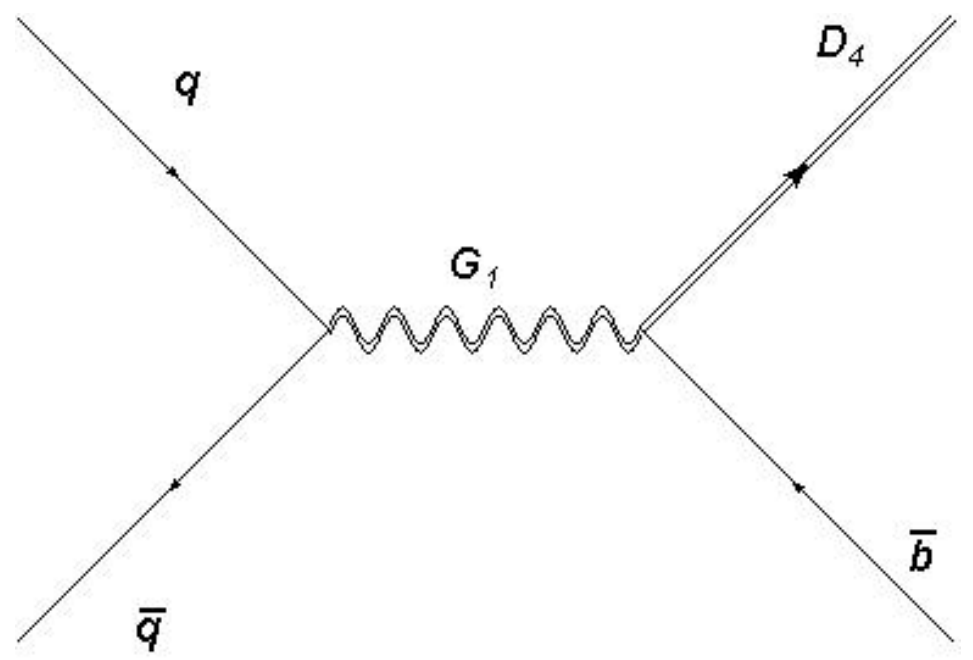

Figura 5.2: Diagrama de Feynman para o processo $q \bar{q} \rightarrow d_{4} \bar{b}$.

Simulações com o MadEvent levam às seções de choque para a energia do centro de massa $(\mathrm{CM}) E=14 \mathrm{TeV}$.

Tabela 8 - Seções de choque (em fb) $p p \rightarrow d_{4} \bar{b} ; E_{C M}=14 \mathrm{TeV}$

\begin{tabular}{|c|c|c|}
\hline massas & $m_{d_{4}}=450 \mathrm{GeV}$ & $m_{d_{4}}=550 \mathrm{GeV}$ \\
\hline$m_{1}=2 \mathrm{TeV}$ & 78,6 & 47,0 \\
\hline$m_{1}=3 \mathrm{TeV}$ & 15,2 & 9,1 \\
\hline
\end{tabular}


Para a energia do centro de massa $E_{C M}=7 \mathrm{TeV}$ :

Tabela 9 - Seções de choque (em fb) $p p \rightarrow d_{4} \bar{b} ; E_{C M}=7 \mathrm{TeV}$

\begin{tabular}{|c|c|c|}
\hline massas & $m_{d_{4}}=450 \mathrm{GeV}$ & $m_{d_{4}}=550 \mathrm{GeV}$ \\
\hline$m_{1}=2 \mathrm{TeV}$ & 10,47 & 5,64 \\
\hline$m_{1}=3 \mathrm{TeV}$ & 1,73 & 0,91 \\
\hline
\end{tabular}

A análise e os cortes adicionais, para ambos os processos, serão estudados para o caso onde $m_{u 4}=500 \mathrm{GeV}, m_{1}=2 \mathrm{TeV}$ e para a energia, no centro de massa, de $14 \mathrm{TeV}$. A análise pode ser prontamente realizada para os demais casos mostrados nesta tese e ainda mais, pode ser realizada para casos de valores intermediários. Contudo, por definição e para demonstrar a viabilidade de detecção deste tipo de processo no LHC e, portanto, da observação da interação forte que gera a condensação dos quarks, o estudo foi especializado para um caso, neste trabalho.

\subsection{Detecção de $p p \rightarrow d_{4} \bar{b}$}

A topologia deste processo é distinta daquela para o processo $p p \rightarrow u_{4} \bar{t}$, havendo, para o processo que envolve $d_{4}$, um jato de $b$ que provém diretamente de $G_{1}$, enquanto nenhum dos jatos de $b$ para o processo que envolve $u_{4}$ possui tal procedência. O processo $p p \rightarrow d_{4} \bar{b}$ possui ainda um diferencial para a detecção de $G_{1}$ : o jato de $b$ que se origina diretamente de $G_{1}$ é muito energético (leva grande parte da energia de $G_{1}$ ), comparado com as demais partículas e jatos, sendo esta a principal característica distintiva deste processo.

\subsubsection{Decaimento}

Primeiramente, considera-se o decaimento $d_{4} \rightarrow t W^{-}$, por ser o único apreciável, visto que o vértice envolve a quarta coluna da matriz CKM aumentada, da qual apenas os elementos $V_{44}$ e $V_{34}$ são relevantes, sendo apenas o último aquele que representa a mistura que envolve o $W^{-}$.

Foi exigido um lépton carregado do decaimento do $W$, para passar por um dos triggers do LHC. Do $W^{-}$resultante de $d_{4}$, foi exigido o decaimento 
em léptons, particularmente em elétrons, pois o elétron resultante seria mais energético do que o pósitron do decaimento subsequente de $W^{+}$proveniente de $\bar{t}$. A consideração do múon dobra os valores aqui apresentados (para o sinal e para o fundo) e processos envolvendo o tau não foram considerados por resultarem em decaimentos que tornam complicada sua identificação.

Por fim, o único decaimento relevante para o quark top é o decaimento em quark bottom, por razões análogas às do decaimento de $d_{4}$, requerendo-se que o $W^{+}$resultante decaia em jatos.

Então, dentre os possíveis produtos de decaimento do processo, foi estudado o decaimento dado pelo diagrama de Feynman da figura 5.3.

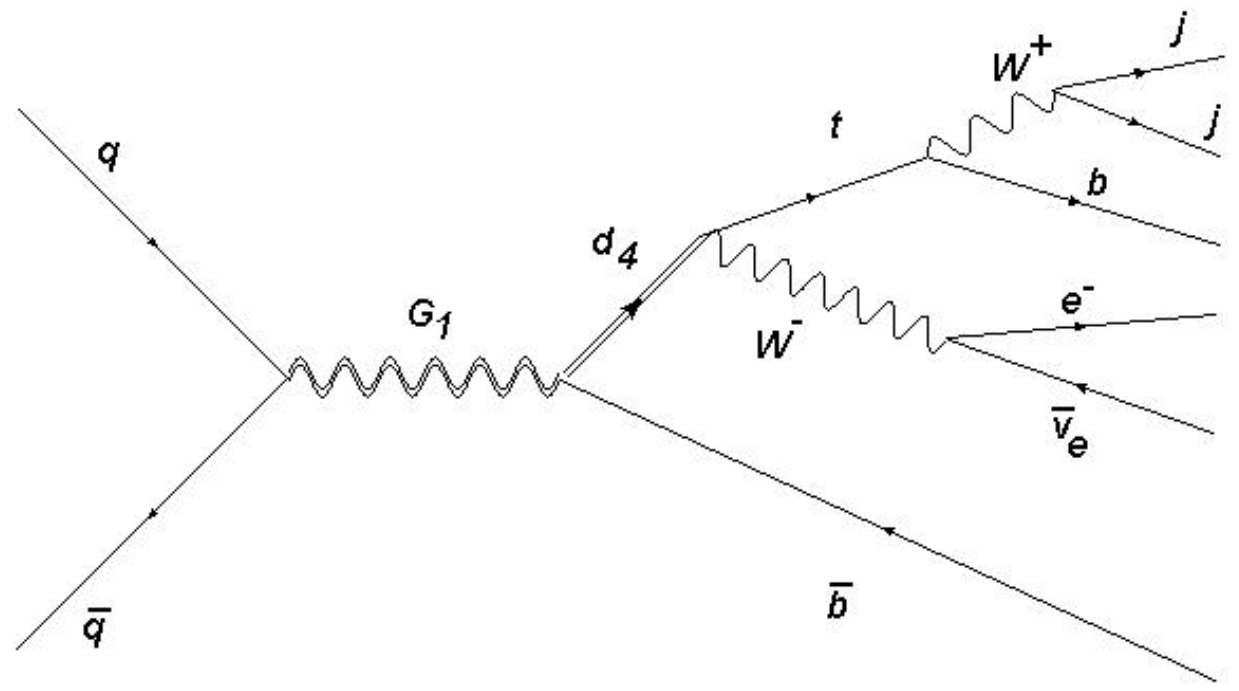

Figura 5.3: Diagrama de Feynman de canal de decaimento para detecção de $q \bar{q} \rightarrow d_{4} \bar{b}$.

Apesar de não possuir fundo irredutível, o processo apresenta fundo redutível muito grande, sendo necessária sua identificação e a elaboração de estratégias de corte para sua redução. O principal fundo identificado para este sinal é dado pelo processo no diagrama de Feynman da figura 5.4.

Como mencionado anteriormente, para o estudo dos decaimentos e desenvolvimento da estratégia de cortes, a discussão foi especializada para um dos casos introduzidos neste capítulo e no anterior, o caso onde $m_{1}=2 \mathrm{TeV}$ e $m_{u 4}=500 \mathrm{GeV}$, além de ser utilizada a energia de $14 \mathrm{TeV}$ no centro de 


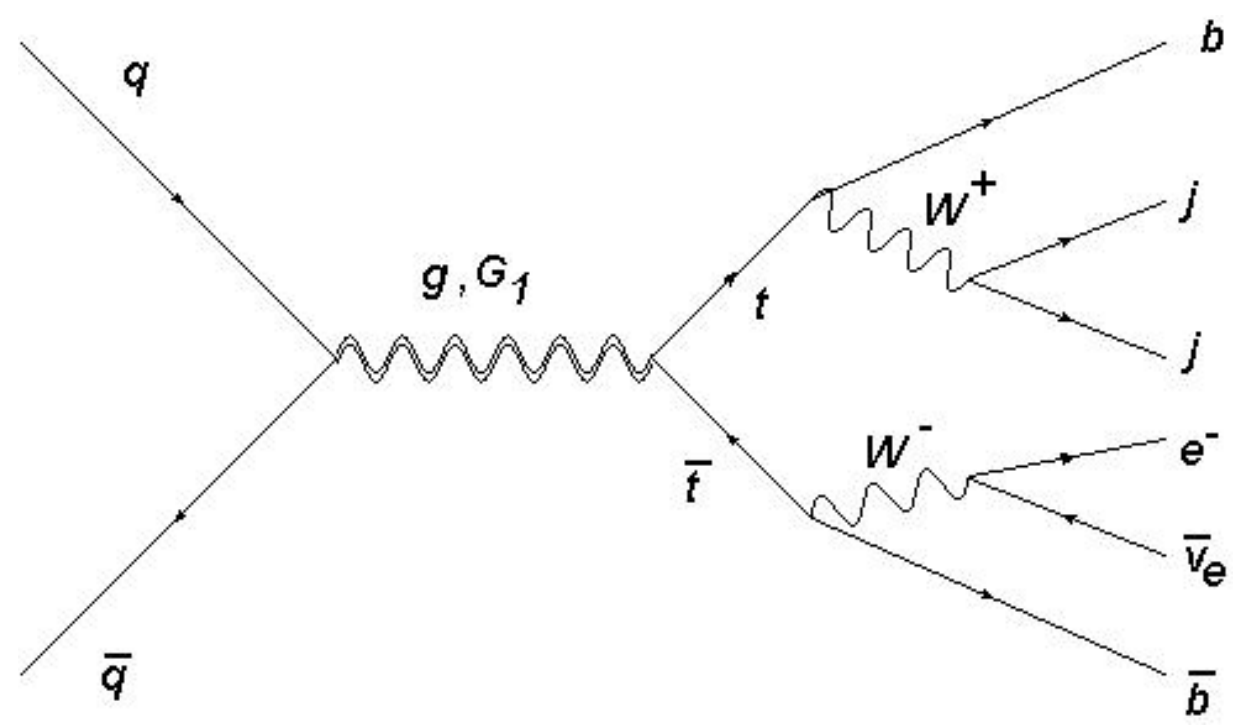

Figura 5.4: Diagrama de Feynman de canal de decaimento para $t \bar{t}$, como fundo para $d_{4} \bar{b}$.

massa. Uma vez que o LHC foi projetado para trabalhar por tempo limitado a $7 \mathrm{TeV}$, a luminosidade acumulada deve ser menor do que para $14 \mathrm{TeV}$, o que torna a observação do processo mais favorável nesta última situação. Para a geração dos eventos foi utilizado o programa MadEvent [38], cujos eventos foram utilizados nos programas Bridge [39] e Decay para gerar os decaimentos.

As seções de choque para o sinal e para o fundo decaídos aos estados finais mostrados nas figuras 5.3 e 5.4 são dadas na tabela 10, para $14 \mathrm{TeV}$ no centro de massa. Foram gerados $2 \times 10^{5}$ eventos para o sinal e $2 \times 10^{6}$ eventos para o fundo.

Tabela 10 - Seções de choque (em fb) para sinal e fundo de $p p \rightarrow d_{4} \bar{b}$

\begin{tabular}{|c|c|}
\hline \multicolumn{2}{|c|}{$E_{C M}=14 \mathrm{TeV}$} \\
\hline sinal & fundo \\
\hline$\sigma_{S}=5,4 \mathrm{fb}$ & $\sigma_{B}=5,4.10^{4} \mathrm{fb}$ \\
\hline
\end{tabular}




\subsubsection{Análise e cortes}

A análise do decaimento foi realizada para $14 \mathrm{TeV}$ de energia no centro de massa. Foram consideradas as seguintes variáveis:

- $p_{T}(i)$ : momento transverso da partícula $i$ (componente do momento no plano transverso à direção do feixe).

- $H_{T}$ : definida como a soma escalar do momento transverso de todas as partículas mais $E_{T}$ ausente (missing $E_{T}$ ).

- $m(i, j, k, \ldots)$ : massa invariante das partículas $i, j, k, \ldots$, definida por:

$$
m(i, j, k, \ldots)=\sqrt{p_{i, j, k, \ldots}^{2}}
$$

onde

$$
p_{i, j, k, \ldots}=p_{i}+p_{j}+p_{k}+\ldots
$$

e $p_{i}$ é o 4-momento da partícula $i$.

- $\Delta R_{i j}$ : distância das partículas $i, j$ no plano $\Delta \varphi_{i j} \Delta \eta_{i j}$ :

$$
\Delta R_{i j}=\sqrt{\Delta \varphi_{i j}^{2}+\left(\eta_{i}-\eta_{j}\right)^{2}},
$$

onde $\Delta \varphi_{i j}$ é o ângulo entre as partículas no plano transversal ao feixe e $\eta_{i}$ é a pseudo-rapidez da partícula $i$, dada por:

$$
\eta_{i}=-\ln \left[\tan \left(\theta_{i} / 2\right)\right]
$$

sendo $\theta_{i}$ o ângulo entre a trajetória da partícula $i$ e a direção do feixe.

Além destas variáveis, algumas outras (rapidez, ângulo) são mostradas como padrão pelo MadAnalysis e foram estudadas, a princípio, para comparar sinal e fundo e auxiliar o desenvolvimento da estratégia de cortes, para observação do sinal. Entretanto, as variáveis listadas acima se mostraram mais interessantes e, na verdade, foram as únicas utilizadas para os cortes.

Para este processo, as figuras 5.5, 5.6, 5.7, 5.8 e 5.9 mostram as distribuições mais relevantes para o presente estudo, antes de qualquer corte. Nesta nomenclatura, $b 1$ significa o jato de bottom mais energético, $b 2$ seria 
o segundo jato de bottom mais energético e assim sucessivamente, inclusive para as demais partículas. São mostradas, simultaneamente, as distribuições para o sinal (preto) e fundo (vermelho).

Para encontrar a estratégia de cortes é necessário eliminar o máximo possível do fundo pela imposição de limites para as grandezas determinadas, enquanto se mantém o máximo possível de sinal. Isto equivale a um problema de otimização, entretanto resolvido de maneira artesanal, sem o formalismo associado.

Como nos detectores não há maneira clara de vincular experimentalmente cada jato a um determinado processo, $b 1$ pode representar ora o bottom que vem diretamente de $G_{1}$, ora o bottom que vem de $t$. Assim sendo, ocorre a dispersão dos valores pela distribuição.

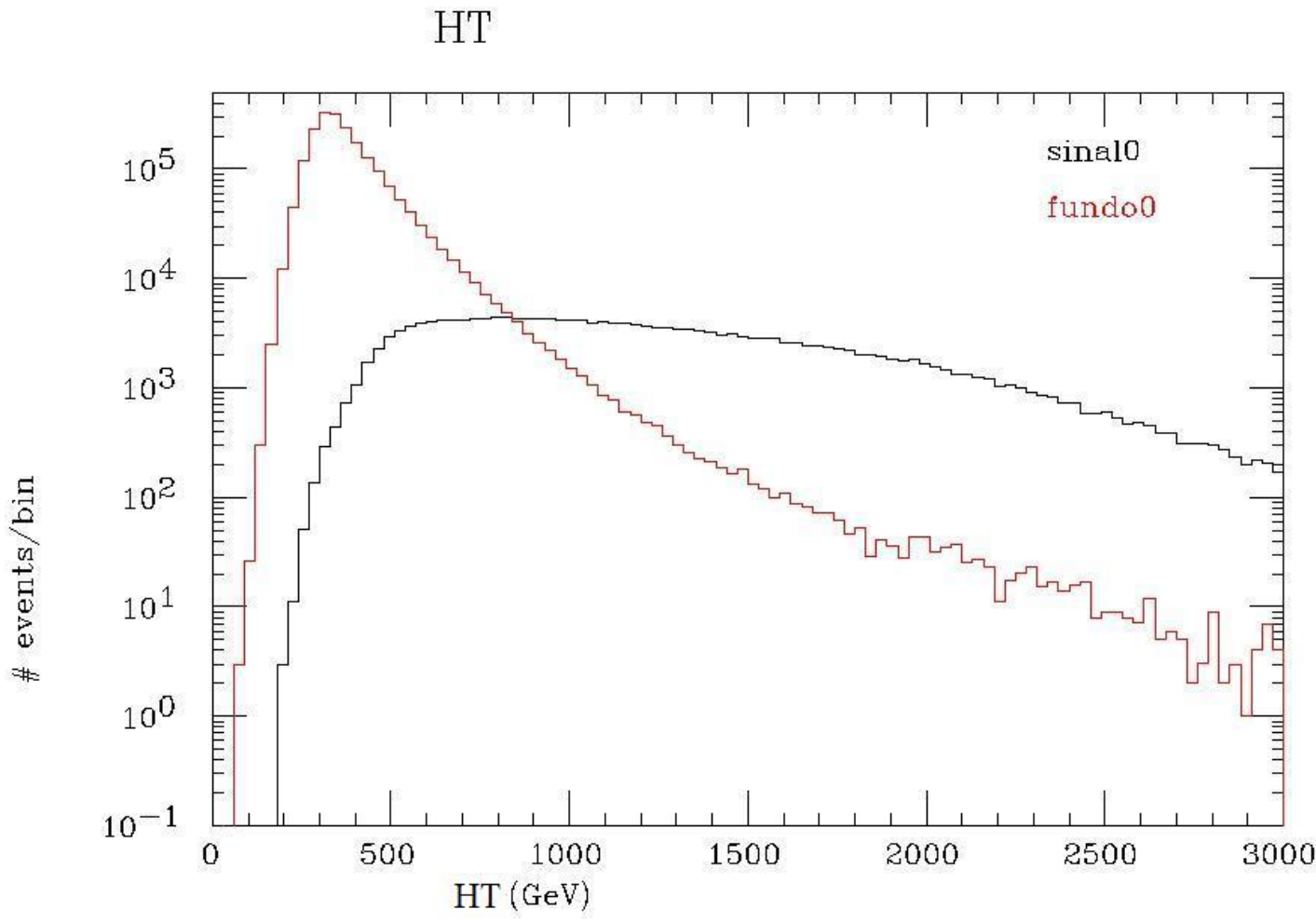

Figura 5.5: $H_{T}$ antes dos cortes.

O gráfico da figura 5.5 mostra distinção clara entre sinal e fundo, este último possui um pico em $H_{T}$ para valores menores, característica não apre- 
sentada pelo sinal.

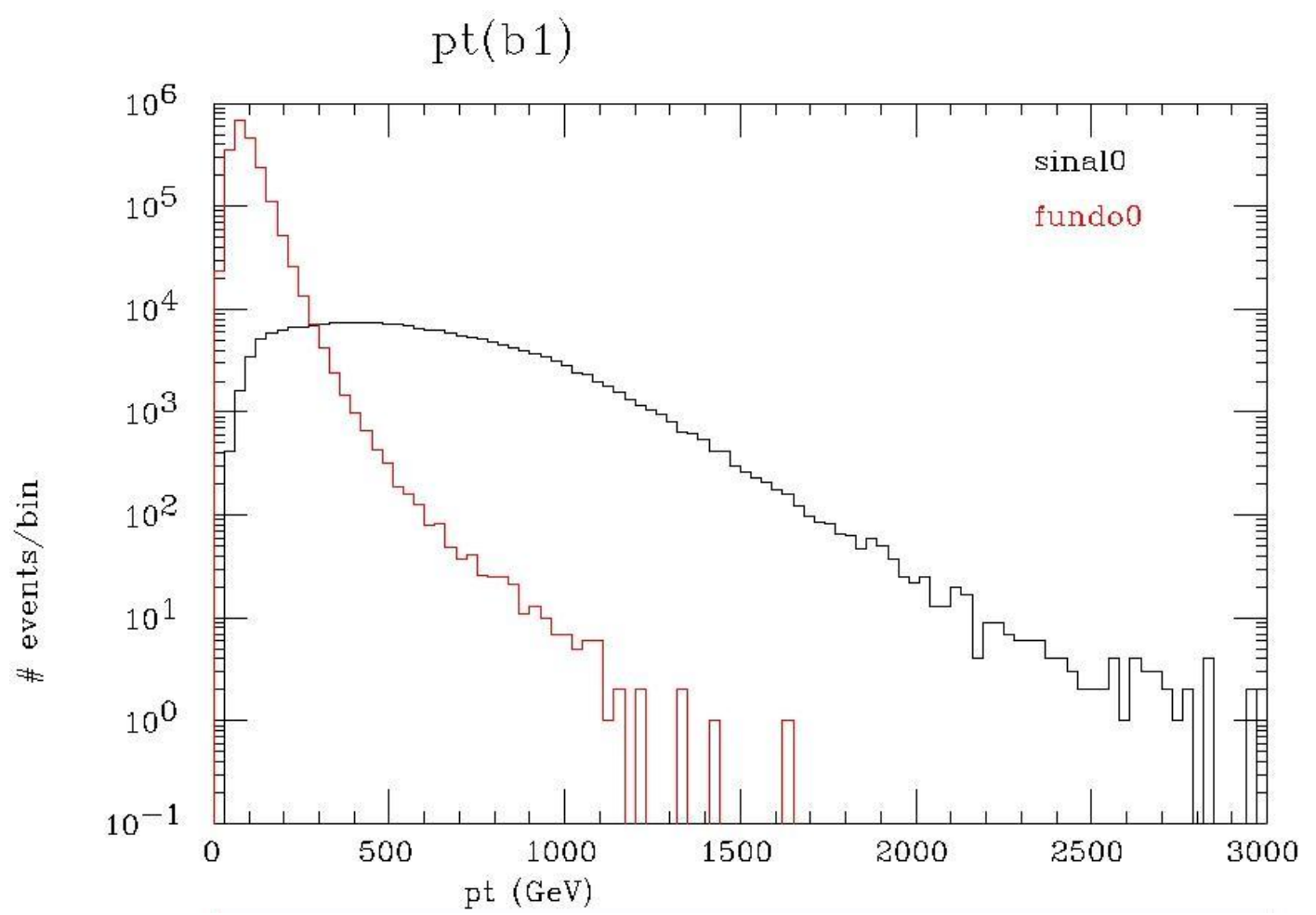

Figura 5.6: $p_{T}(b 1)$ antes dos cortes.

Na figura 5.6 nota-se que o pico do fundo, para valores menores de $p_{T}$, é mais pronunciado para o $p_{T}(b 1)$ do que para $H_{T}$, sendo então um melhor candidato ao primeiro corte, por serem eliminados mais eventos do fundo com respeito ao sinal. 


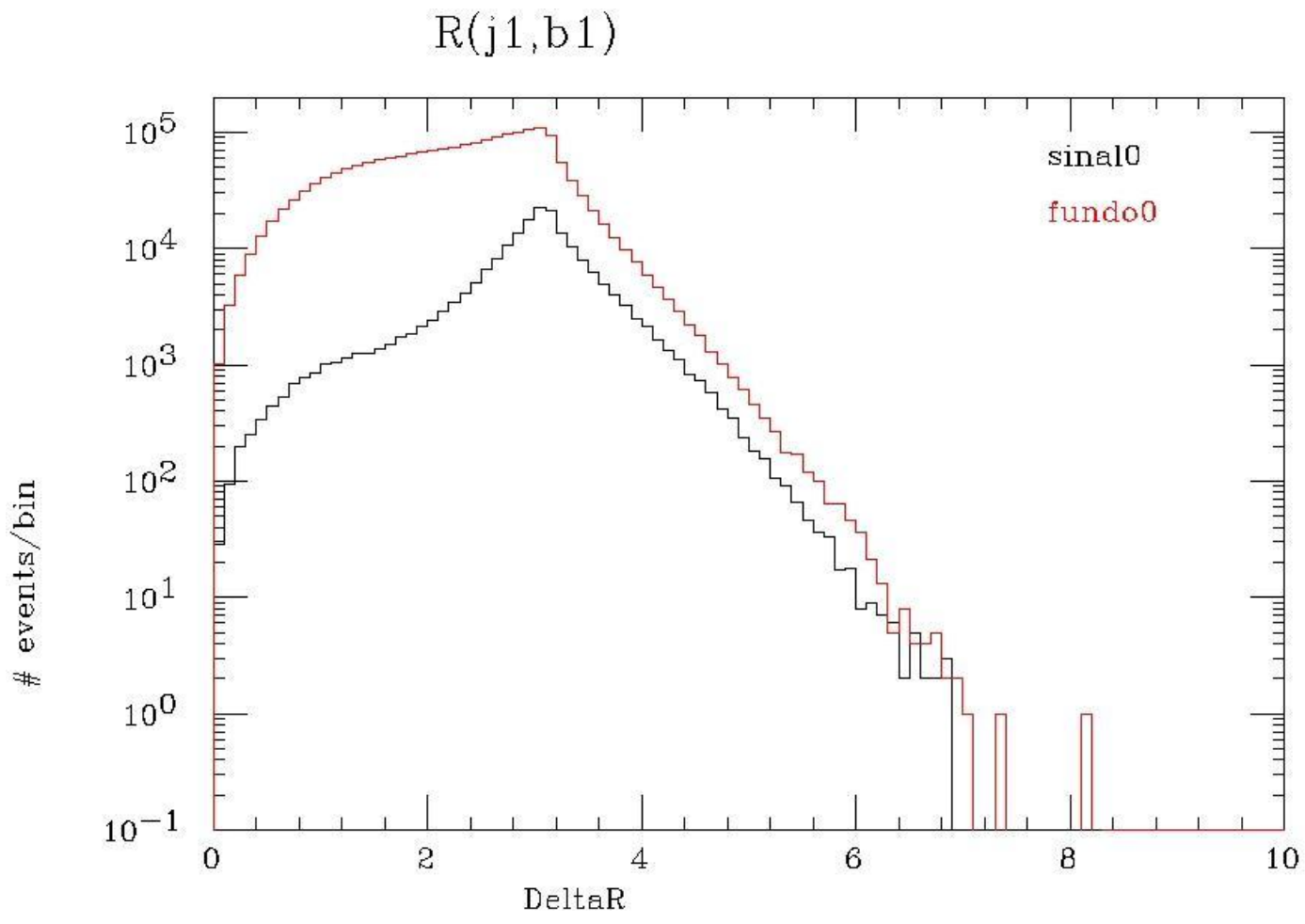

Figura 5.7: $\Delta R_{j 1 b 1}$ antes dos cortes.

A figura 5.7 mostra um excesso para o fundo, com respeito ao sinal, para pequenos valores de Delta $R$, mas os picos das duas distribuições estão no mesmo valor da abscissa e as diferenças não são muito distintivas, de maneira que esta distribuição não é uma candidata muito interessante a um primeiro corte. 


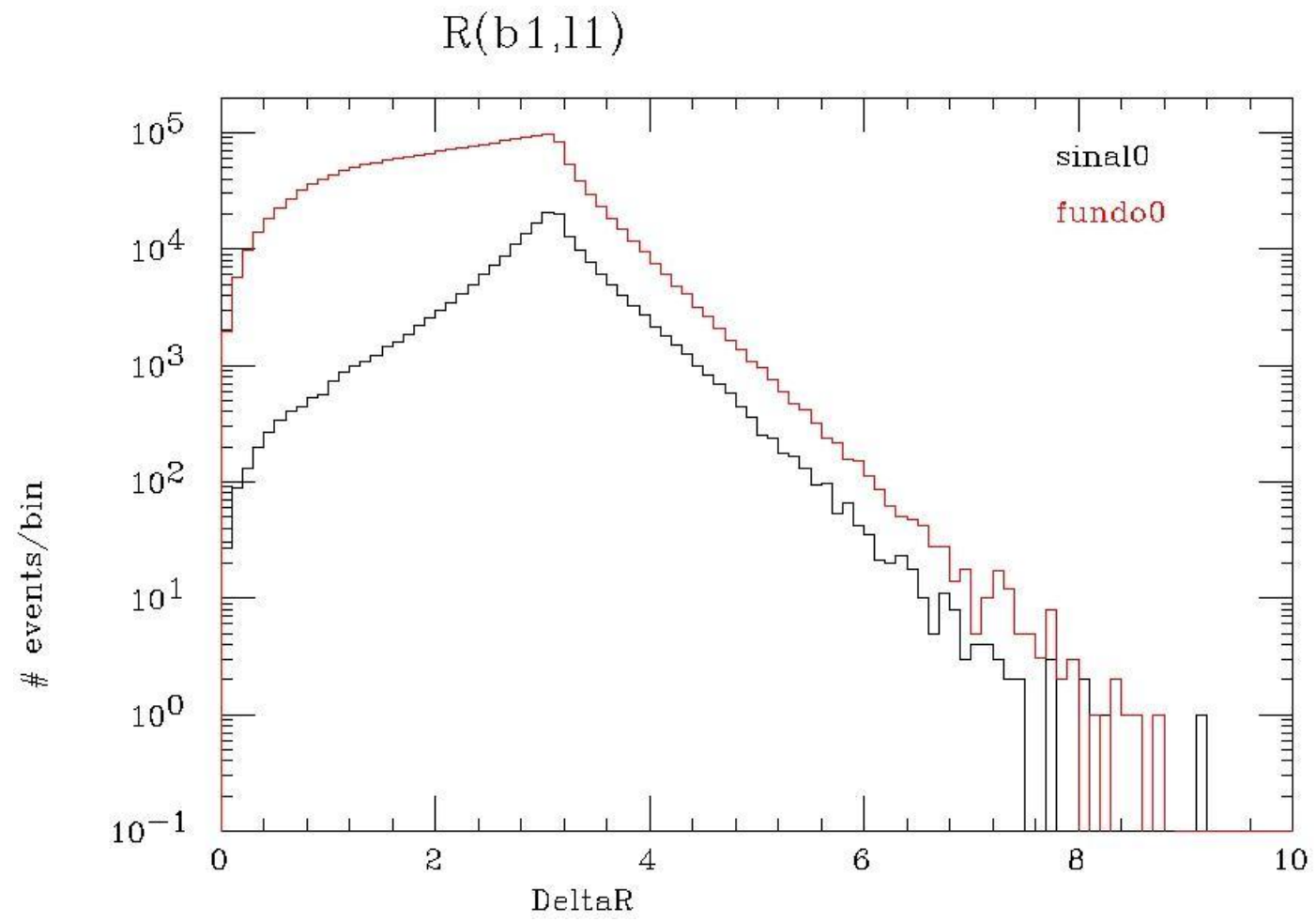

Figura 5.8: $\Delta R_{b 1 l 1}$ antes dos cortes.

Novamente a distribuição de Delta R, agora entre $b(1)$ e o elétron, não mostra grandes distinções entre sinal e fundo, de forma que esta distribuição também não oferece boa possibilidade de um primeiro corte. 


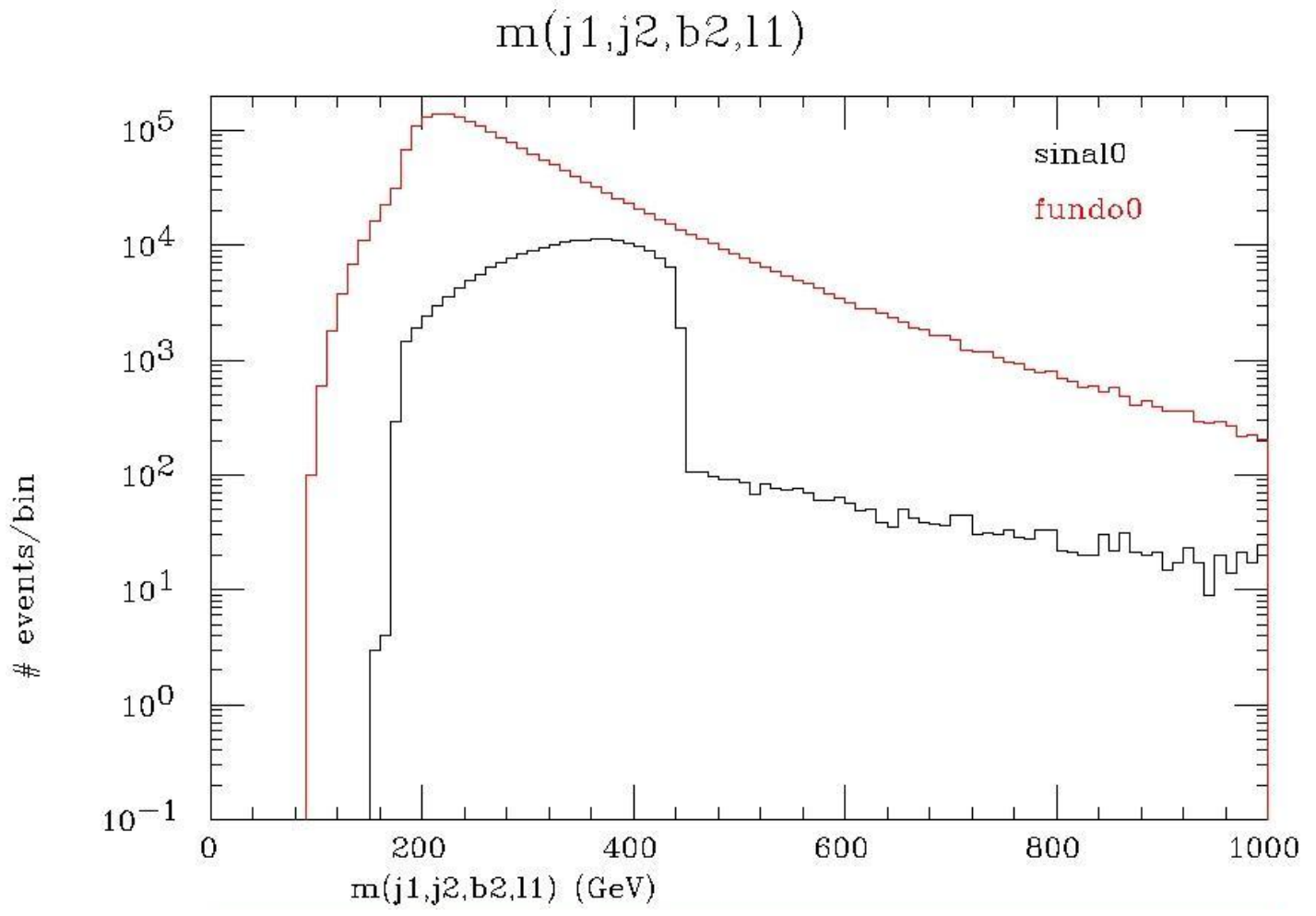

Figura 5.9: $m(j 1, j 2, b 2, l 1)$ antes dos cortes.

A distribuição da massa invariante entre os dois jatos, o bottom menos energético e o elétron tem como característica, para o sinal, queda brusca no número de eventos (denominada limite ou endpoint) para $m=450 \mathrm{GeV}$, denunciando a origem destas partículas como sendo o $D_{4}$. Este tipo de distribuição ocorre por que o decaimento tem ainda um neutrino, que não é detectado. O fundo não possui tal característica, mas por outro lado é muito superior ao sinal. Esta distribuição é, mesmo assim, muito distintiva do sinal com relação ao fundo.

Inicialmente poder-se-ia, pela análise das figuras, aplicar o corte em $H_{T}$. Entretanto, utilizando-se o fato de o $b_{1}$ ser muito energético, o primeiro corte foi aplicado em $p_{T}(b 1)$. Desta maneira, foram considerados, após o primeiro corte, apenas eventos com $p_{T}(b 1)>500 \mathrm{GeV}$. 
As figuras 5.10, 5.11, 5.12, 5.13 e 5.14 mostram as mesmas variáveis após o primeiro corte.

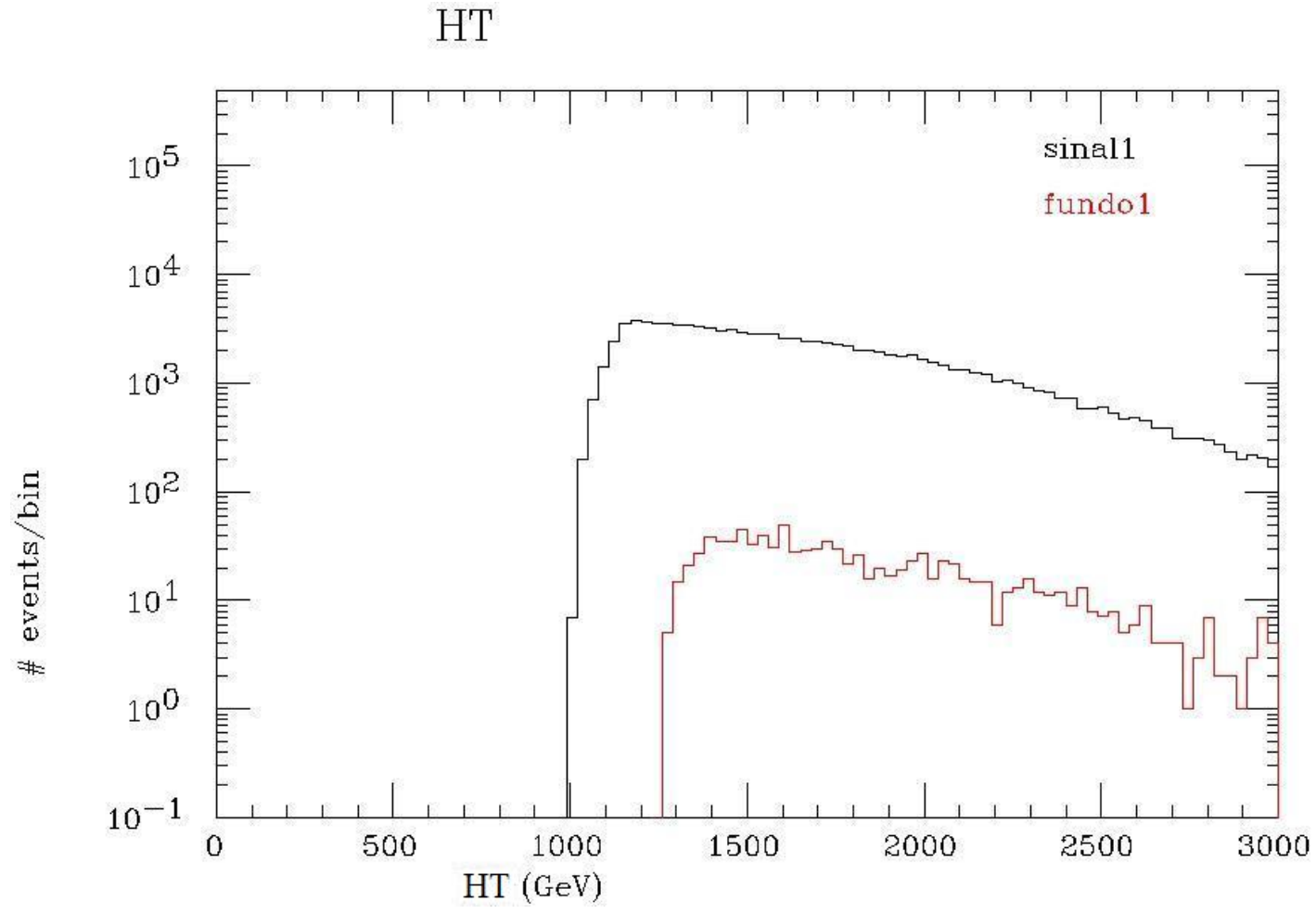

Figura 5.10: $H_{T}, p_{T}(b 1)>500 \mathrm{GeV}$.

Observa-se, após o primeiro corte, a drástica redução do fundo, que passou a contar com cerca de mil eventos (1025 eventos restantes) em sua distribuição, que inicialmente possuía $2 \times 10^{6}$ eventos, enquanto o sinal perdeu menos da metade de seus 200 mil eventos iniciais (restaram 108327 eventos). A forma das distribuições não sugere um corte em $H_{T}$. 


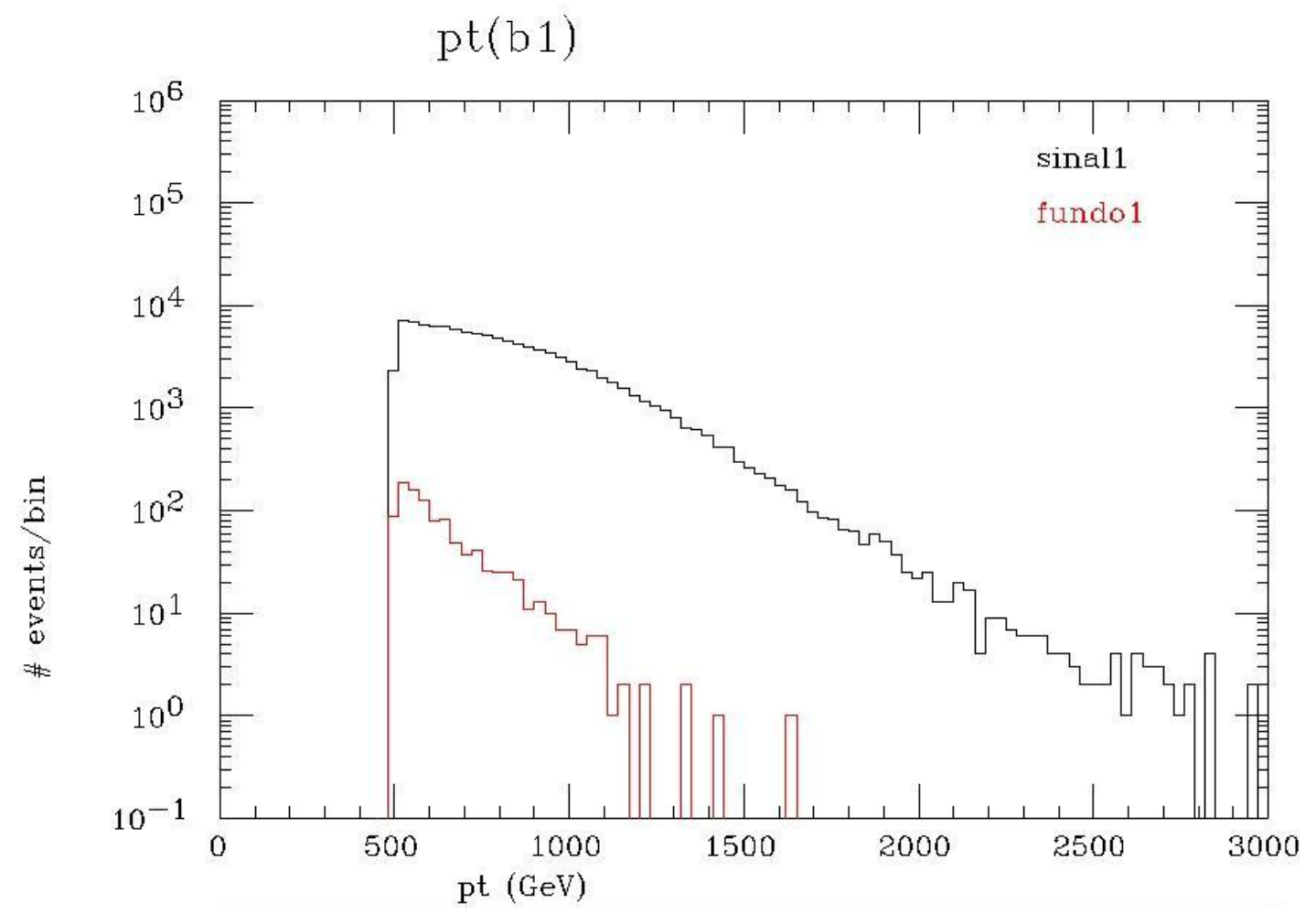

Figura 5.11: $p_{T}(b 1), p_{T}(b 1)>500 \mathrm{GeV}$.

A distribuição tem a mudança óbvia que o corte introduziu, ou seja, apenas eventos nos quais o bottom mais energético possuir momento transverso maior do que $500 \mathrm{GeV}$ são considerados. As distribuições não sugerem novos cortes. 


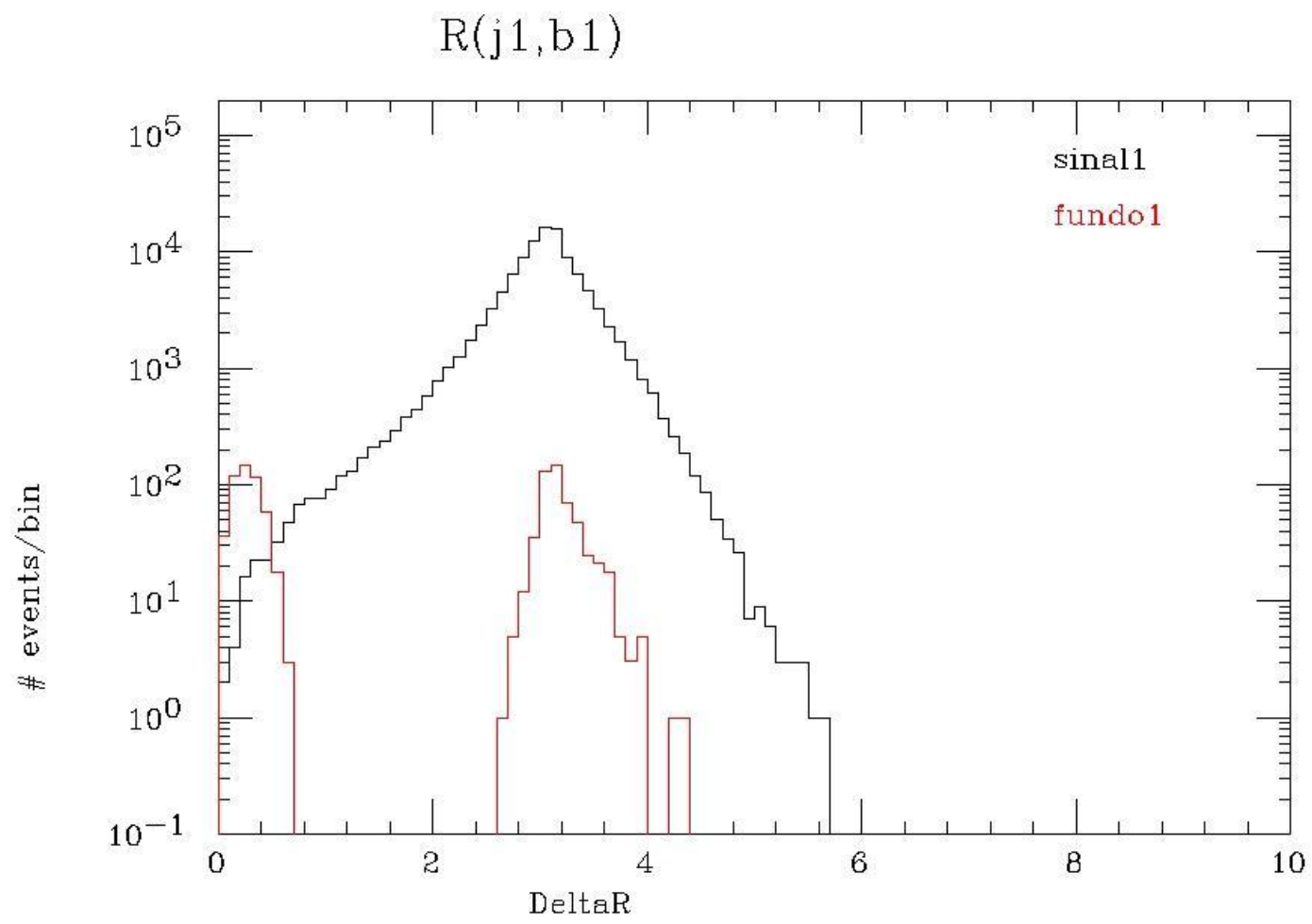

Figura 5.12: $\Delta R_{j 1 b 1}, p_{T}(b 1)>500 \mathrm{GeV}$.

Após o primeiro corte, a distribuição de Delta R (j1,b1) apresenta característica muito distintiva entre sinal e fundo: o fundo possui dois picos, sendo um deles para pequenos valores de Delta $R$, o que sugere um novo corte, pelo qual se elimina grande quantidade de fundo e pequena de sinal. Há, para o fundo, a presença de dois picos, caso um dos quarks $b$ do decaimento (figura 5.4) seja muito energético $\left(p_{T}>500 \mathrm{GeV}\right)$. Neste caso há, então, duas situações distintas predominantes: em uma delas, o jato mais energético (não proveniente de b) está em direção muito próxima à do jato mais energético de $b$ (ambos provêm predominantemente do mesmo top) ou, na segunda situação possível, a direção do jato dista de um ângulo de aproximadamente $\Theta \approx \Delta R \approx \pi$ (cada um provém de um top distinto). Este é o único caso predominante para o sinal (caso em que o jato mais energético de $b$ se origina de $G_{1}$ e o jato mais energético (não b-jet) tem origem no decaimento de t, predominantemente). 


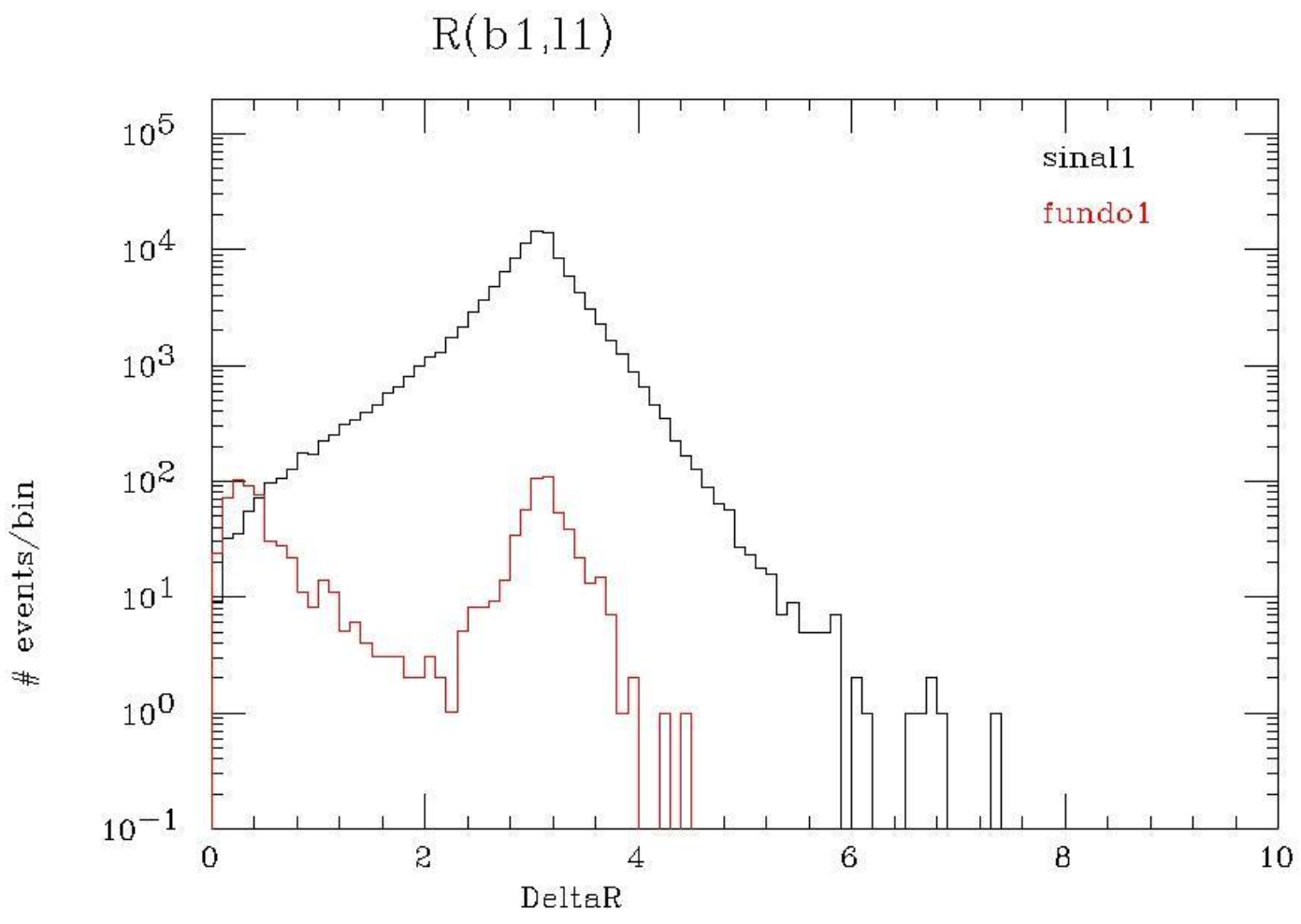

Figura 5.13: $\Delta R_{b 1 l 1}, p_{T}(b 1)>500 \mathrm{GeV}$.

Nesta distribuição de Delta $R(b 1,11)$ há a presença de dois picos na distribuição do fundo, com apenas um pico para o sinal, o que também a torna discriminante. Entretanto, a distribuição anterior se mostra mais favorável a aplicação de cortes. Como no caso anterior, a razão de dois picos para o fundo está na origem do b-jet mais energético e do lépton mais energético; caso se originem do mesmo top, Delta $\mathrm{R}$ deve ser menor (próximo a zero), caso se originem um de $t$ o outro de $\bar{t}$, deve predominar $\Delta R \approx \pi$, único caso que predomina para o sinal. 


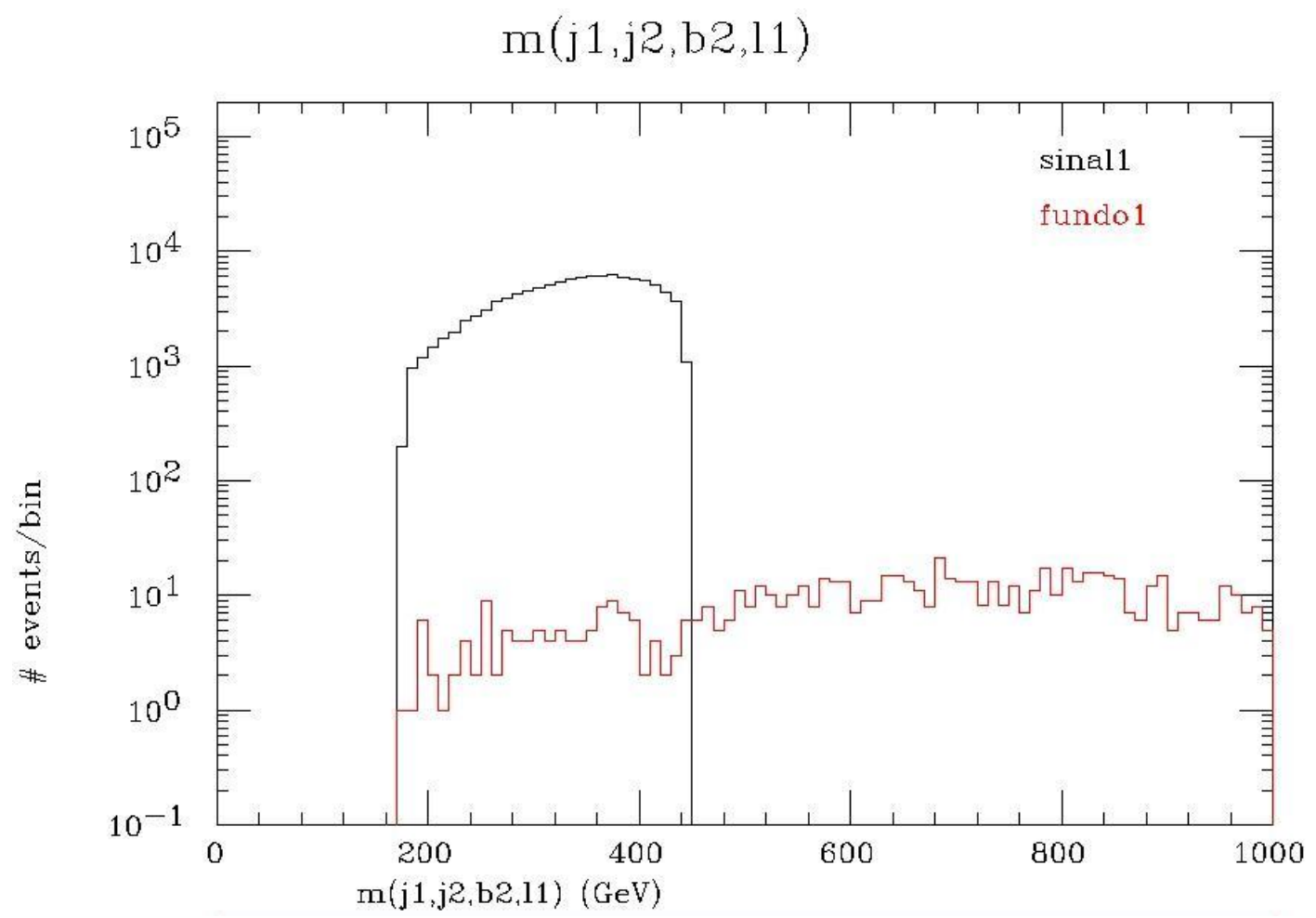

Figura 5.14: $m(j 1, j 2, b 2, l 1), p_{T}(b 1)>500 \mathrm{GeV}$.

Nota-se, na distribuição de massa invariante das quatro partículas, que o sinal mostra claramente (devido ao limite da massa invariante) a origem comum das quatro como sendo uma partícula de massa $450 \mathrm{GeV}\left(d_{4}\right)$, enquanto o fundo não apresenta nada semelhante.

Deve ser enfatizado que após o primeiro corte, dos $2 \times 10^{6}$ eventos presentes inicialmente no fundo, restaram apenas $\sim 10^{3}$ (1025) eventos, uma redução muito significativa (cerca de 2000 vezes). O sinal teve uma redução de cerca de metade dos seus eventos presentes inicialmente, de $2 \times 10^{5}$ para cerca de $\sim 10^{5}\left(1,08327 \times 10^{5}\right)$ eventos.

São notáveis ainda outras características: as 2 distribuições de $\Delta R$ (após o primeiro corte), mostrados nos gráficos 5.12 e 5.13, apresentam, para o fundo, dois picos distintos, o que sugere novos cortes. Também pode ser notado que a forma da distribuição da massa invariante para as quatro partículas (gráfico 5.14) tem, no caso do sinal, um limite em $450 \mathrm{GeV}$, o que é sinal característico do decaimento em 5 partículas a partir de uma partícula de massa $450 \mathrm{GeV}$ 
$\left(d_{4}\right)$, servindo como clara distinção entre sinal e fundo e mostrando a origem das 5 partículas (com o neutrino não detectado) sendo o $d_{4}$.

O próximo corte é feito em $\Delta R_{j 1 b 1}$. A presença de dois picos no gráfico 5.12 , em particular do pico para valores menores de $\Delta R$, sugere que seja feito um corte na região, de forma a eliminar mais fundo do que sinal. Então, aplicando o corte $\Delta R_{j 1 b 1}>0,7$ resultam os gráficos $5.15,5.16,5.17,5.18$ e 5.19 .

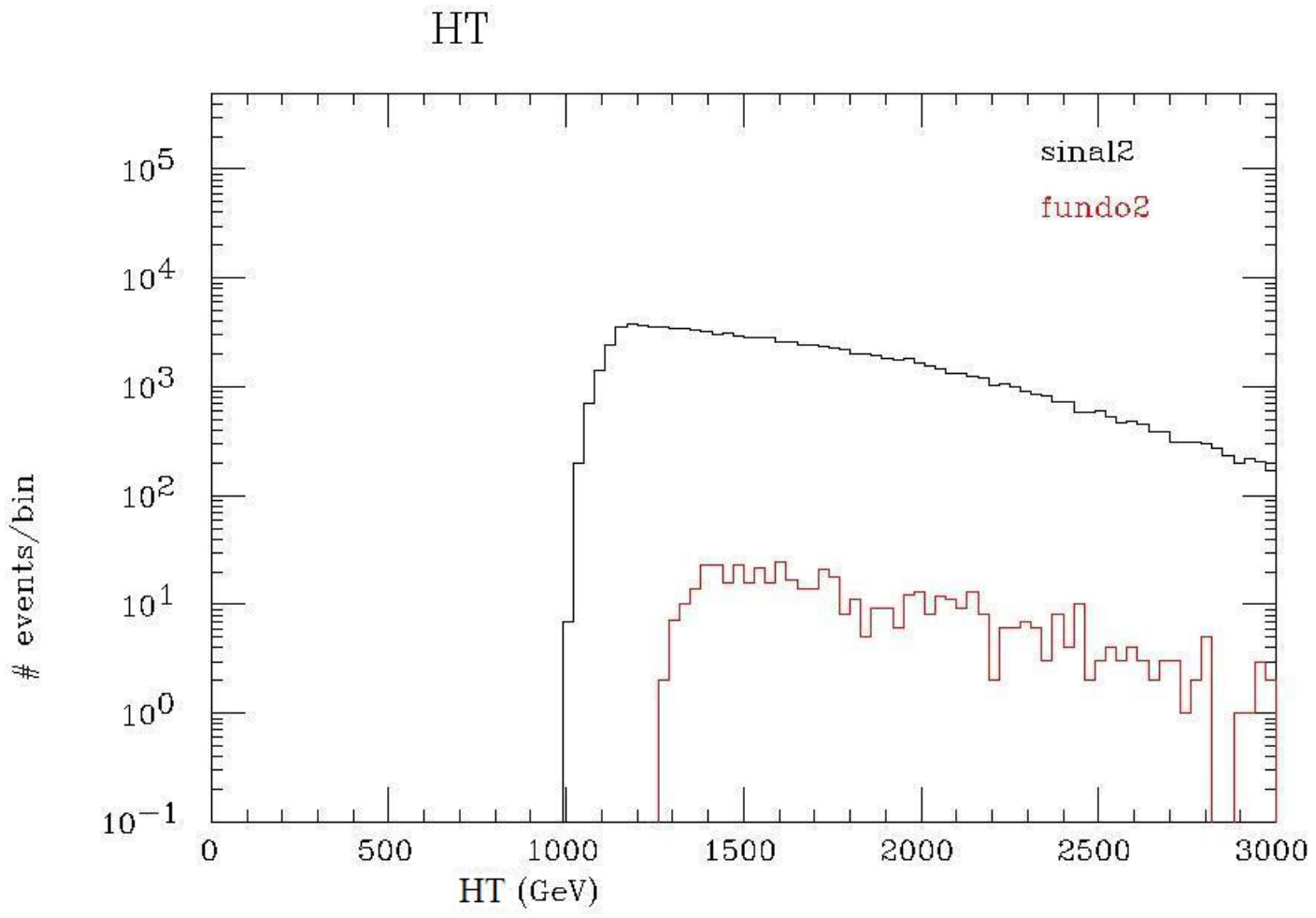

Figura 5.15: $H_{T}, p_{T}(b 1)>500 \mathrm{GeV}, \Delta R_{j 1 b 1}>0,7$.

Após o segundo corte, a única característica notável das novas distribuições de $H_{T}$ é a redução do fundo e praticamente a manutenção do sinal. 


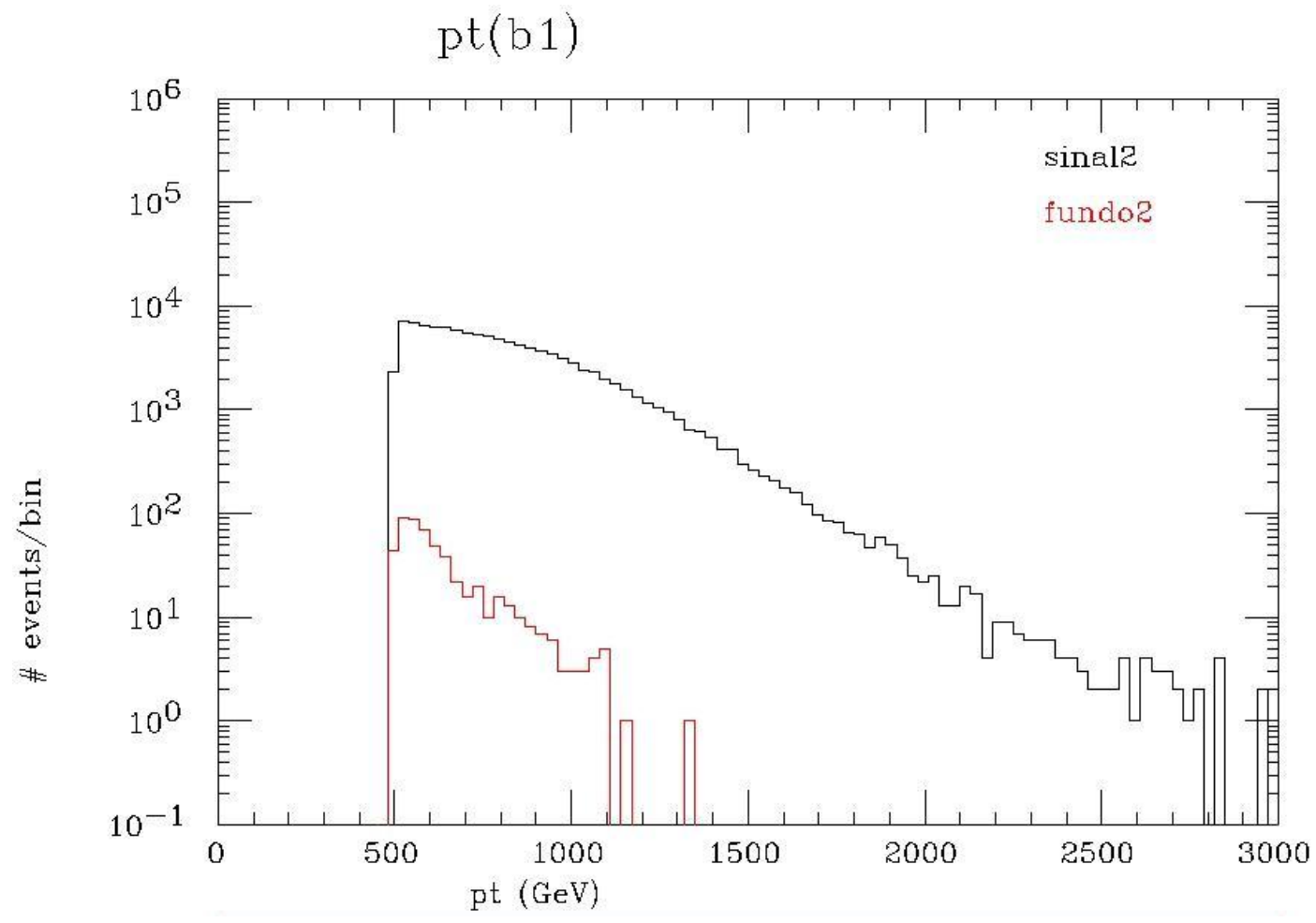

Figura 5.16: $p_{T}(b 1), p_{T}(b 1)>500 \mathrm{GeV}, \Delta R_{j 1 b 1}>0,7$.

Como no caso anterior, nota-se apenas a redução do fundo no novo gráfico de $p_{T}(b 1)$. 


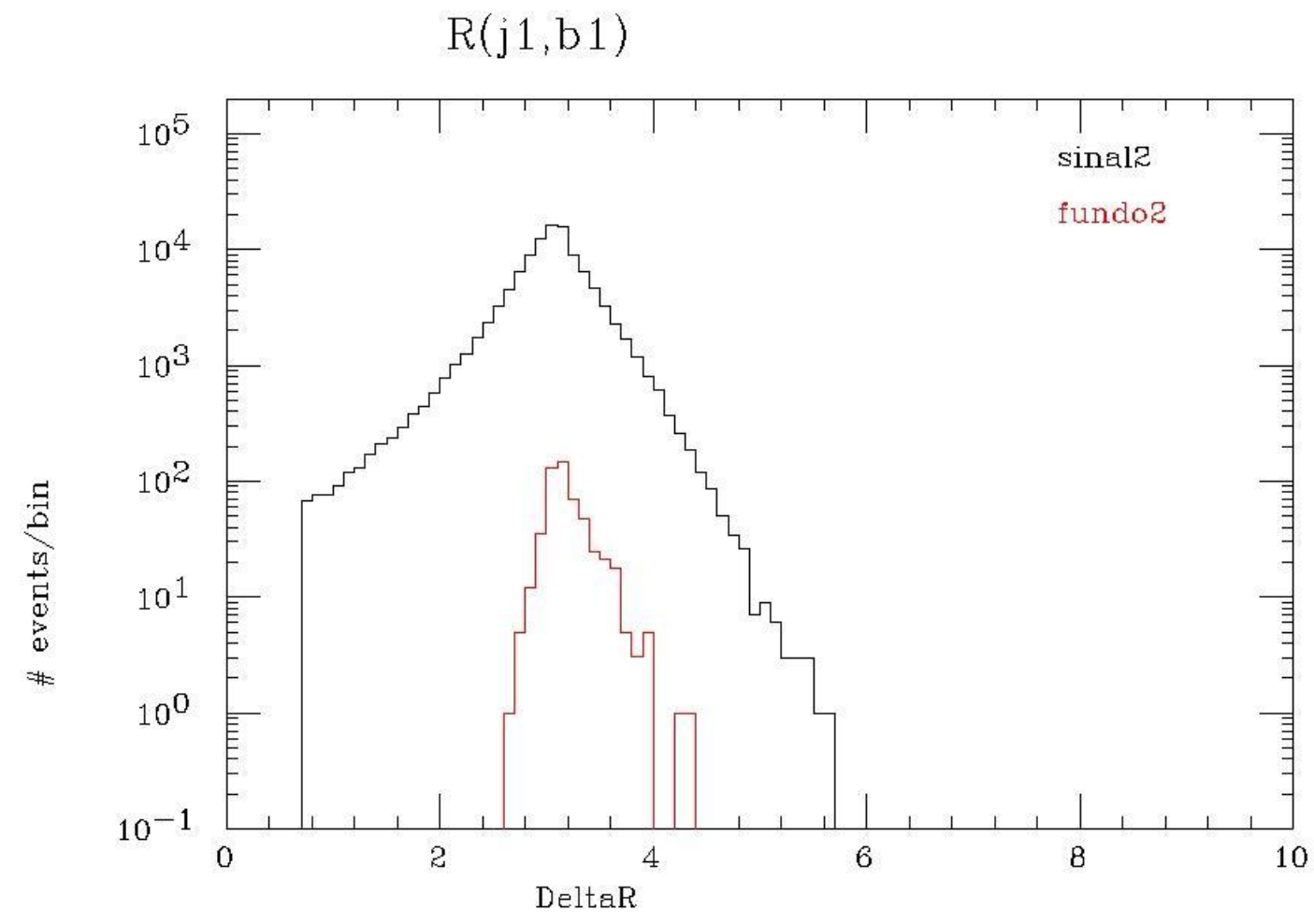

Figura 5.17: $\Delta R_{j 1 b 1}, p_{T}(b 1)>500 \mathrm{GeV}, \Delta R_{j 1 b 1}>0,7$.

Nesta nova distribuição de Delta R (j1,b1) percebe-se o corte removendo tanto eventos do sinal quanto do fundo. O pico que ocorre para valores mais altos de Delta $\mathrm{R}$ (para o fundo) permanece, assim como o pico para o sinal. Retomando a interpretação em termos do decaimento da figura 5.4, eliminase grande parte dos eventos com a característica de o jato mais energético de $b$ estar (predominantemente) numa direção próxima à do jato mais energético não proveniente de $b$, característica ausente no sinal, mas presente no fundo. 


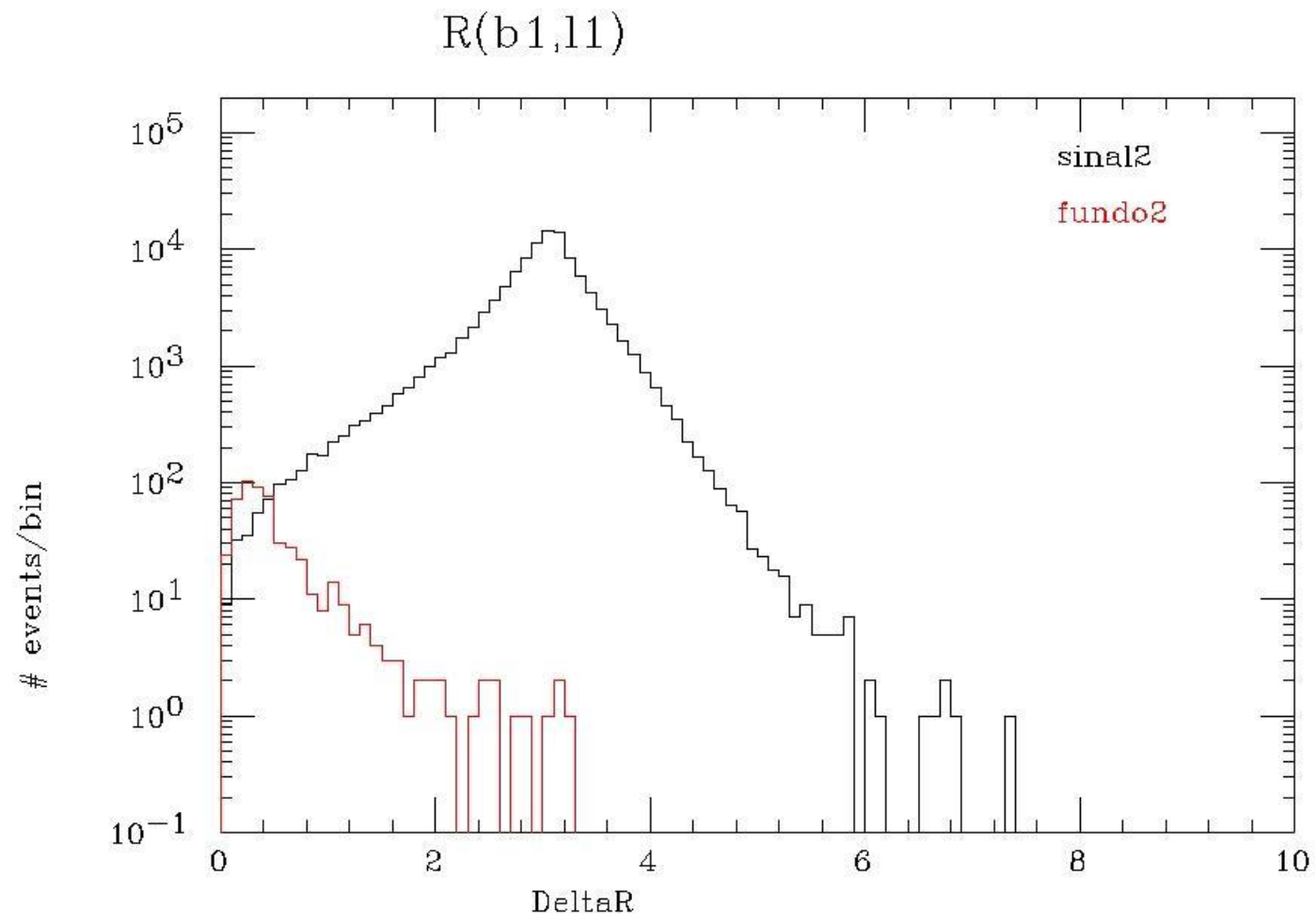

Figura 5.18: $\Delta R_{b 1 l 1}, p_{T}(b 1)>500 \mathrm{GeV}, \Delta R_{j 1 b 1}>0,7$.

Pode ser observado que nesta nova distribuição de Delta $\mathrm{R}(\mathrm{b} 1,11)$ o pico permanece, na distribuição do fundo, para valores pequenos de Delta $\mathrm{R}$, enquanto o outro pico foi eliminado pelo corte. A permanência deste pico sugere um corte final. Nota-se a consistência da interpretação em termos da direção dos jatos: após a eliminação dos eventos com o jato mais energético de $b$ na direção do jato mais energético (não originário de $b$ ), restam, para o fundo, eventos onde o jato mais energético de $b$ está na direção do lépton, portanto $o$ pico remanescente do fundo está em Delta $R \sim 0$. Esta característica está ausente no sinal, onde o jato de $b$ mais energético está (predominantemente) afastado tanto do jato mais energético (não originário de b) quanto do lépton. 


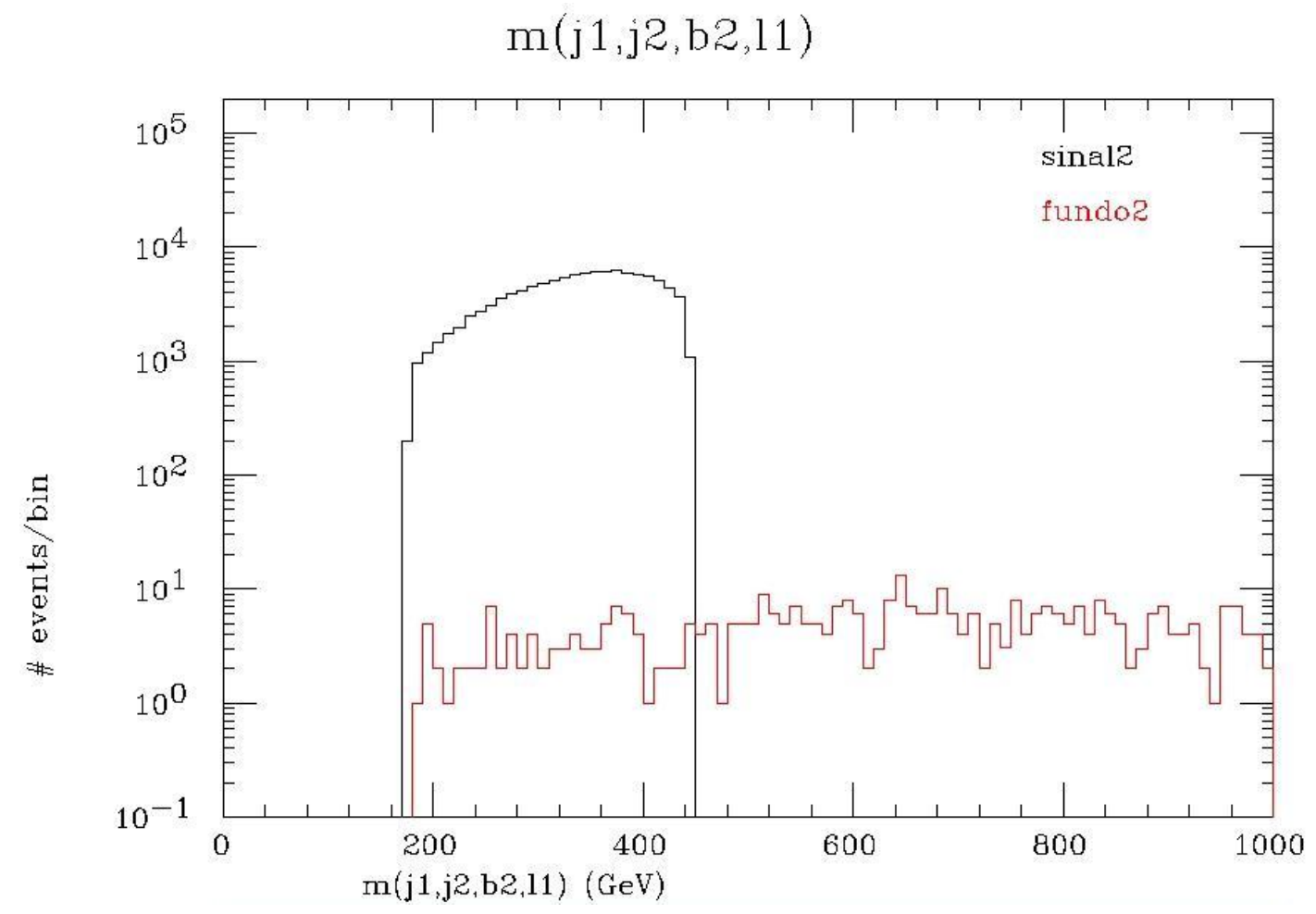

Figura 5.19: $m(j 1, j 2, b 2, l 1), p_{T}(b 1)>500 \mathrm{GeV}, \Delta R_{j 1 b 1}>0,7$.

Nota-se a redução pela metade do número de eventos do fundo $\sim 5 \times 10^{2}(527)$ eventos restantes, sem perda relevante de eventos do sinal $\sim 10^{5}$ (108182) eventos restantes.

Finalizando os cortes para este processo, utiliza-se $\Delta R_{b 1 l 1}>0,8$, para reduzir o pico remanescente do fundo, no gráfico 5.18. As figuras 5.20, 5.21, $5.22,5.23$ e 5.24 mostram os gráficos resultantes para o conjunto de cortes determinado neste estudo, para viabilizar a observação experimental do processo $p p \rightarrow d_{4} \bar{b}$. 


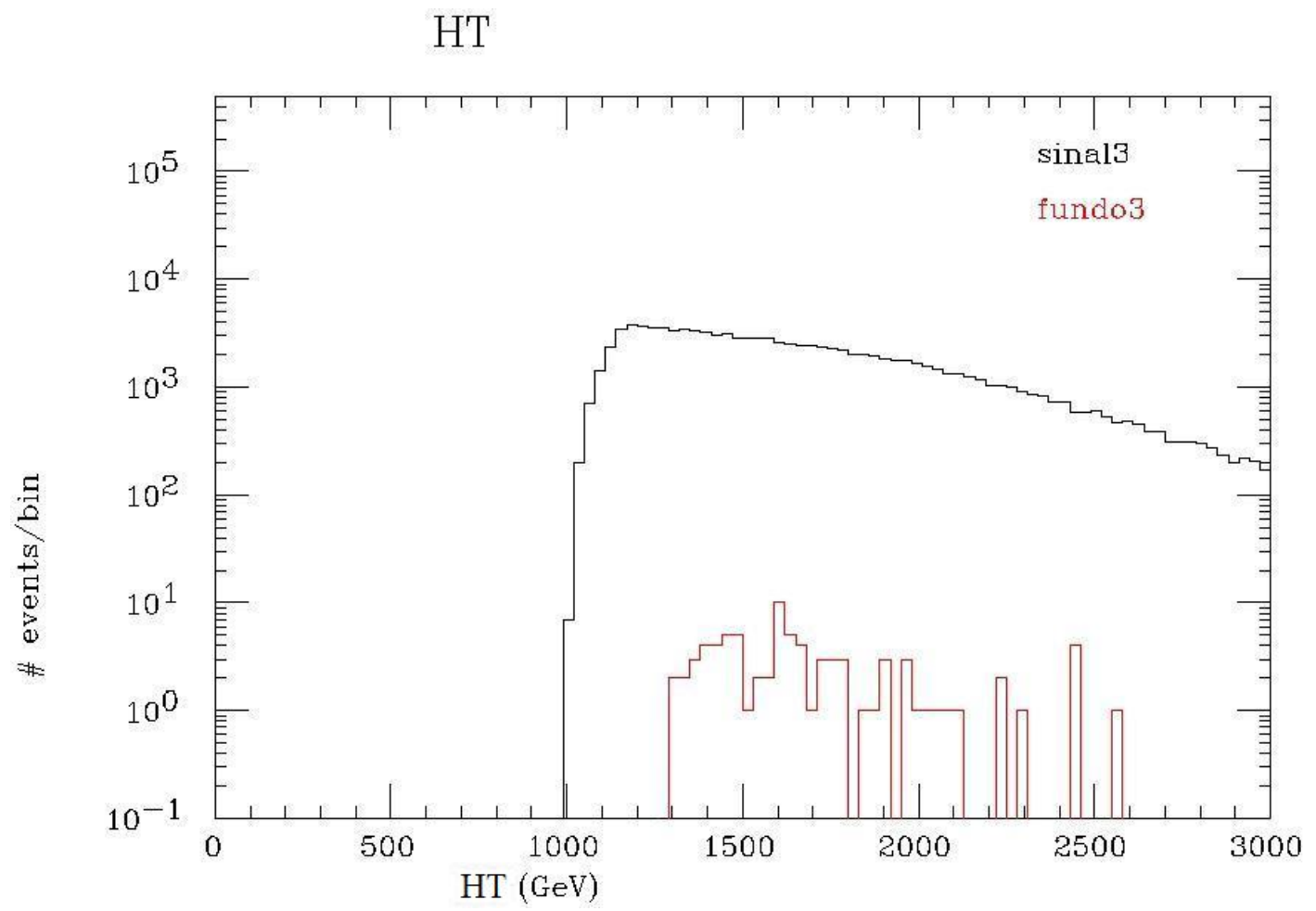

Figura 5.20: $H_{T}, p_{T}(b 1)>500 \mathrm{GeV}, \Delta R_{j 1 b 1}>0,7, \Delta R_{b 1 l 1}>0,8$.

Observa-se a redução ainda mais drástica do número de eventos do fundo e perdas desprezíveis de eventos do sinal, característica desejável para o conjunto de cortes. 


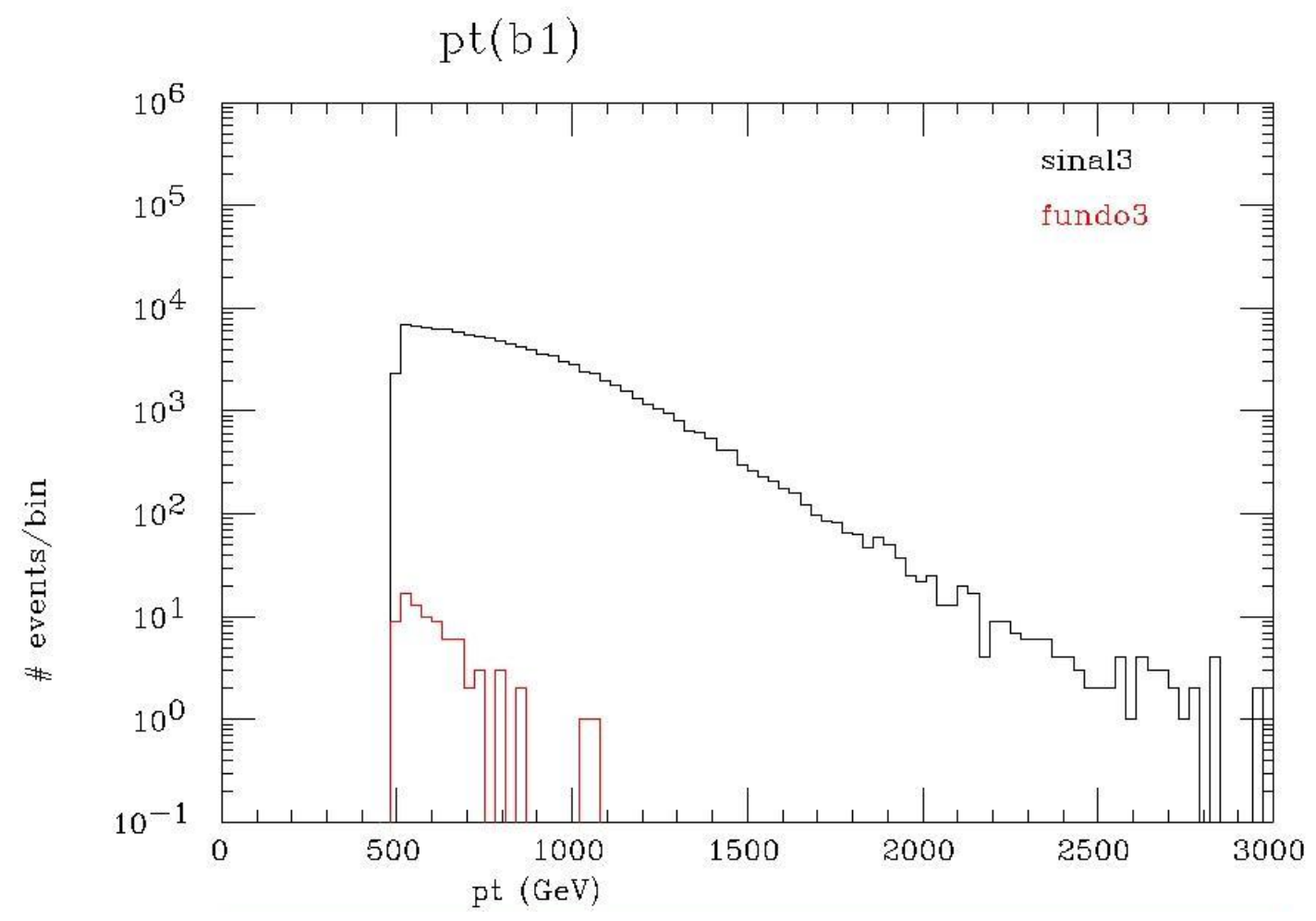

Figura 5.21: $p_{T}(b 1), p_{T}(b 1)>500 \mathrm{GeV}, \Delta R_{j 1 b 1}>0,7, \Delta R_{b 1 l 1}>0,8$.

Da mesma maneira, observa-se o fundo muito reduzido, mostrando que o conjunto de cortes serviu ao objetivo de otimizar o sinal com respeito ao fundo. 


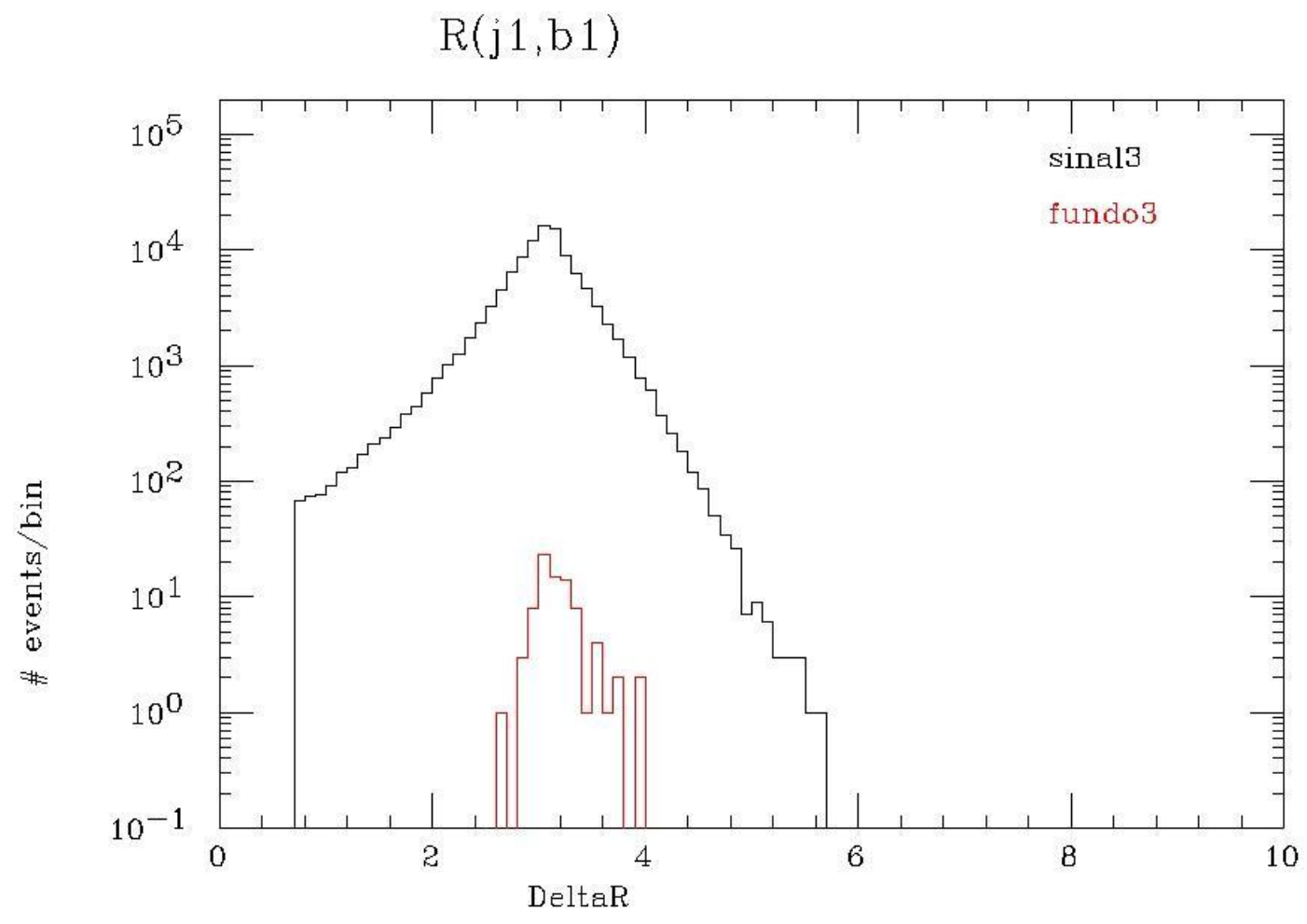

Figura 5.22: $\Delta R_{j 1 b 1}, p_{T}(b 1)>500 \mathrm{GeV}, \Delta R_{j 1 b 1}>0,7, \Delta R_{b 1 l 1}>0,8$.

Para evitar perdas excessivas de eventos do sinal, o pico central na distribuição do fundo foi reduzido mas não eliminado, mesmo assim resultando em estratégia de cortes bem sucedida. 


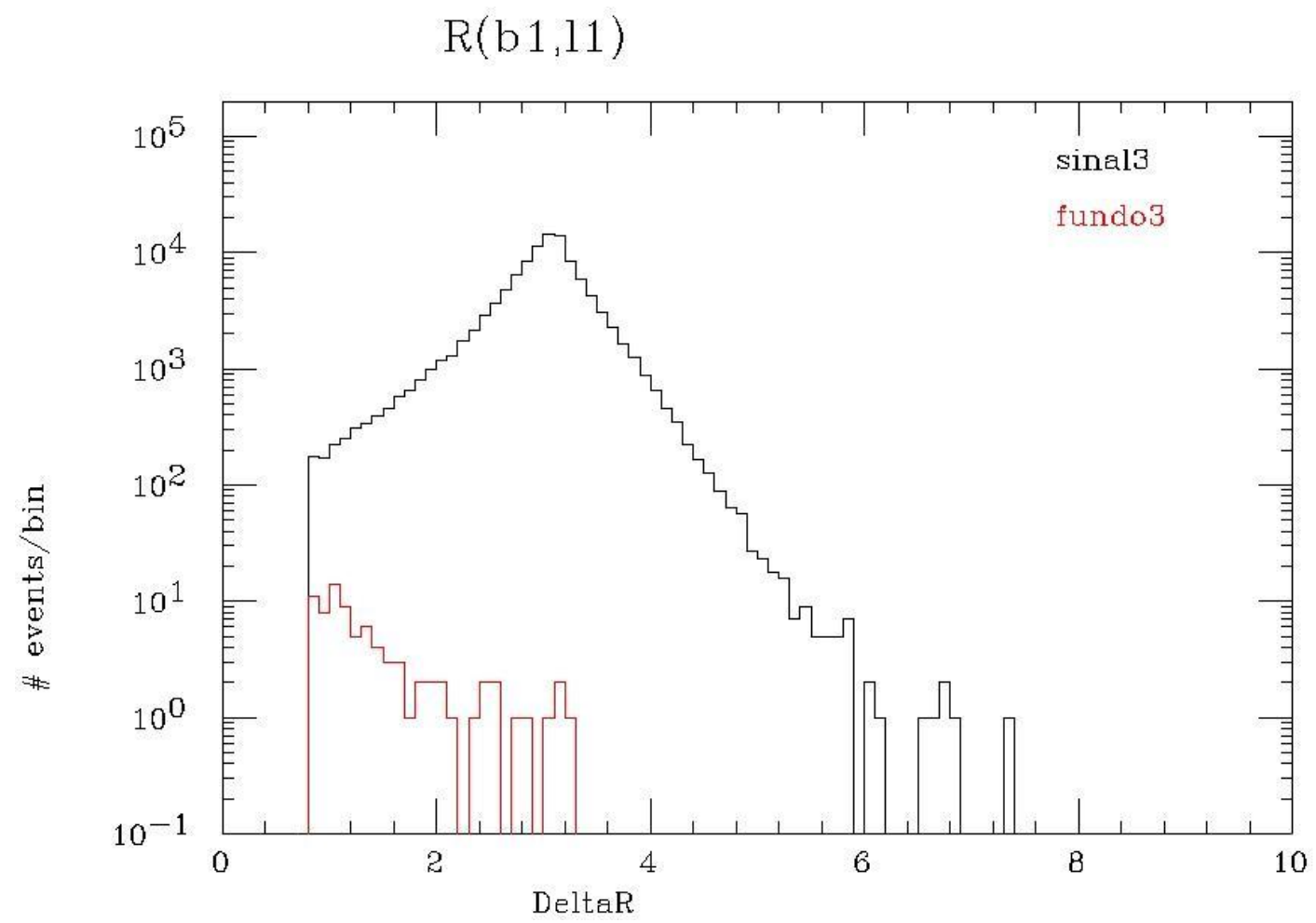

Figura 5.23: $\Delta R_{b 1 l 1}, p_{T}(b 1)>500 \mathrm{GeV}, \Delta R_{j 1 b 1}>0,7, \Delta R_{b 1 l 1}>0,8$.

Nota-se o efeito do último corte; eventos com $\Delta R_{b 1 l 1}<0,8$ não são incluídos. Observa-se também que o pico na distribuição do fundo foi reduzido mas, para evitar a demasiada perda de eventos do sinal, não foi completamente eliminado. 


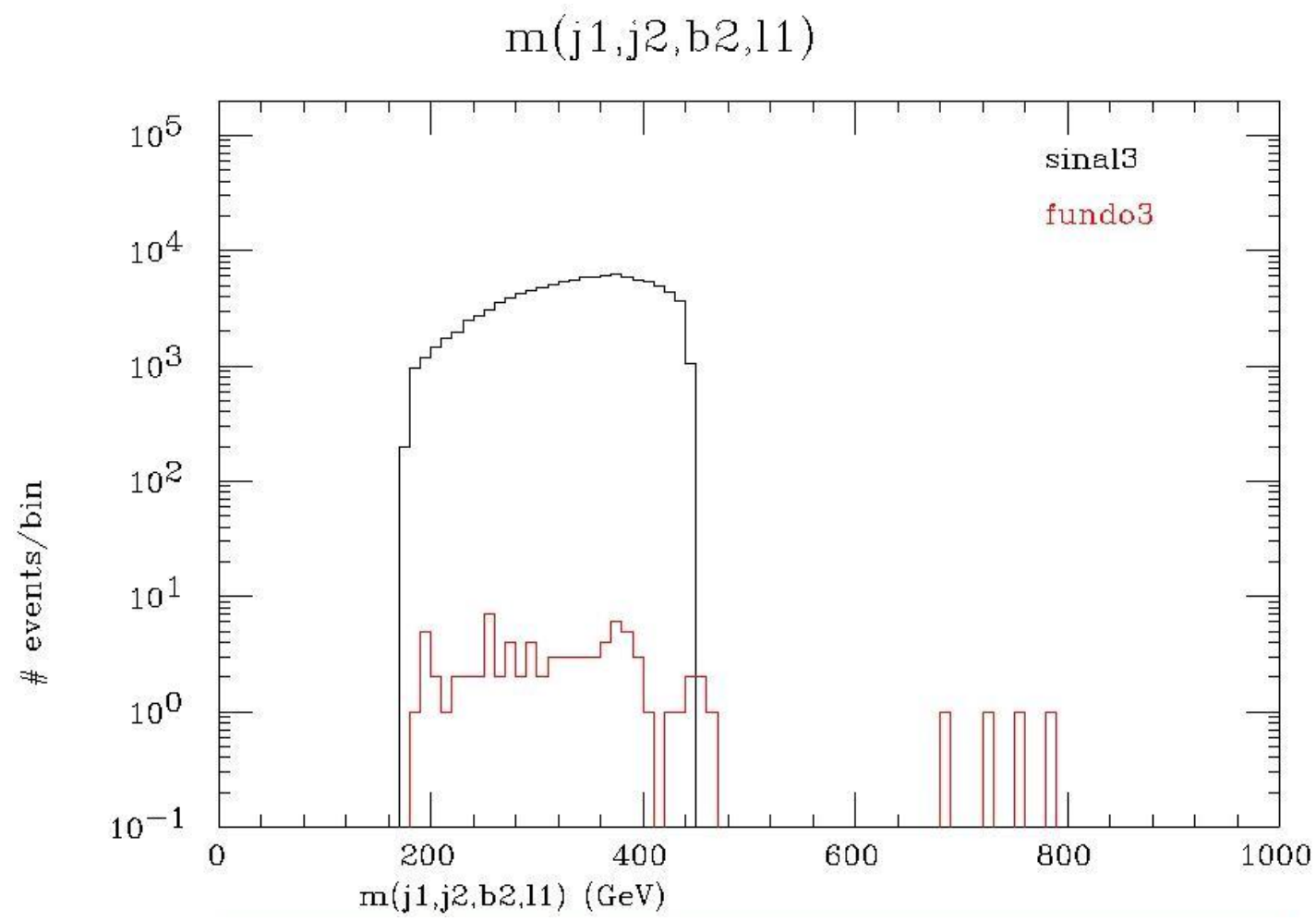

Figura 5.24: $m(j 1, j 2, b 2, l 1), p_{T}(b 1)>500 \mathrm{GeV}, \Delta R_{j 1 b 1}>0,7, \Delta R_{b 1 l 1}>$ 0,8 .

Para este corte final, pode ser verificada a característica do decaimento de $d_{4}$ pela massa invariante das quatro partículas, no caso do sinal, bem como a redução dos eventos do fundo.

Observa-se que restaram apenas $\sim 100$ eventos no fundo $(82)$ e $\sim 100$ mil eventos do sinal (107849), ou seja, o que resta do sinal é cerca de $10^{3}$ vezes a quantidade de eventos do fundo. Há grande diferença nas seções de choque, cuja razão entre fundo e sinal é de $10^{4}$ e foram gerados 10 vezes mais eventos para o fundo do que para o sinal.

Seja definido o fator de rejeição pela razão entre o número de eventos que restam após a aplicação de cortes e o número de eventos iniciais:

$$
r_{N}=\frac{N}{N_{0}}
$$


Então, com a aplicação do conjunto de cortes: $p_{T}(b 1)>500 \mathrm{GeV}$, $\Delta R_{j 1 b 1}>0,7$ e $\Delta R_{b 1 l 1}>0,8$, além do corte inicial aplicado sobre o momento transverso de $b$ 's $\left(p_{T}>30 \mathrm{GeV}\right)$, os fatores de rejeição para sinal e fundo são, respectivamente:

$$
r_{S}=0,53 \approx 0,5
$$

e

$$
r_{B}=4,1 \times 10^{-5} \approx 4 \times 10^{-5}
$$

O número de eventos se relaciona à seção de choque e à luminosidade por:

$$
N=\sigma L
$$

Para gerar o número inicial de eventos do fundo, a luminosidade necessária é:

$$
L_{b}=\frac{2 \times 10^{6}}{5,4 \times 10^{4}} \mathrm{fb}^{-1} \approx 37 \mathrm{fb}^{-1}
$$

e para o sinal

$$
L_{s}=\frac{2 \times 10^{5}}{5,4} \mathrm{fb}^{-1} \approx 37 \times 10^{3} \mathrm{fb}^{-1}
$$

Como a seção de choque não depende do número de eventos medidos e a luminosidade deve ser a mesma, para comparar sinal e fundo no mesmo gráfico, ou seja, comparando-se eventos para a mesma luminosidade, o número de eventos do sinal deve ser multiplicado por $10^{-3}$. Como restaram 82 eventos do fundo, o número de eventos do sinal para a mesma luminosidade do fundo é $S=108$. Portanto a razão entre o número de eventos do sinal e do fundo é:

$$
\frac{S}{B}=1,3
$$

A significância estatística, definida por:

$$
\rho=\frac{S}{\sqrt{S+B}}
$$

deve valer ao menos 5, para justificar a descoberta do processo em comparação com o fundo.

Seja a relação do número de eventos (do sinal ou do fundo) com a luminosidade, seção de choque do decaimento e fração de eventos após os cortes, 
relação que generaliza a equação 5.10:

$$
N=L \sigma_{N} r_{N} .
$$

A aplicação desta equação aos eventos do sinal e do fundo leva a:

$$
S=L \sigma_{S} r_{S}
$$

para o sinal e:

$$
B=L \sigma_{B} r_{B}
$$

para o fundo. Inserindo as duas últimas expressões na equação 5.11, resulta a luminosidade acumulada necessária como função da significância requerida:

$$
L=\frac{\rho^{2}}{\sigma_{S}^{2} r_{S}^{2}}\left(\sigma_{S} r_{S}+\sigma_{B} r_{B}\right) .
$$

Com os resultados da tabela 10 e as equações (5.8) e (5.9), requerendo-se significância $\rho=5$, para se confirmar eventos deste processo no LHC, será necessária luminosidade acumulada de $L_{c} \approx 15 \mathrm{fb}^{-1}$, com o LHC funcionando com $14 \mathrm{TeV}$ de energia no centro de massa.

Fica confirmada então a viabilidade da verificação experimental do processo, com a estratégia de cortes acima descrita.

Pode ser estimada também a viabilidade de deteç̧ão do processo a $7 \mathrm{TeV}$. Por simulação, são determinadas as seções de choque para os decaimentos dos processos do sinal e do fundo a $7 \mathrm{TeV}$ :

$$
\begin{gathered}
\sigma_{S}^{\prime}=0,76 \mathrm{fb} \\
\sigma_{B}^{\prime}=9,4 \times 10^{3} \mathrm{fb}
\end{gathered}
$$

Após a aplicação do principal corte obtido para o caso de $14 \mathrm{TeV}$, ou seja $p_{T}(b 1)>500 \mathrm{GeV}$, resultam os fatores de rejeição para sinal e fundo, respectivamente:

$$
\begin{gathered}
r_{S}^{\prime}=0,31 \\
r_{B}^{\prime}=1,2 \times 10^{-4} .
\end{gathered}
$$

Com o uso da equação 5.15, segue que, para se detectar eventos deste processo, a $7 \mathrm{TeV}$ no LHC, será necessária uma luminosidade de aproximadamente $L^{\prime}=605 \mathrm{fb}^{-1}$, sendo, portanto, inviável a observação de $p p \rightarrow d_{4} \bar{b}$ no LHC a $7 \mathrm{TeV}$, requerendo-se seu funcionamento a $14 \mathrm{TeV}$ para observação do processo. 


\subsection{Detecção de $p p \rightarrow u_{4} \bar{t}$}

A vantagem da busca por este processo, comparado com o anterior é que, neste, utiliza-se o fato de o acoplamento entre o quark $t$ e o primeiro modo de Kaluza-Klein do glúon $\left(G_{1}\right)$ ser maior do que o acoplamento do quark $b \mathrm{com}$ $G_{1}$, aumentando significativamente a seção de choque do sinal, sendo que a seção de choque do fundo permanece praticamente inalterada. A principal desvantagem é que, nos decaimentos decorrentes, a topologia e o estado final são idênticos aos do fundo, não havendo, como havia no caso anterior, alguma partícula muito energética que diferencie o fundo do sinal.

\subsubsection{Decaimento}

Considera-se o decaimento $u_{4} \rightarrow b W^{+}$, por ser o único apreciável, uma vez que o vértice envolve a quarta linha da matriz CKM aumentada, da qual apenas $V_{44}$ e $V_{43}$ são relevantes, dos quais o primeiro é considerado apenas no decaimento $u_{4} \rightarrow d_{4} W^{+}$que é suprimido cinematicamente $\left(m_{u 4}<\right.$ $\left.m_{d 4}+m_{W}\right)$.

Novamente, foi exigido um lépton carregado no decaimento, com $W^{+}$ decaindo em pósitron e neutrino. O pósitron indica que o lépton provém de decaimento de $u_{4}$. A consideração do múon dobra os valores de seções de choque aqui apresentados. Processos envolvendo o $\tau$ não foram considerados, pois resultariam em decaimentos que tornariam complicada sua identificação. O decaimento relevante para o $\bar{t}$ é em $\bar{b}$, por razão análoga ao decaimento de $u_{4}$.

Dentre os possíveis produtos de decaimento para o processo, a figura 5.25 mostra o diagrama de Feynman para o processo final presentemente estudado.

O principal fundo identificado é mostrado na figura 5.26 e, comparação com a figura 5.25, mostra que a topologia dos dois decaimentos é a mesma, dificultando a separação de fundo e sinal.

Os pacotes MadEvent + Bridge foram utilizados, como anteriormente, para decair as partículas do processo. As seções de choque, para sinal e fundo decaídos, são mostradas na tabela 11, para $14 \mathrm{TeV}$ no centro de massa. Foram gerados, tanto para o sinal quanto para o fundo, $10^{5}$ eventos. Observa- 


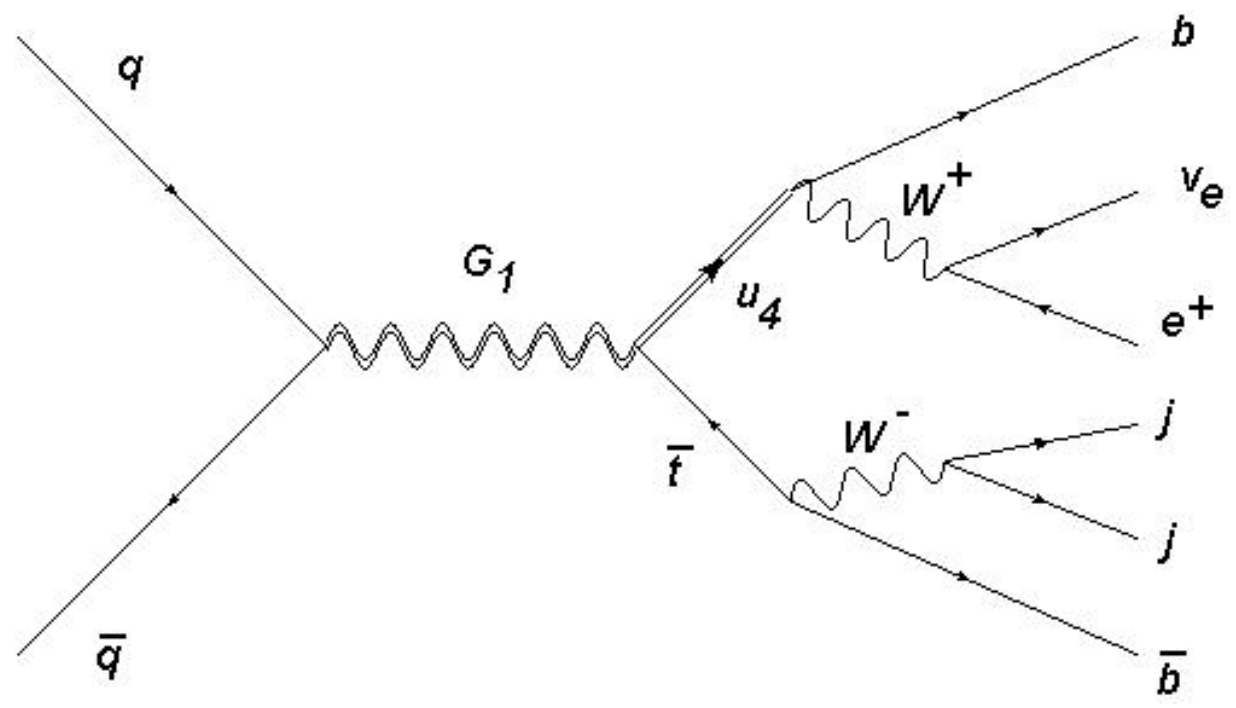

Figura 5.25: Diagrama de Feynman de canal de decaimento para o processo $q \bar{q} \rightarrow u_{4} \bar{t}$.

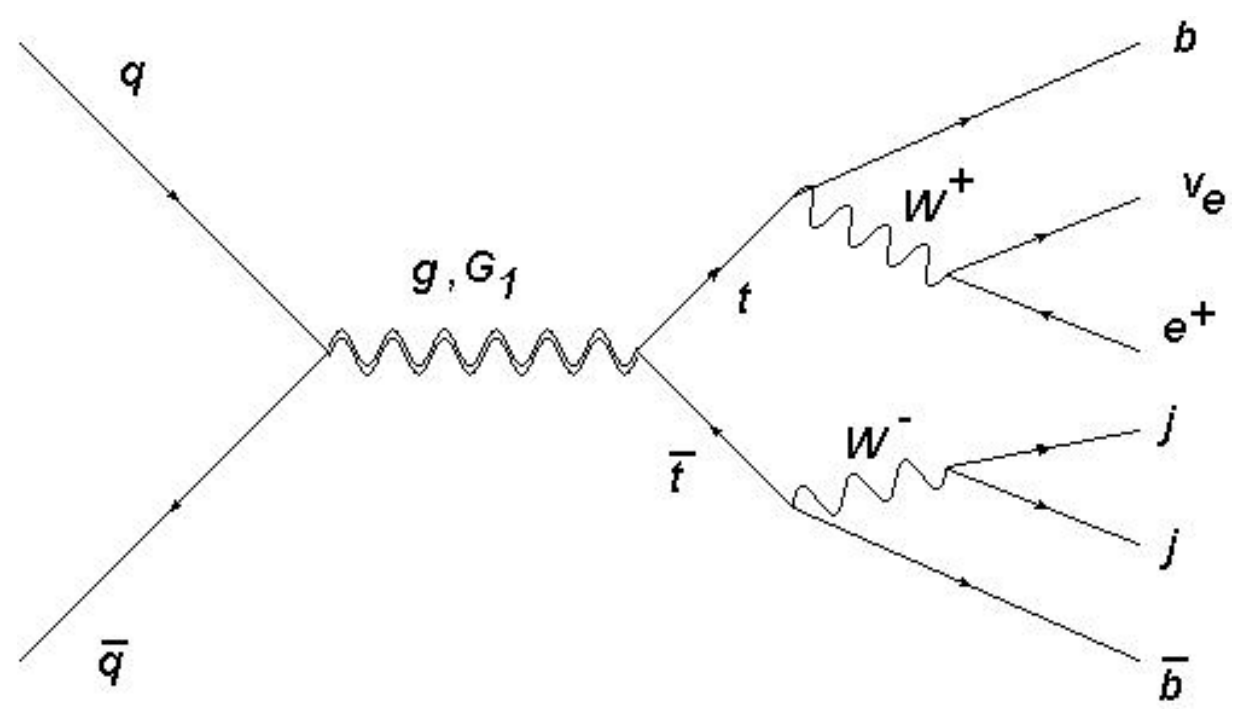

Figura 5.26: Diagrama de Feynman de canal de decaimento para $t \bar{t}$, como fundo para $u_{4} \bar{t}$.

se que a seção de choque é cerca de 3 vezes maior do que para o decaimento anterior, decorrente de $p p \rightarrow d_{4} \bar{b}$. Vale enfatizar que, para definição do caso a ser tratado, a análise e o estudo dos cortes foram especializados para o caso 
no qual $m_{u 4}=500 \mathrm{GeV}, m_{1}=2 \mathrm{TeV}$ e para a energia de $14 \mathrm{TeV}$ no centro de massa.

Tabela 11 - Seções de choque (em fb) para sinal e fundo decaídos de

$$
p p \rightarrow u_{4} \bar{t} ; E_{C M}=14 \mathrm{TeV}
$$

\begin{tabular}{|c|c|}
\hline sinal & fundo \\
\hline $1,7.10^{-2}$ & $5,4.10^{1}$ \\
\hline
\end{tabular}

\subsubsection{Análise e cortes}

Os gráficos 5.27, 5.28, 5.29 e 5.30 mostram, respectivamente, $H_{T}, p_{T}(b 1)$, $\Delta R_{j 1 b 1}$ e $m(b 1, l 1)$, antes de qualquer corte.

\section{HT}

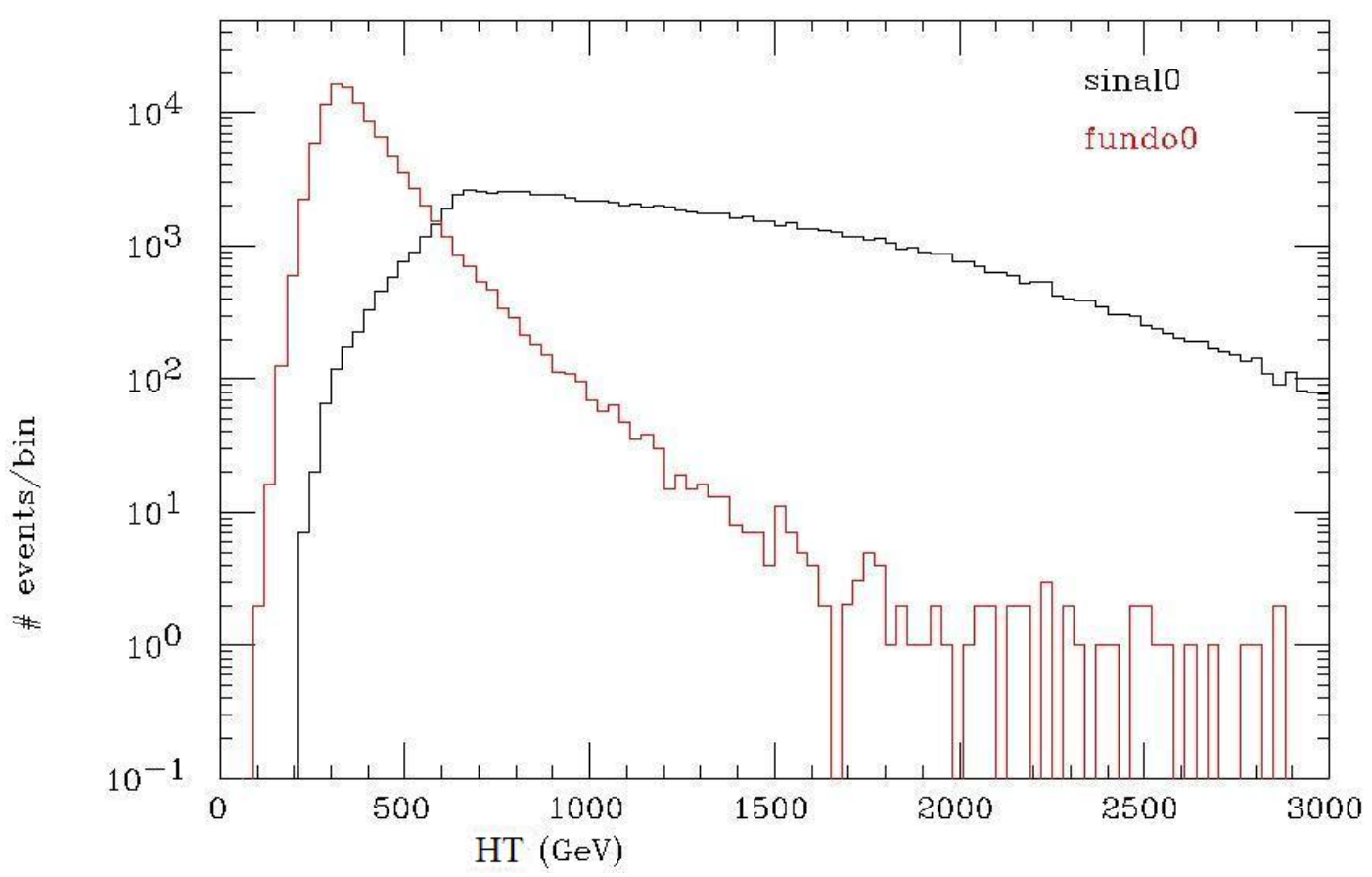

Figura 5.27: $H_{T}$ antes de corte.

O gráfico mostra um pico na distribuição do fundo para valores pequenos de $H_{T}$, enquanto na distribuição do sinal o pico está ausente. 


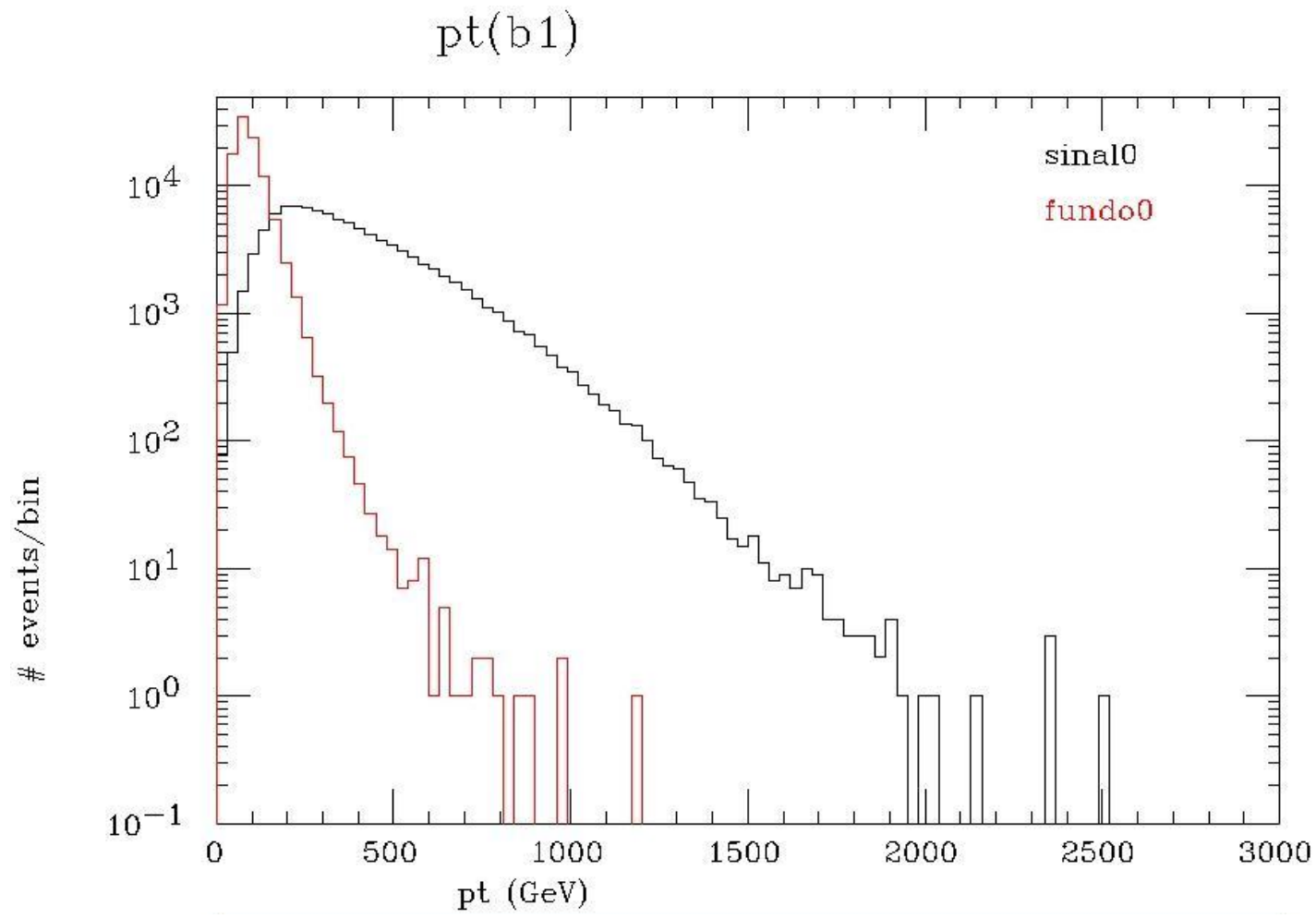

Figura 5.28: $p_{T}(b 1)$ antes de corte.

Novamente a distribuição do fundo apresenta um pico, agora para pequenos valores de $p_{T}(b 1)$. O pico é mais acentuado do que o pico do sinal e devido à grande quantidade de eventos com pequeno $p_{T}$ para o fundo, a distribuição sugere um corte onde muitos eventos do fundo serão eliminados. 


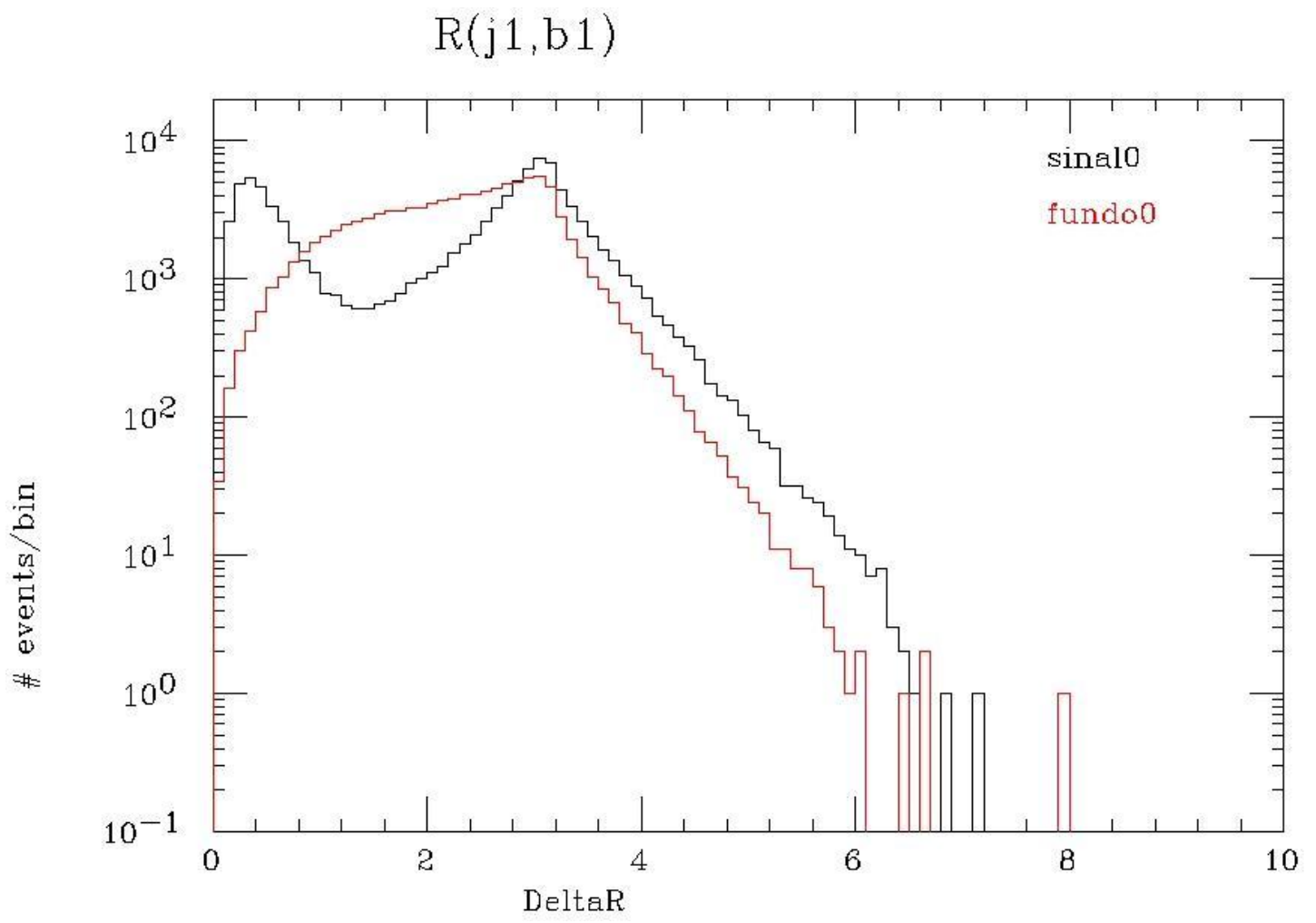

Figura 5.29: $\Delta R_{j 1 b 1}$, antes de corte.

Os gráficos de Delta R para sinal e para fundo são distintos, com o sinal apresentanto dois picos e o fundo apenas um. Contudo, devido à grande sobreposição entre as distribuições, não há possibilidade de corte eficaz neste gráfico. 


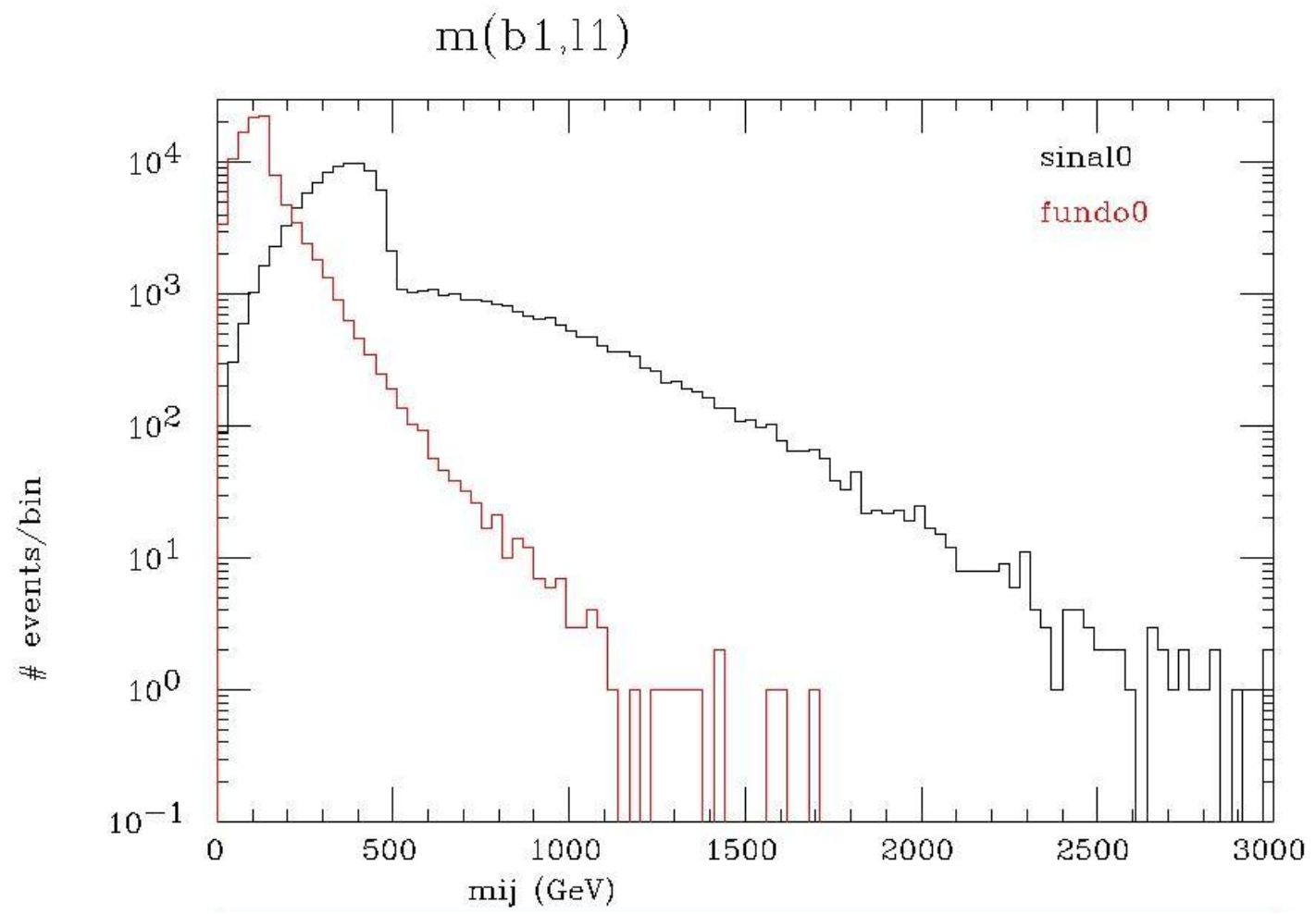

Figura 5.30: $m(b 1, l 1)$ antes de corte.

Os limites das distribuições para sinal e fundo mostram claramente que o bottom e o pósitron têm origens distintas nos dois casos; para o fundo as duas partículas têm origem no quark top e para o sinal a origem é o quark $u_{4}$, que é mais massivo. Este é outro fato distintivo para sinal e fundo, que possibilitaria a identificação da quarta geração de quarks.

Com base no estudo do processo anterior, pois o fundo é similar, o corte aplicado é tal que apenas eventos com $p_{T}(b 1)>500 \mathrm{GeV}$ foram considerados. Observa-se o resultado nas figuras 5.31, 5.32, 5.33 e 5.34 . 


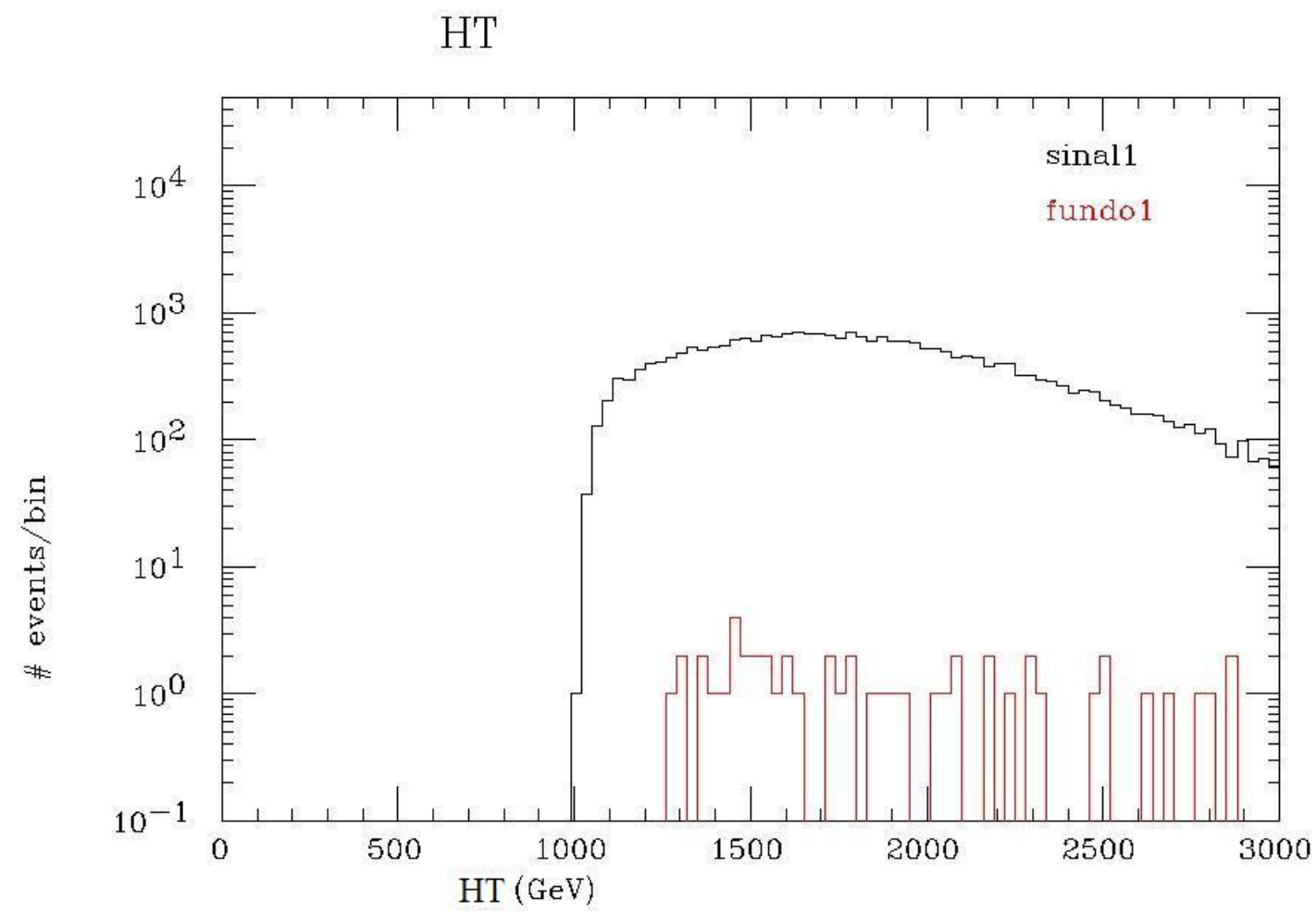

Figura 5.31: $H_{T}, p_{T}(b 1)>500 \mathrm{GeV}$.

Observa-se que grande quantidade de eventos do sinal permaneceu (aproximadamente um quarto), enquanto o fundo ficou muito reduzido, restando apenas uma fração de cerca de $5 \times 10^{-4}$ de seus eventos. 


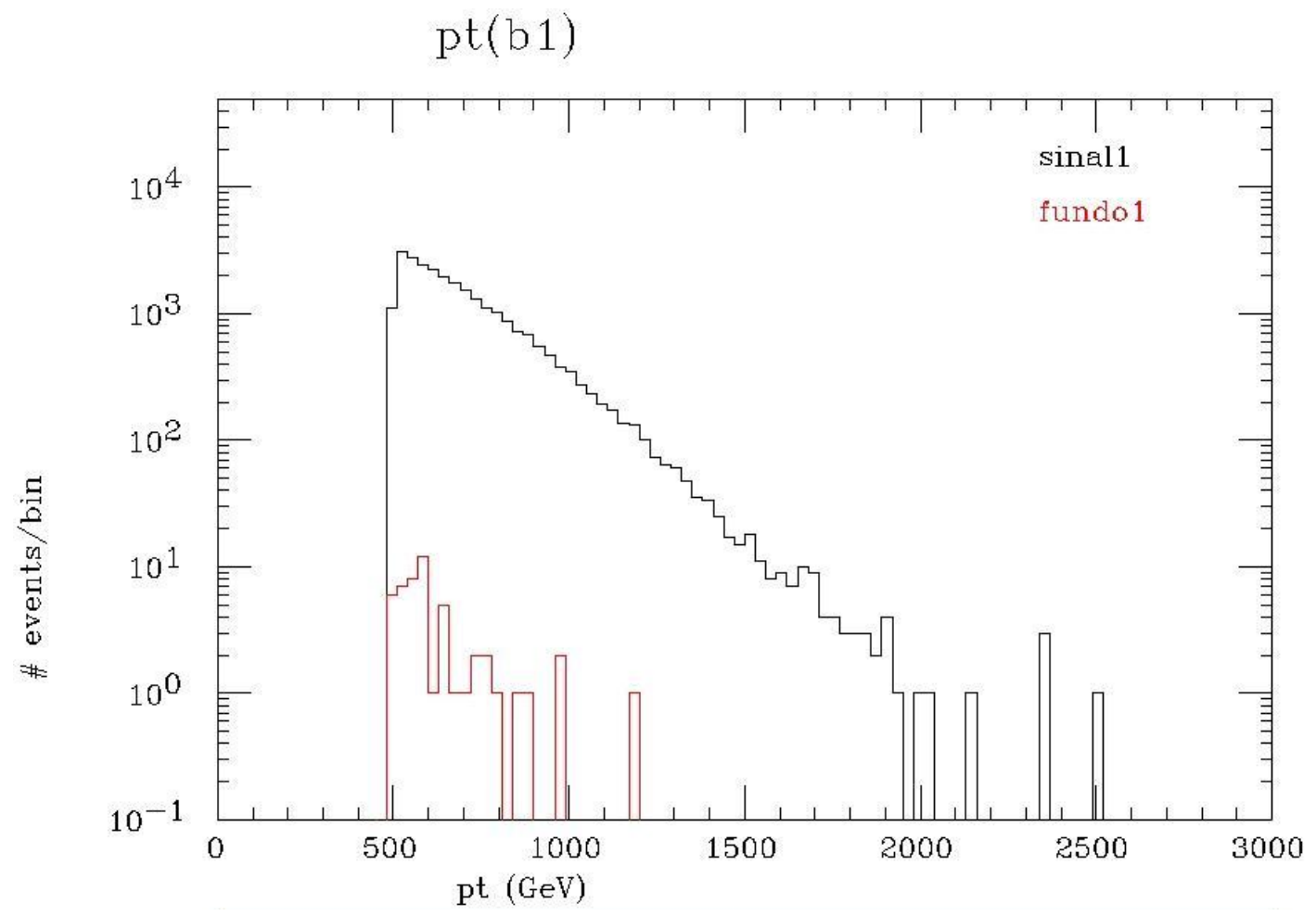

Figura 5.32: $p_{T}(b 1), p_{T}(b 1)>500 \mathrm{GeV}$.

Nota-se o corte aplicado e a drástica redução do fundo. 


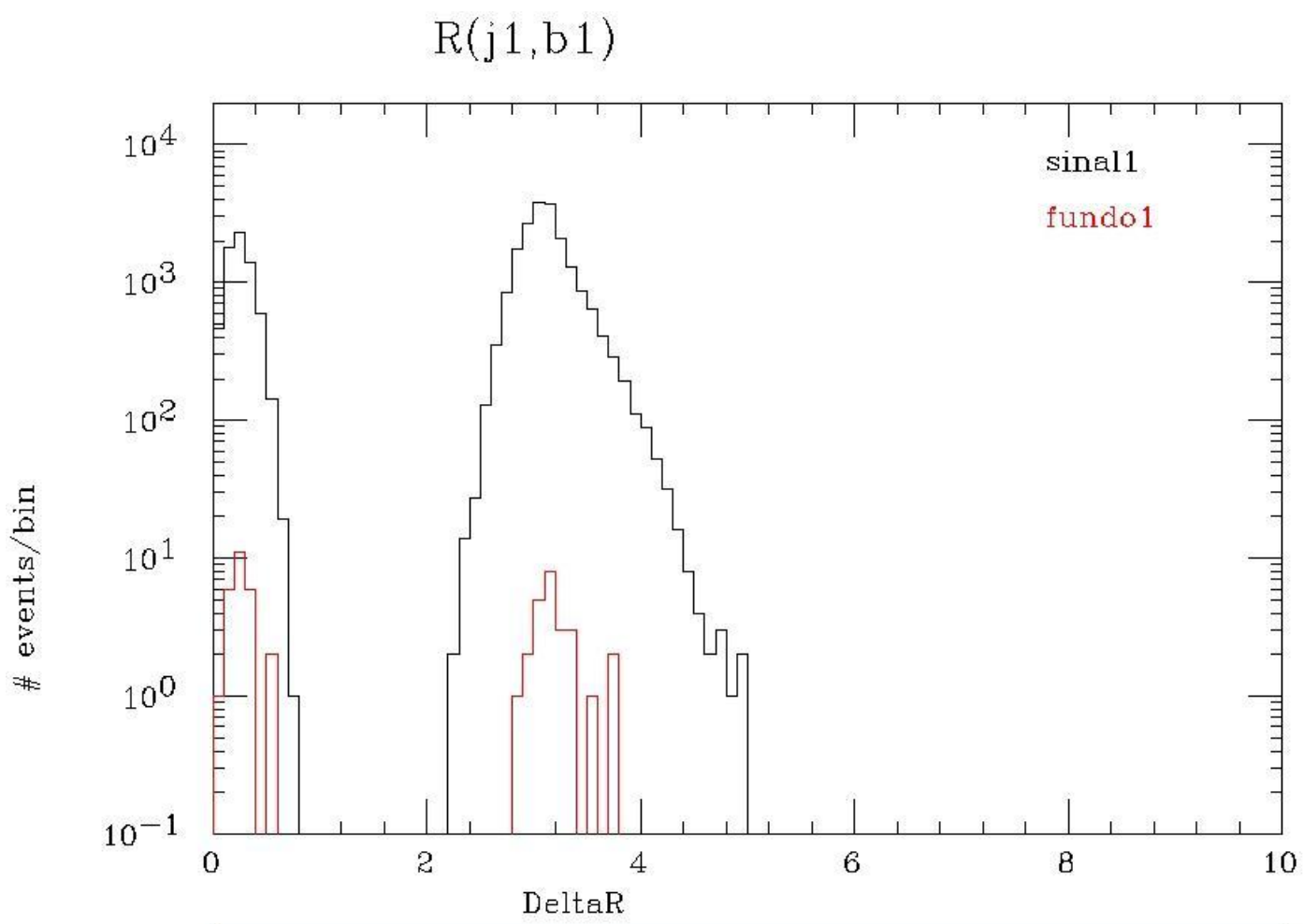

Figura 5.33: $\Delta R_{j 1 b 1}, p_{T}(b 1)>500 \mathrm{GeV}$.

Neste gráfico observa-se, nas duas distribuições, dois picos desconexos em cada e a forma de ambas é similar (refletindo a idêntica topologia dos decaimentos), com a distribuição do fundo muito menor do que a do sinal. Não oferece boas possibilidades de cortes. 


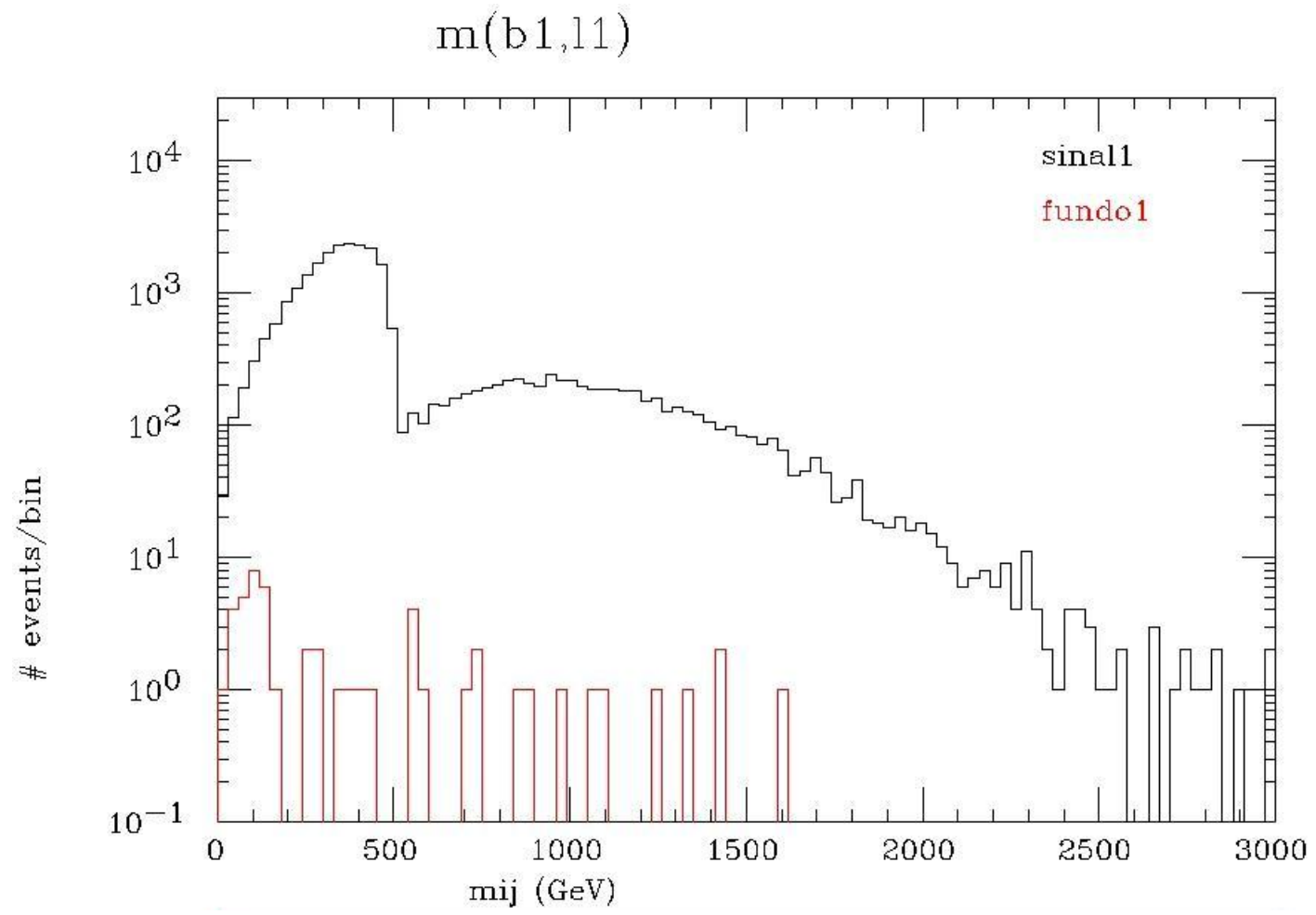

Figura 5.34: $m(b 1, l 1), p_{T}(b 1)>500 \mathrm{GeV}$.

Graças à permanência de grande quantidade de eventos do sinal, a massa invariante de $b 1$ e $e^{+}$continua denunciando a origem comum destas partículas como sendo o $u_{4}$. A grande redução de eventos do fundo não mais permite identificar a origem das duas partículas, neste caso.

Vale destacar que dos $N_{0}=10^{5}$ eventos presentes inicialmente no sinal e no fundo, com o corte $p_{T}(b 1)>500 \mathrm{GeV}$, restaram cerca de 25 mil (26075) eventos para o sinal e 51 eventos para o fundo. Estes resultados implicam nos fatores de rejeição para sinal e fundo, respectivamente:

$$
\begin{gathered}
r_{S}=0,26 \\
r_{B}=5,1 \times 10^{-4}
\end{gathered}
$$

Outro fator notável é a distribuição de $\Delta R_{j 1 b 1}$, onde verifica-se que, mesmo com o fundo reduzido, ambos os gráficos (sinal e fundo) apresentam dois picos desconexos. Isto se deve a eventos com o jato (não b-jet) 
mais energético e o jato de bottom mais energético ora na mesma direção $(\Delta R \sim 0)$, ora em direções opostas $(\Delta R \sim \pi)$ considerando-se o corte, tanto para sinal quanto para o fundo, o que reflete a topologia idêntica dos decaimentos para sinal e fundo.

Por fim, uma maneira de identificar este sinal característico é observar o limite para a massa invariante entre $b$ e $l$. Essa massa tem limite de 500 $\mathrm{GeV}$ para o sinal (massa de $u_{4}$ ) enquanto o limite é de $174 \mathrm{GeV}$ para o fundo (massa do top). O fato de haver eventos após o limite é devido a, por vezes, o bottom mais energético provir de $\bar{t}$ e não de $u_{4}$ (caso do sinal) ou o bottom mais energético não ser gerado a partir do mesmo top que o lépton (caso do fundo). O fato de as distribuições entre massa invariante de $b 1$ e $l$ serem distintas, para este sinal e seu fundo, pode ser verificado pelos experimentalistas, como característica deste sinal.

Considerando ainda o número de eventos, podem ser analisadas as seções de choque para o sinal e para o fundo. Para serem observados $10^{5}$ eventos do fundo, é necessário uma luminosidade de:

$$
L_{f}=\frac{10^{5}}{5,4 \times 10^{4}} \mathrm{fb}^{-1}=1,8 \mathrm{fb}^{-1}
$$

e para o sinal:

$$
L_{s}=\frac{10^{5}}{16,8} \mathrm{fb}^{-1}=5,9 \times 10^{3} \mathrm{fb}^{-1} .
$$

Para comparação dos números de eventos entre sinal e fundo, deve ser levado em consideração que a luminosidade será a mesma para ambos, o que significa que, para comparação dos processos no mesmo gráfico, o número de eventos do sinal (antes e depois dos cortes) deve ser multiplicado por $3,090 \times 10^{-4}$.

Desta maneira nota-se que, após os cortes, a razão de eventos observados do sinal com respeito ao fundo, passa a ser:

$$
\frac{26075}{51} \times 3,090 \times 10^{-4}=0,16 .
$$

Com o uso da equação 5.15 verifica-se que, para se obter significância $\rho=5$, confirmando-se a observação do processo, é necessária luminosidade acumulada de $L_{c} \approx 42 \mathrm{fb}^{-1}$ a $14 \mathrm{TeV}$, ficando demonstrada a observabilidade 
do processo no LHC. O processo possui ainda características distintivas como o limite para a massa invariante de $b 1$ e $l 1$.

Em resumo, tanto o processo $p p \rightarrow d_{4} \bar{b}$ quanto o processo $p p \rightarrow u_{4} \bar{t}$ poderão ser observados no LHC com as estratégias de cortes aqui apresentadas. 


\section{Capítulo 6}

\section{Conclusão}

O objetivo desta tese é mostrar a viabilidade da contrução e observação experimental de modelos da quebra da simetria eletrofraca e das massas dos férmions, por meio de acoplamentos fortes na escala TeV, através o estudo de sua fenomenologia. Algumas extensões do Modelo Padrão (seção 2.4) que resolvem o problema da hierarquia são mencionadas, tendo em vista possíveis extensões deste trabalho com a inclusão de ideias destas extensões.

Dentre as extensões possíveis do Modelo Padrão, trabalha-se aqui com um espaço com uma dimensão extra curva compactificada em orbifold (capítulo 3), como realização de teoria com acoplamentos que são fortes, mas não com todas as gerações de férmions (acoplamentos não-universais), possibilitando a condensação de quarks de uma quarta geração. Assim, é introduzido o modelo de Randall-Sundrum [4]. O trabalho segue com a descrição e estudo de campos escalares, espinoriais e vetoriais no espaço estendido, incluindo a determinação das "funções de onda"dos campos em cinco dimensões. O problema da hierarquia é resolvido (seção 3.3), neste modelo modificado, com a localização da função de onda do bóson de Higgs próxima a um dos pontos fixos do espaço, a brana $\mathrm{TeV}$, enquanto os demais campos se propagam por toda a dimensão extra.

Para que os campos estendidos à quinta dimensão realizem a extensão do Modelo Padrão (MP), é necessário estender o grupo de simetria do MP, de forma a adequar o modelo estendido aos vínculos de precisão eletrofracos. Além disso, é necessário caracterizar as funções de onda 5-dimensionais 
das partículas do MP, de forma que este seja obtido como caso limite da nova teoria a baixas energias. Para tanto é incluída uma quarta geração de férmions (capítulo 4), focando-se, neste trabalho, nos quarks, dos quais o quark do tipo up condensa e o condensado gera massa para todas as partículas. Nota-se que os acoplamentos nesta teoria não são universais, com o primeiro modo de Kaluza-Klein do glúon se acoplando fortemente à quarta geração de quarks e misturando sabor, apesar de ser uma partícula neutra. É também o responsável pela condensação dos quarks do tipo up da quarta geração.

Para a completa determinação do modelo que, a baixas energias, reduzse ao Modelo Padrão, foi necessário agregar ao modelo 5-dimensional, com o mecanismo de condensação, os valores de todas as constantes dos quarks (tabelas 2 e 3) com as condições de serem obtidas suas corretas massas e o setor experimentalmente conhecido da matriz CKM, constantes obtidas numericamente. Resulta desta parte do trabalho a matriz CKM estendida (equações 4.51 e 4.52), que possibilita cálculos de processos envolvendo a mistura de quarks da quarta geração.

Uma vez impostos esses vínculos sobre as constantes, resulta que, com uma estimativa para a massa do primeiro modo de Kaluza-Klein do glúon, são obtidos acoplamentos para todos os quarks, como função do acoplamento do modo zero do glúon (tabelas 4 e 5). Os acoplamentos obtidos permitiram o cálculo de processos envolvendo os quarks da quarta geração, possibilitando seu estudo fenomenológico (capítulo 5).

Os resultados fenomenológicos foram obtidos com base no modelo determinado no capítulo 4, utilizando-se os valores de constantes que determinam as funções de onda 5-dimensionais dos quarks e dos acoplamentos decorrentes. A obtenção dos resultados se deu por meio de simulações computacionais para processos que são muito distintivos de uma categoria de modelos, por apresentarem troca de sabor através de correntes neutras, característica que permite eliminar grande parte do fundo, originário da QCD, presente no LHC.

Foram estudados dois processos com potencial de observação no LHC (capítulo 5): $p p \rightarrow u_{4} \bar{t}$ e $p p \rightarrow d_{4} \bar{b}$. Os estudos incluem a determinação das seções de choque, por simulações computacionais, cujas partículas resultantes $\left(u_{4}, d_{4}\right.$ e $\left.\bar{t}\right)$ tiveram que ser, porteriormente, decaídas a estados finais 
observáveis experimentalmente. Estratégias de cortes tiveram que ser determinadas para a observação dos sinais contra fundos formidáveis da QCD que estarão presentes no acelerador.

Tal determinação, realizada nas seções 5.2 e 5.3, foi baseada nas distribuições de eventos como funções de grandezas convenientes (por exemplo, distribuição de eventos como função do momento transverso de uma partícula). Os cortes foram determinados pela observação de tais distribuições e pela busca da otimização do número de eventos do sinal com respeito ao número de eventos do fundo.

Segue então um estudo que busca estimar a viabilidade de observação de ambos os processos no LHC, após a aplicação dos cortes convenientes. Fica confirmada a observabilidade dos processos no LHC com menos de $20 \mathrm{fb}^{-1}$ de luminosidade acumulada, com o acelerador funcionando a energia máxima (14 TeV), sendo os principais resultados divulgados na referência [37].

Nota-se que os resultados obtidos com base na equação (5.15), seguindose a estratégia de cortes apresentada na seção 5.2, mostram que será possível confirmar eventos com significância superior a $5 \sigma$ para o processo $p p \rightarrow d_{4} \bar{b}$ com aproximadamente $15 \mathrm{fb}^{-1}$ de luminosidade acumulada, com o LHC funcionando a $14 \mathrm{TeV}$. Com o LHC a $7 \mathrm{TeV}$ não será possível tal confirmação, uma vez que a luminosidade acumulada nesta fase de operação não será suficiente para permitir a confirmação de eventos, considerando-se o fundo. Isto se deve ao fato de ser considerado apenas casos onde $m_{1}>2 \mathrm{TeV}$, consistentes com o modelo utilizado. Estão sob estudos casos mais gerais, onde não considera-se o modelo aqui utilizado, mas sim modelos que como este apresentam troca de sabor por correntes neutras, por meio de um octeto de cor. Nestes estudos, consideram-se casos onde $m_{o c} \geq 1 \mathrm{TeV}$ e poderiam resultar em eventos observáveis no LHC a $7 \mathrm{TeV}$. Tais estudos serão divulgados na referência [37].

Na seção 5.3 nota-se que será possível confirmar eventos também do processo $p p \rightarrow u_{4} \bar{t}$ com luminosidade acumulada de aproximadamente $42 \mathrm{fb}^{-1}$, a $14 \mathrm{TeV}$. Pode ser notado também que esta confirmação deve ser posterior, uma vez que a topologia deste processo é idêntica à do fundo, tornando mais difícil a aplicação de cortes.

A fenomenologia de modelos de quebra da simetria eletrofraca foi es- 
tudada, cumprindo-se também a tarefa de mostrar a viabilidade de observação experimental dos processos no LHC. Dada a diversidade de processos disponíveis a energias mais elevadas, faz-se necessário o estabelecimento de estratégias de observação e a cautelosa escolha de processos que sirvam como guia aos experimentalistas, com base em teorias e princípios físicos.

As técnicas utilizadas neste trabalho podem ser úteis no contexto de outros modelos com troca de sabor na produção de férmions pesados. Outros aspectos fenomenológicos desses modelos ficam ainda a serem estudados, como, por exemplo, o setor escalar composto, tanto trocando quanto conservando sabor. 


\section{Bibliografia}

[1] Amsler, C., et al., Review of Particle Physics, Physics Letters B, 667, 1, 2008 .

[2] Donoghue, J. F., Golowich, E., Holstein, B. R., Dynamics of the Standard Model, Cambridge, 1992.

[3] Bardeen, W. A., Hill, C. T., Lindner, M., Minimal Dynamical Symmetry Breaking of the Standard Model, Phys. Rev. D 41: 1647, 1990.

[4] Randall, L., Sundrum, R., A Large Mass Hierarchy from a Small Extra Dimension, Phys. Rev. Lett. 83: 3370-3373, 1999.

[5] Gherghetta, T., Pomarol, A., Bulk Fields and Supersymmetry in a Slice of AdS, Nucl. Phys. B 586: 141-162, 2000.

[6] Grossman, Y., Neubert, M., Neutrino Masses and Mixings in NonFactorizable Geometry, Phys. Lett. B 474: 361-371, 2000.

[7] Gherghetta, T., Les Houches Lectures on Warped Models and Holography, "Les Houches 2005, Particle Physics Beyond the Standard Model"263-311, 2005.

[8] Burdman, G., Da Rold, L., Electroweak Symmetry Breaking from a Holographic Fourth Generation, JHEP 0712: 086, 2007.

[9] Burdman, G., Da Rold, L., Éboli, O. J. P., Matheus, R. D., A Strongly Coupled Fourth Generation at the LHC, Phys. Rev. D 79: 075026, 2009.

[10] Para uma revisão vide, por exemplo: Sobol, I., O método de Monte Carlo, MIR, 1983. 
[11] Itzykson, C., Zuber, J.-B., Quantum Field Theory, Dover, 2005.

[12] Burdman, G., Theories with Extra Dimensions, AIP Conf. Proc. 753: 390-399, 2005.

[13] Gol'fand, Yu. A., Likhtman, E. P., Extension of the Algebra of Poincaré Group Generators and Violation of p Invariance, JETP Lett. 13: 323326, 1971.

[14] Volkov, D. V., Akulov, V. P., Is the Neutrino a Goldstone Particle?, Phys. Lett. B 46: 109-110, 1973.

[15] Wess, J., Zumino, B., Supergauge Transformations in Four-Dimensions, Nucl. Phys. B 70: 39-50, 1974.

[16] Wess, J., Zumino, B., A Lagrangian Model Invariant Under Supergauge Transformations, Phys. Lett. B 49: 52, 1974.

[17] Para uma revisão vide, por exemplo: Martin, S. P., A Supersymmetry Primer, "Kane, G.L. (ed.): Perspectives on supersymmetry II"1-153, 1997.

[18] Weinberg, S., Implications of Dynamical Symmetry Breaking, Physical Review D 13, 4: 974-996, 1976.

[19] Weinberg, S., Implications of Dynamical Symmetry Breaking: An Addendum, Physical Review D 19, 4: 1277-1280, 1979.

[20] Susskind, L., Dynamics of Spontaneous Symmetry Breaking in the Weinberg-Salam Theory, Physical Review D 20, 10: 2619-2625, 1979.

[21] Para uma revisão vide, por exemplo: Hill, C. T., Simmons, E. H., Strong Dynamics and Electroweak Symmetry Breaking, Phys. Rept. 381: 235402, 2003, Erratum-ibid. 390: 553-554, 2004.

[22] Dimopoulos, S., Susskind, L., Mass Without Scalars, Nuclear Physics B155, 1: 237-252, 1979.

[23] Eichten, E., Lane, K., Dynamical Breaking of Weak Interaction Symmetries, Physics Letters B 90 1-2: 125-130, 1980.

[24] Arkani-Hamed, N., Dimopoulos, S., Dvali, G. R., The Hierarchy Problem and New Dimensions at a Millimeter, Phys.Lett. B 429: 263-272, 1998. 
[25] Appelquist, T., Cheng, H.-C., Dobrescu, B. A., Bounds on universal extra dimensions, Phys. Rev. D 64: 035002, 2001.

[26] Para uma revisão vide, por exemplo: Pérez-Lorenzana, A., An Introduction to Extra Dimensions, J. Phys. Conf. Ser. 18: 224-269, 2005.

[27] Abramowitz, M., et al., Handbook of Mathematical Functions, Dover, 1965.

[28] Weinberg, S., Gravitation and Cosmology: Principles and Applications of the General Theory of Relativity, Wiley, 1972.

[29] Conway, J., et al., CDF Public Note, CDF/PUB/TOP/PUBLIC/10395, 2011.

Aaltonen, T., et al. (CDF Collaboration), Search for heavy bottom-like quarks decaying to an electron or muon and jets in $p \bar{p}$ collisions at $\sqrt{s}=1.96 \mathrm{Te} V$, arXiv:1101.5728v1 [hep-ex], 2011.

[30] Csaki, C., Falkowski, A., Weiler, A., A Simple Flavor Protection for RS, Phys. Rev. D 80, 016001, 2009.

[31] Santiago, J., Minimal Flavor Protection: A New Flavor Paradigm in Warped Models, JHEP 0812, 046, 2008.

[32] Peskin, M. E., Takeuchi, T., New Constraint on a Strongly Interacting Higgs Sector, Phys. Rev. Lett. 65, 964, 1990.

[33] Agashe, K., Delgado, A., May, M. J., Sundrum, R., RS1, Custodial Isospin and Precision Tests, JHEP 0308: 050, 2003.

[34] Cabrer, J. A., Gersdorff, G., Quiros, M., Warped Electroweak Breaking Without Custodial Symmetry, Phys. Lett. B 697: 208-214, 2011.

[35] Nambu, Y., Jona-Lasinio, G., Dynamical Model of Elementary Particles Based on an Analogy with Superconductivity, i, Phys. Rev., 122:345, 1961.

[36] Burdman, G., Da Rold, L., Matheus, R. D., The Lepton Sector of a Fourth Generation, Phys. Rev. D 82: 055015, 2010.

[37] Burdman, G., Lascio, E. R., em preparação. 
[38] Alwall, J., et al., MadGraph/MadEvent v4: The New Web Generation, JHEP 0709: 028, 2007.

[39] Meade, P., Reece, M., BRIDGE: Branching Ratio Inquiry/Decay Generated Events, arXiv:hep-ph/0703031v2, 2007. 